(C) 2014

AREZOO SADRINEZHAD

ALL RIGHTS RESERVED 


\title{
MULTIAXIAL PROBABILISTIC ELASTIC-PLASTIC CONSTITUTIVE SIMULATIONS OF SOILS
}

\author{
A Dissertation \\ Presented to \\ The Graduate Faculty of The University of Akron
}

\author{
In Partial Fulfillment \\ of the Requirements for the Degree \\ Doctor of Philosophy
}

Arezoo Sadrinezhad

August, 2014 


\title{
MULTIAXIAL PROBABILISTIC ELASTIC-PLASTIC CONSTITUTIVE
}

\section{SIMULATIONS OF SOILS}

\author{
Arezoo Sadrinezhad
}

Dissertation

Approved:

Accepted:

Advisor

Dr. Kallol Sett

Co-Advisor

Dr. S. I. Hariharan

Committee Member

Dr. Robert Y. Liang

Committee Member

Dr. David Roke

Committee Member

Dr. Wieslaw K. Binienda
Department Chair

Dr. Wieslaw K. Binienda

Dean of the College

Dr. George K. Haritos

Dean of the Graduate School

Dr. George R. Newkome

Date

Committee Member

Dr. Ali Hajjafar 


\begin{abstract}
Fokker-Planck-Kolmogorov (FPK) equation approach has recently been developed to simulate elastic-plastic constitutive behaviors of materials with uncertain material properties. The FPK equation approach transforms the stochastic constitutive rate equation, which is a stochastic, nonlinear, ordinary differential equation (ODE) in the stress-pseudo time space into a second-order accurate, deterministic, linear FPK partial differential equation (PDE) in the probability density of stress-pseudo time space. This approach does not suffer from the drawbacks of the traditional approaches such as the Monte Carlo approach and the perturbation approach for solving nonlinear ODEs with random coefficients.

In this study, the existing one dimensional FPK framework for probabilistic constitutive modeling of soils is extended to multi-dimension. However, the multivariate FPK PDEs cannot be solved using the traditional mathematical techniques such as finite difference techniques due to their high computational cost. Therefore, computationally efficient algorithms based on the Fourier spectral approach are developed for solving a class of FPK PDEs that arises in probabilistic elasto-plasticity. This class includes linear FPK PDEs in (stress) space and (pseudo) time - having space-independent but time-dependent, and both space- and time-dependent coef-


ficients - with impulse initial conditions and reflecting boundary conditions. The solution algorithms, rely on first mapping the stress space of the governing PDE between 0 and $2 \pi$ using the change of coordinates rule, followed by approximating the solution of the PDE in the $2 \pi$-periodic domain by a finite Fourier series in the stress space and unknown time-dependent solution coefficients. Finally, the timedependent solution coefficients are obtained from the initial condition. The accuracy and efficiency of the developed algorithms are tested.

The developed algorithms are used to simulate uniaxial and multiaxial, monotonic and cyclic constitutive behaviors of uncertain elasto-plastic soils. In particular, the effects of uncertainties in soil properties on constitutive behaviors of soils subjected to an unconfined compression test and to an unconsolidated undrained triaxial test are evaluated. To this end, the governing FPK equation of probabilistic elastoplasticity is specialized to uniaxial stress space and to two dimensional principal stress space. Then, the specialized FPK PDEs are solved using the developed algorithms. Simulation results show that by considering soil uncertainties in the constitutive simulation, more realistic constitutive behavior can be ontained which agrees well with the principles of micromechanics. It is also observed that by explicit treatment to soil uncertainties, some of the important aspects of soil behaviors-for example, hardening and Bauschinger effect- can be captured even with the simplest elastic-perfectly plastic von Mises (two-parameters) model. 


\section{ACKNOWLEDGEMENTS}

First and foremost, I would like to express my sincere appreciation and gratitude to my advisor, Dr. Kallol Sett, for his patience, guidance and enthusiastic encouragement throughout my researches at the University of Akron. Besides completing a $\mathrm{PhD}$ dissertation, he stimulated and directed me in publishing conference papers, journal papers and a book chapter. During these projects, he was always there to provide necessary assistance and academic resources. Without his support, I could not have completed my research.

Special thanks go to Dr. S. I. Hariharan, for agreeing to serve as my official advisor after Dr. Kallol Sett moved to the State University of New York at Buffalo. I thank Dr. S. I. Hariharan for helping me with the mathematical aspects of my dissertation. I also thank him for his guidance, encouragement and support during the last year of my $\mathrm{PhD}$. He was always there to listen and to give me sincere advice about anything I hoped for. Without his encouragements and continuous guidance, I could not have finished this dissertation.

I would also like to thank Dharma Teja Akkineni for helping me to verify my simulation results.

I am indebted to my committee members: Dr. Robert Y. Liang, Dr. David 
Roke, Dr. Wieslaw K. Binienda and Dr. Ali Hajjafar. They have provided, with kindness, their valuable comments and suggestions, which are precious to me.

Financial support by NASA/Ohio Space Grant Consortium, NSF (through award no. CMMI-1200196; cognizant program director: Dr. Richard Fragaszy) and the Department of Civil Engineering at the University of Akron is also greatly appreciated.

My gratitude is also extended to my family, for the continuous support and unconditional love, and constant prayers. I am forever grateful to them because they made me what I am and helped me achieve my goals.

I would also like to thank my friends, Ehsan Atefi, Amirhossein Molavi Tabrizi, Kow Okyere Eshun and Ali Sangghaleh for their help, advice, support and encouragement during my doctoral studies. I could not have completed my $\mathrm{PhD}$ without the support of these wonderful friends. 
TABLE OF CONTENTS

Page

LIST OF TABLES ..................... ix

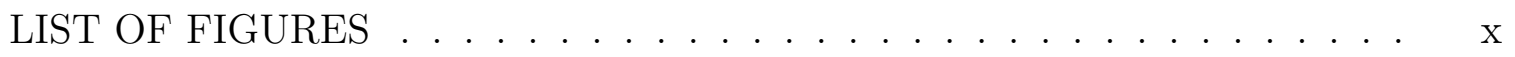

CHAPTER

I. MOTIVATION ....................... 1

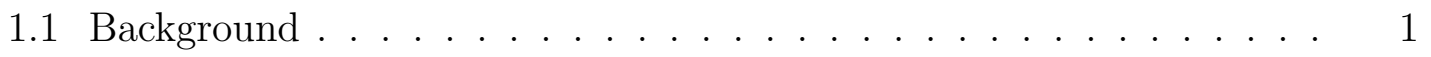

1.2 Scope of Study . . . . . . . . . . . . . . . . . . . . . 11

1.3 Summary of Contents . . . . . . . . . . . . . . . . 12

II. FOKKER-PLANCK-KOLMOGOROV EQUATION APPROACH TO PROBABILISTIC ELASTO-PLASTICITY . . . . . . . . . . 14

2.1 Mathematical Forms of the Governing FPK Equations . . . . . . . 14

III. SOLUTION ALGORITHMS FOR THE FOKKER-PLANCKKOLMOGOROV EQUATIONS IN PROBABILISTIC ELASTO-

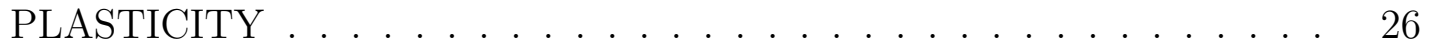

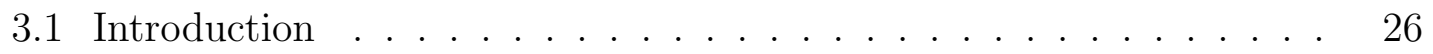

3.2 Finite Difference Techniques . . . . . . . . . . . . . . . . 28

3.3 Fourier Spectral Method . . . . . . . . . . . . . . . . . . 38

IV. SIMULATION OF UNCERTAIN CONSTITUTIVE BEHAVIORS OF

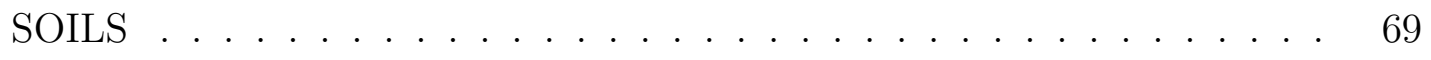

4.1 Simulation of Uncertain Monotonic Constitutive Behaviors of Soils . 69

4.2 Simulation of Uncertain Cyclic Constitutive Behaviors of Soils . . . 95 


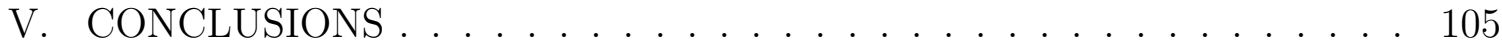

5.1 Summary and Conclusions . . . . . . . . . . . . . . 105

5.2 Future work . . . . . . . . . . . . . . . . . . 108

BIBLIOGRAPHY . . . . . . . . . . . . . . . . . . 110 


\section{LIST OF FIGURES}

Figure $\quad$ Page

1.1 Inherent soil variability (modified from Baecher and Christian [4]) . . . 1

1.2 Soil samples are obtained from a limited number of boreholes (modified from Baecher and Christian [4]) . . . . . . . . . . . . . 3

1.3 Testing uncertainty $[34] \ldots \ldots \ldots \ldots$. . . . . . . . . . . 4

1.4 Transformation relation uncertainty $[18] \ldots \ldots \ldots$

1.5 Stress-strain behavior of soils: (a) deterministic behavior (b) uncertain behavior $([24]) \ldots \ldots \ldots \ldots$. . . . . . . . . . . . . . 6

1.6 Stress domain discretization of Fokker-Planck-Kolmogorov PDE [44] . 11

3.1 Finite difference grid used for discretizing univariate FPK equation . $\quad 29$

3.2 Finite difference grid used for discretizing bivariate FPK equation . . . 32

3.3 Forward difference is used for the boundary at the left side . . . . . . . 33

3.4 Spectral methods versus finite difference methods (modified from J. P. Boyd [5]) . . . . . . . . . . . . . . . . . . . . . . . . 40

3.5 Mapping the stress space between 0 and $2 \pi$ using the change of coordinates rule . . . . . . . . . . . . . . . . . 43

4.1 Probability density function (PDF) of the axial stress corresponding to an axial strain of $1 \%$ for an uncertain linear elastic soil under an unconfined compression test. . . . . . . . . . . . . . . . . 71

4.2 Simulated probabilistic elastic behavior in terms evolutionary marginal mean and marginal mean \pm standard deviation behaviors. . . 74 
4.3 Joint probability density function (PDF) of the axial stress $\left(\sigma_{1}\right)$ and radial stress $\left(\sigma_{2}\right)$ at an axial strain of $1 \%$ for an uncertain elastic soil under an unconsolidated undrained triaxial compression test. . . . 76

4.4 Simulated probabilistic undrained elastic behavior in terms of (a) deviator stress, $q$ versus axial strain, $\epsilon_{a}$, and (b) deviator stress, $q$ versus triaxial shear strain, $\epsilon_{q} \ldots \ldots \ldots \ldots \ldots$

4.5 Simulated probabilistic undrained elastic behavior in terms of pore pressure, $u$ versus triaxial shear strain, $\epsilon_{q} \ldots \ldots$. . . . . . . 81

4.6 Probability density function (PDF) of the axial stress corresponding to an axial strain of $1 \%$ for an uncertain von Mises elastic-perfectly plastic soil under an unconfined compression test. . . . . . . . . 83

4.7 Simulated probabilistic elastic-perfectly plastic behavior in terms of the evolutionary mean and mean \pm standard deviation behaviors. . . . 84

4.8 Effect of the number of Fourier terms on the Probability density function (PDF) of the axial stress corresponding to an axial strain of $1 \%$ for an uncertain von Mises elastic-perfectly plastic soil under an unconfined compression test. . . . . . . . . . . . .

4.9 Effect of the number of Fourier terms on the simulated evolutionary mean and standard deviation behaviors of the axial stress with the axial strain for an uncertain von Mises elastic-perfectly plastic soil under an unconfined compression test. . . . . . . . . . . 86

4.10 Joint probability density function (PDF) of the axial stress $\left(\sigma_{1}\right)$ and radial stress $\left(\sigma_{2}\right)$ at an axial strain of $1 \%$ for an uncertain von Mises elastic-perfectly plastic soil under an unconsolidated undrained triaxial compression test. . . . . . . . . . . . . . . 88

4.11 Probability density function (PDF) of the (a) $\sigma_{1}$ corresponding to an axial strain of $1 \%$ and (b) $\sigma_{2}$ corresponding to an axial strain of $1 \%$.

4.12 Simulated probabilistic undrained elastic-perfectly plastic behavior in terms of the (a) deviator stress, $q$ versus axial strain, $\epsilon_{a}$ and (b) deviator stress, $q$ versus triaxial shear strain, $\epsilon_{q} \ldots \ldots \ldots 91$

4.13 Simulated probabilistic undrained elastic-perfectly plastic behavior in terms of the pore pressure, $u$ versus triaxial shear strain, $\epsilon_{q} \ldots \ldots 92$ 
4.14 Effect of the number of Fourier terms on the simulated evolutionary marginal mean and marginal standard deviation of the axial stress for an uncertain von Mises elastic-perfectly plastic soil under an unconsolidated undrained triaxial compression test. . . . . . . . . . .

4.15 Effect of the number of Fourier terms on the simulated evolutionary marginal mean and marginal standard deviation of the radial stress for an uncertain von Mises elastic-perfectly plastic soil under an unconsolidated undrained triaxial compression test. . . . . . . . . .

4.16 Effect of the number of Fourier terms on the simulated evolutionary covariance between the axial stress and radial stress with the axial strain for an uncertain von Mises elastic-perfectly plastic soil under an unconsolidated undrained triaxial compression test. . . . . . . . . . 94

4.17 Effect of the number of Fourier terms on the simulated evolutionary correlation coefficient of the axial stress and radial stress with the axial strain for an uncertain von Mises elastic-perfectly plastic soil under an unconsolidated undrained triaxial compression test. . . . . . . 95

4.18 Probability density function (PDF) of the stress for an uncertain von Mises cyclic elastic-perfectly plastic soil under an unconfined compression test. . . . . . . . . . . . . . . .

4.19 Simulated probabilistic cyclic elastic-perfectly plastic behavior in terms of the evolutionary mean behavior. . . . . . . . . . . . .

4.20 Simulated probabilistic cyclic elastic-perfectly plastic behavior in terms of the evolutionary standard deviation behavior. . . . . . . . .

4.21 Probability density function (PDF) of the axial stress for an uncertain von Mises cyclic elastic-perfectly plastic soil under an unconfined compression test. . . . . . . . . . . . . . . . . . 102

4.22 Probability density function (PDF) of the radial stress for an uncertain von Mises cyclic elastic-perfectly plastic soil under an unconfined compression test. . . . . . . . . . . . . . . . . . . 102

4.23 Simulated probabilistic cyclic elastic-perfectly plastic behavior in terms of the evolutionary mean of deviatoric stress with axial strain. . . 103

4.24 Simulated probabilistic cyclic elastic-perfectly plastic behavior in terms of the evolutionary standard deviation of deviatoric stress with axial strain. . . . . . . . . . . . . . . . . . . . . . . . . 104 
4.25 Simulated probabilistic cyclic elastic-perfectly plastic behavior in terms of the evolutionary mean of deviatoric stress with evolutionary mean effective stress. . . . . . . . . . . . . . . . . . . . 104 


\section{CHAPTER I}

\section{MOTIVATION}

\subsection{Background}

Simulation of soil constitutive behavior is inherently uncertain. This is due to the uncertainty in soil properties. Soils are nature provided materials, and they form by weathering processes during thousands of years. Therefore, a soil deposit consists of different layers. Depending on natural geologic processes that form the soil deposit, the soil properties may vary from one layer to another or even within a layer (Figure 1.1).

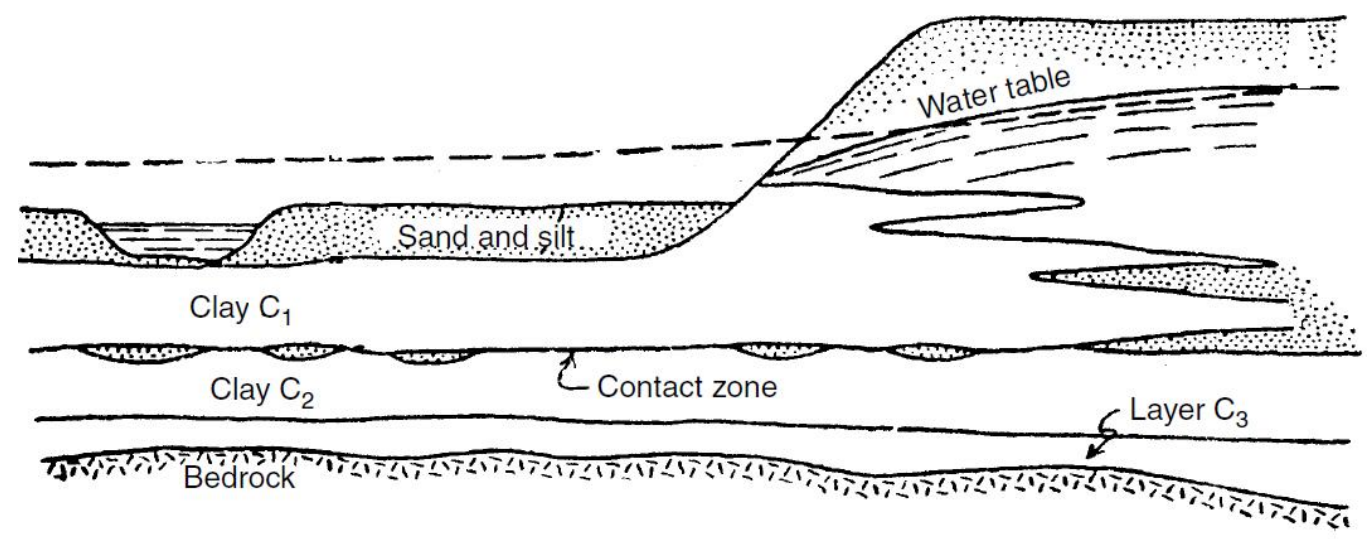

Figure 1.1: Inherent soil variability (modified from Baecher and Christian [4]) 
However, the traditional deterministic approach to soil constitutive simulation refrains from explicit treatment of these uncertainties in soil properties and instead deals with them using ad hoc factors of safety. This approach not only results in over predictions, but also in some cases - depending upon the degree of nonlinearity of the models - even in unsafe predictions. Therefore, in recent years, with more and more emphasis on reliability, the geotechnical engineering community is increasingly agreeing that any predictions must include quantifiable measures of uncertainty. In general, the uncertainties of soil properties are attributed to three primary sources:

1. The inherent variability of soil deposits - Generally, a soil deposit consists of different layers. Depending on the soil forming process, the soil properties may vary from one layer to another or even within a layer (Figures 1.1). If one could obtain the soil properties at each point, there would be nothing random. However, this is not practical due to time and money concerns. In the real world, soil properties are usually obtained at specific borehole locations (Figure 1.2), and the soil properties at the intermediate locations should be statistically predicted. This process introduces uncertainties in the soil properties [12, 13, 11].

2. Measurement uncertainties - They result from imperfection of instruments, error in registering quantities, and human error of test-operators [17, 38, 33, 30]. Figure 1.3 shows the values of undrained shear strength, one of the most important properties of the soil, measured from three different field tests (CPT, 


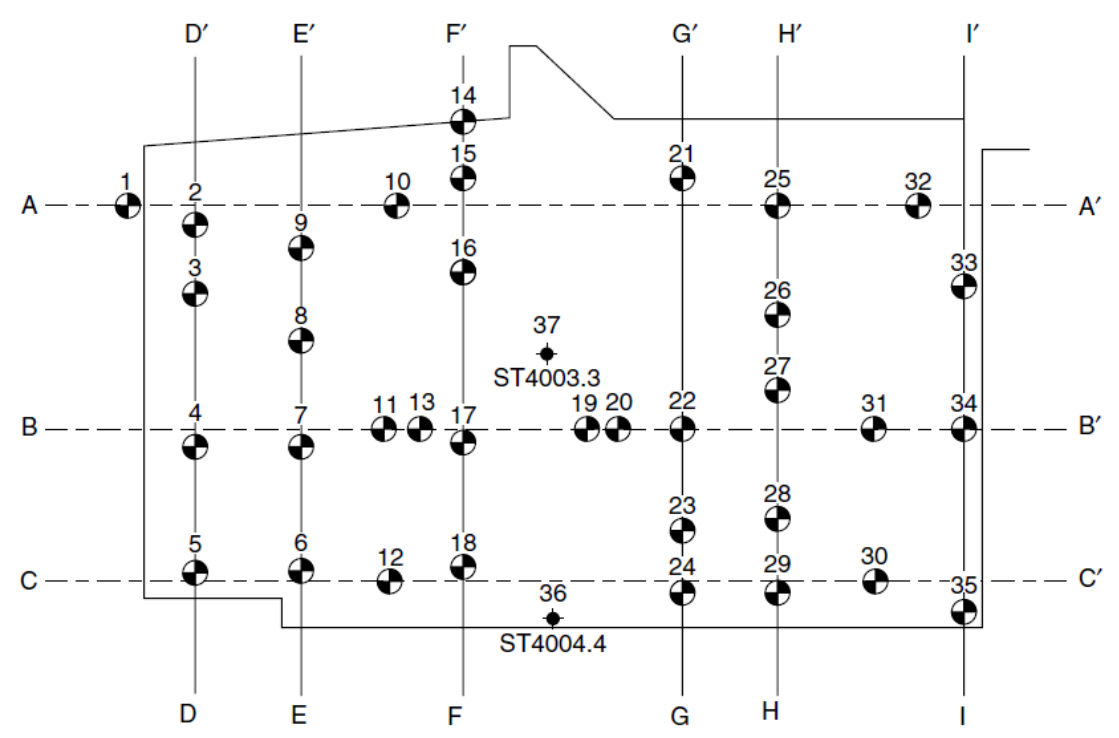

Figure 1.2: Soil samples are obtained from a limited number of boreholes (modified from Baecher and Christian [4])

SPT, and DMT), and one laboratory test (triaxial test). As can be seen in this figure, the value of undrained shear strength at a specific depth is different from one test to another.

3. Transformation relation uncertainty - Some of the soil properties may not be obtained directly from the commonly used field tests e.g., standard penetration test, cone penetration test, etc. Transformation relation uncertainty is introduced when empirical or other correlation models are used to compute soil properties from field measurements [39]. For instance, undrained shear strength $\left(S_{u}\right)$ can be estimated indirectly from SPT N values, the sum of the number of blows required for the second and third $6 \mathrm{in}$ of penetration, obtained from the SPT test (Figure 1.4). 


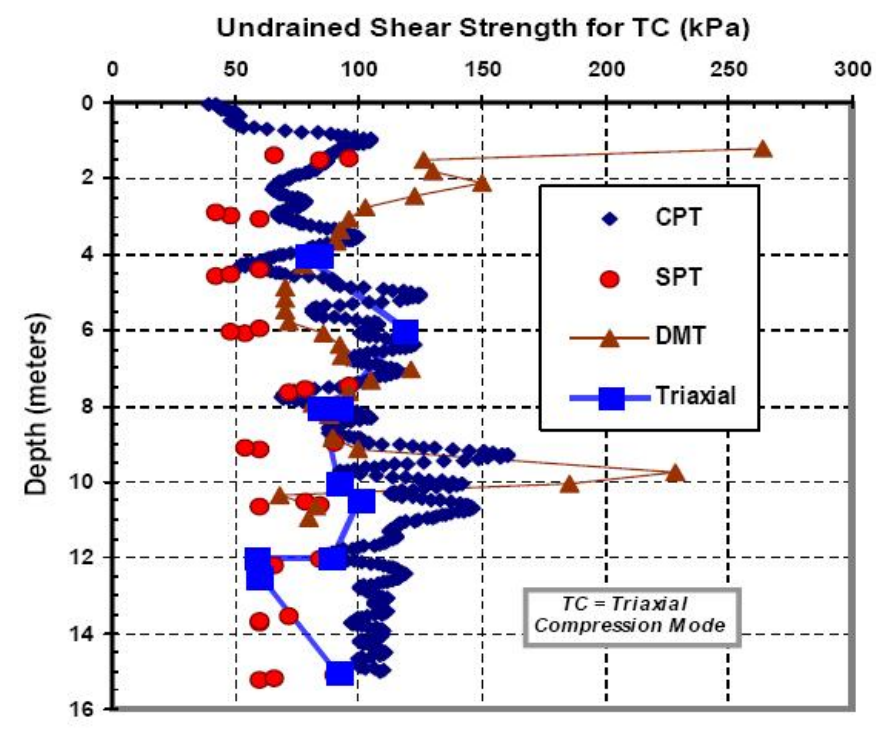

Figure 1.3: Testing uncertainty [34]

Due to these sources of uncertainty, the variabilities of soil properties are generally quite large. Figures 1.5 (a)-(b) compare the deterministic and uncertain elasto-plastic behavior of soils. As can be seen in Figure 1.5 (b), if soil properties such as elastic modulus, yield strength, and hardening parameters become uncertain, the behavior of the same soils could be very different. Therefore, in order to provide a rational and realistic constitutive soil behavior, the uncertainty in soil properties should be quantified and accounted for in our simulations.

The most common method to quantify the uncertainty in material properties is the random field approach. To this end, the in-situ and lab test data should be transformed to the required soil properties using transformation relations first. For example, elastic modulus and undrained shear strength, the two required soil param- 


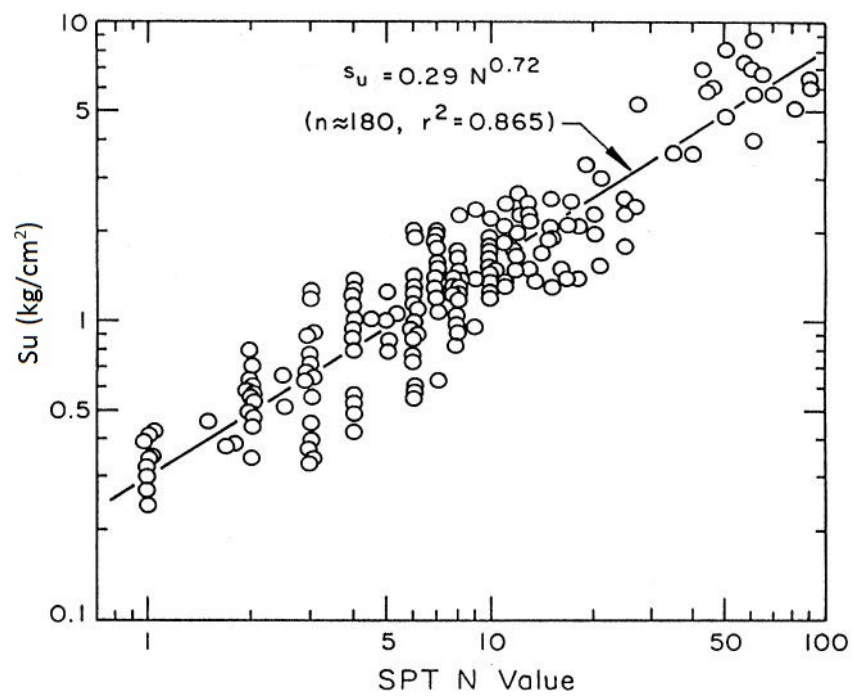

Figure 1.4: Transformation relation uncertainty [18]

eters for constitutive modeling of soils, can be obtained from the cone penetration test's (CPT) data using Eq. (1.3) and Eq. (1.4), respectively.

$$
\begin{gathered}
V_{s}=1.75 q_{t}^{0.627} \\
G=\frac{\gamma}{g} V_{s}^{2} \\
E=2 G(1+\nu)
\end{gathered}
$$

where $V_{s}, G, \gamma, E$ and $\nu$ are shear wave velocity, shear modulus, unit weight, acceleration due to gravity, elastic modulus, and Poisson's ratio, respectively. Undrained shear strength can be obtained as:

$$
c_{u}=\frac{\left(q_{t}-\sigma_{v 0}\right)}{N_{k t}}
$$

where $q_{t}, \sigma_{v 0}$ and $N_{k t}$ are the cone tip resistance, vertical overburden pressure, and bearing capacity factor, respectively. 


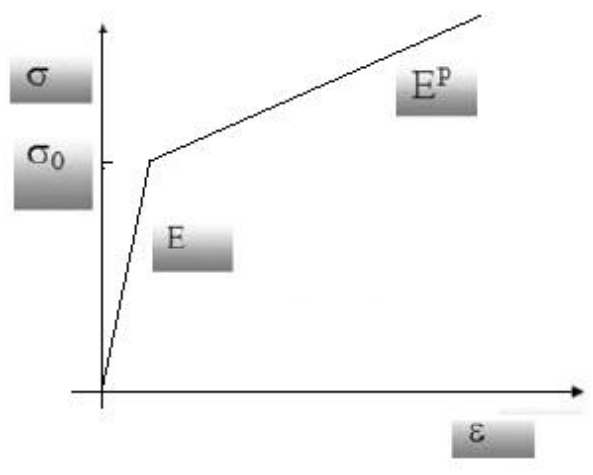

(a)

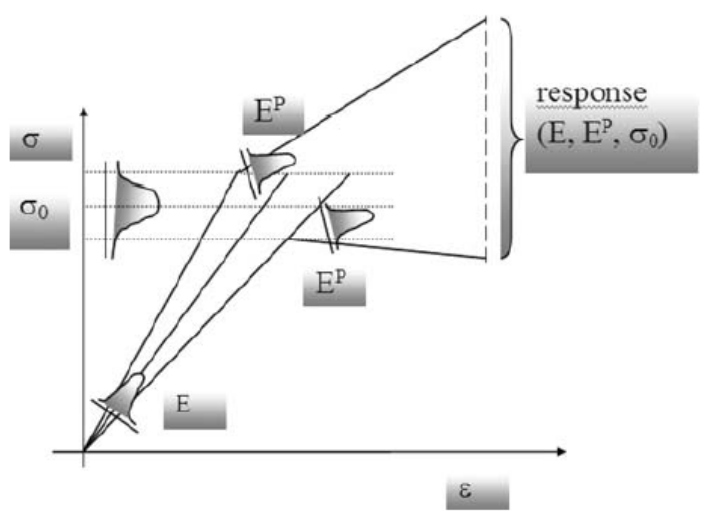

(b)

Figure 1.5: Stress-strain behavior of soils: (a) deterministic behavior (b) uncertain behavior $([24])$

Then, every soil property should be modeled as a random field by a set of correlated random variables. It should be mentioned that a (scalar) random variable is a function $Z$ assigning to an event $\omega \in \Omega$ (an outcome in a sample space), a number $z$ on a real line:

$$
Z: \Omega \rightarrow R, Z(\omega)=z
$$

In modeling a soil property as a random field, the measured soil property at each borehole location becomes a realization of that random field. Based on a number of realizations (i.e., borehole measurements), the parameters of the random field soil property, such as autocovariance function and the correlation length, can be evaluated. Note that the autocovariance of the random field is the covariance of two random variables $Z\left(x_{1}\right)$ and $Z\left(x_{2}\right)$, and the correlation length is defined as the area under the correlation function [12]. 
Those random fields will appear in the governing equations of mechanics which should be solved to simulate the static and dynamic behaviors of elasto-plastic geo-solids and geo-structures. These equations are equilibrium equation (Eq. (1.6)), strain-displacement relations (Eq. (1.7)), and constitutive equation (Eq. (1.8)). The solution to these equations may be found using analytical or numerical approaches. However, the analytical solution to these equations does not typically exist. Therefore, the numerical approaches such as the stochastic finite element method (SFEM) should be employed for solving these equations.

$$
\begin{gathered}
\frac{\partial \sigma_{i j}}{\partial x_{j}}+b_{i}=\rho \ddot{u}_{i} \\
\epsilon_{i j}=\frac{1}{2}\left(\frac{\partial u_{i}}{\partial x_{j}}+\frac{\partial u_{j}}{\partial x_{i}}\right) \\
\frac{d \sigma_{i j}}{d t}=C_{i j k l} \frac{d \epsilon_{k l}}{d t}
\end{gathered}
$$

In stochastic finite element setting, the random field material properties should be discretized into finite numbers of independent basic random variables. This discretization can be done using different techniques such as Karhunen-Loève (KL) expansion $[26,32,15,46]$, Polynomial chaos (PL) expansion $[49,15,46]$ and shape function expansion $[15,52,46]$. The obtained random variables appear in the constitutive rate equation (operator $C$ in Eq. (1.8)) which makes this equation a stochastic differential equation.

One way to solve stochastic differential equations is the Monte Carlo simulation (MCS) technique which builds statistics of the response using a large ensemble of realizations obtained by sampling the random variables or random processes 
[37, 40, 10, 29, 16]. MCS technique, in general, is very straightforward and can be used to probabilistically simulate any problem whose deterministic solution - either analytical or numerical - is known. Monte Carlo simulation involves three steps [35]:

1. Generating $\mathrm{N}$ samples of the random variables.

2. Performing the deterministic analysis for each random variable generated in the first step.

3. Collecting the deterministic solutions and analyzing them statistically to estimate properties of the solution of the stochastic problem.

However, the accuracy of the MCS technique depends upon the number of realizations $(N)$ used in the simulation process; the Monte Carlo estimates approach their true values as the number of deterministic runs, $N$ approaches infinity. Because of the above, accurate probabilistic simulation using the MCS approach becomes extremely computational-intensive especially for non-linear problems with multiple uncertain material properties. For example, for the 3-D elasto-plastic model with two random variable material parameters (e.g., Young's modulus $(E)$ and undrained shear strength $\left(S_{u}\right)$ for simplest von Mises model with no hardening), one needs at least $10,000^{2}=10^{8}$ runs of the computationally expensive deterministic model. In addition to this, the post-processing of the high volume of data is another problem with the Monte Carlo technique. To reduce the computational cost, a smaller number of statistically appropriate realizations can be used, however, this increases the error in the Monte Carlo simulations. Moreover, in a Monte Carlo setting, a probabilistic 
elastic-plastic problem is also difficult to parallelize due to necessary runtime interprocessor communications [42]. Due to this high computational cost in analysis and data processing, the Monte Carlo approach is impractical for large-scale problems, like an elasto-plastic dynamic problem in a finite element setting.

To overcome the drawbacks of the Monte Carlo technique, other methods such as the stochastic differential equation approach [14] or the stochastic finite element method $[28,15]$ have been used recently. However, so far, these methods are mostly formulated for linear elastic problems. The first attempt to propagate uncertainty in material properties through the elasto-plastic constitutive equations was made by Anders and Hori [1]. They employed the perturbation method to propagate uncertainties in modulus through the von Mises elastic-perfectly plastic material model $[1,2]$. The perturbation method is applicable to non-linear problems. However, this approach uses Taylor series expansion which restricts the use of this method to problems with small coefficients of variations (COVs). Comparisons with Monte Carlo simulations showed that the relatively accurate results can be obtained for COVs up to $20 \%$ [47]. Therefore, the perturbation approach may not be very accurate for simulation of behavior of soils because their COVs are rarely below 20\%. Moreover, perturbation method suffers from another problem called 'closure problem' which means in order to calculate lower-order moments, the information on higher-order moments is required [51].

Recently, in circumventing the above drawbacks of the Monte Carlo technique and the perturbation method, Jeremić et al [24] proposed a method for probabilistic 
elasto-plastic constitutive simulations. This approach utilizes a generic solution proposed by Kavvas [27] to stochastic differential equations with random coefficients and random forcing functions, and rewrites the general form of the elasto-plastic constitutive rate equation in the probability density space in obtaining an FPK equation which describes the evolution of the probability density function (PDF) of stress with pseudo time. Specialization of the FPK equation to any particular elastic-plastic constitutive model, followed by the solution of the specialized FPK equation provides evolution of the PDF of stress with strain following that constitutive model. Note that this approach provides the probabilistic constitutive response in a single run and thus reduces the high computational cost associated with conventional MCS technique. Moreover, it overcomes the closure problem [51] and 'small variance requirement' [47] associated with the perturbation approach.

The FPK approach to probabilistic elasto-plasticity has been used to simulate both monotonic and cyclic, hardening and softening type uncertain material behaviors $[45,43]$. It has also been successfully integrated with the spectral approach of the stochastic finite element method (SFEM) $[28,15]$ to simulate the stochastic elasticplastic boundary value problems in mechanics [46]. It is important to note that in the previous studies, which were only in 1-dimensional space, the finite difference technique was used to solve the FPK partial differential equation [44]. The FPK equation was semi-discretized in stress domain by the finite difference technique to obtain a set of linear simultaneous ODE systems (Figure 1.6). This set of linear simultaneous ODEs was solved using the central difference technique. Note, however, 


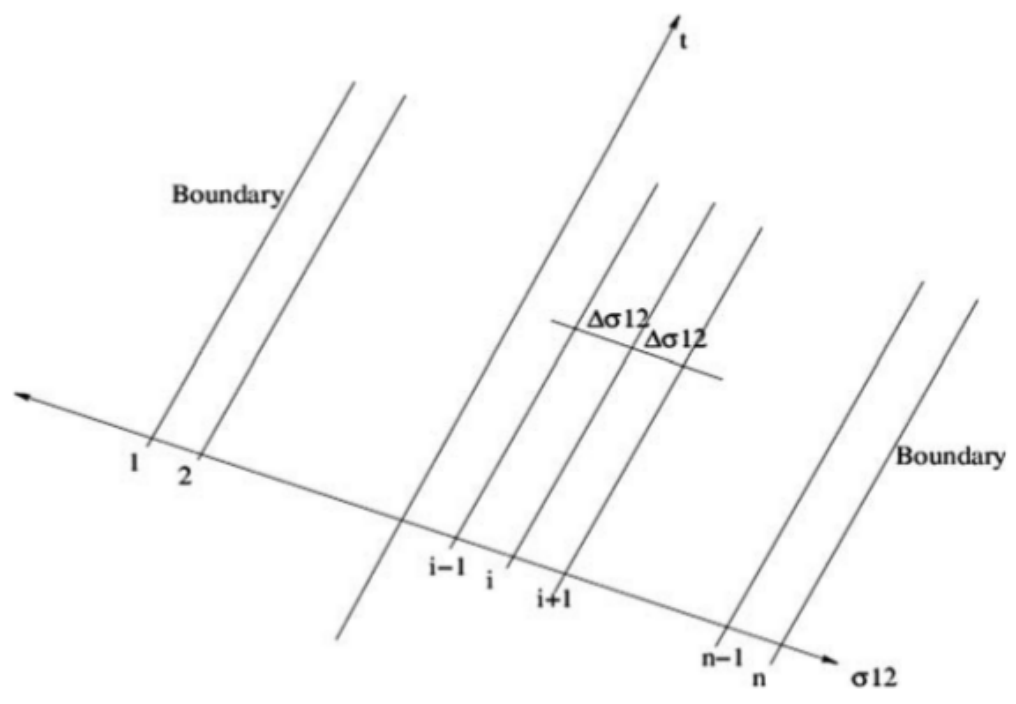

Figure 1.6: Stress domain discretization of Fokker-Planck-Kolmogorov PDE [44]

that for a multivariate FPK equation, the finite difference technique, which needs discretization in each direction, becomes computationally very expensive especially if performed at each Gauss point repeatedly in a stochastic finite element framework.

\subsection{Scope of Study}

The main goal of this research is to extend the existing one dimensional FPK framework for probabilistic constitutive modeling of soils to multi-dimension. More specifically, the objectives are:

1. Develop computationally efficient algorithms, based on the Fourier spectral approach, for solving univariate and multivariate FPK PDEs, with space- 
independent but time-dependent, and both space- and time-dependent coefficients, that commonly arise in probabilistic elasto-plasticity.

2. Test the accuracy and efficiency of the developed algorithms.

3. Use the developed algorithms to understand uniaxial and multiaxial, monotonic and cyclic constitutive behaviors of uncertain elasto-plastic soils. In particular, evaluate the effect of uncertainties in soil properties on constitutive behaviors of soils subjected to an unconfined compression test (a uniaxial problem) and to an unconsolidated undrained triaxial test (a multiaxial problem).

\subsection{Summary of Contents}

This dissertation is organized into six chapters. The first chapter provides the background and motivation of the research. The aim and outline of the dissertation are also included.

Chapter 2 presents the governing FPK equation of probabilistic elasto-plasticity in the most general forms. It also specializes the generalized equation to uniaxial stress space and to two dimensional principal stress space in probabilistically simulating stress-strain behaviors of clayey soils during the unconfined compression test and the unconsolidated undrained triaxial test, respectively. These specializations are demonstrated using both linear elastic constitutive model and von Mises elasticperfectly plastic model. 
Chapter 3 develops algorithms to solve the specialized FPK equations. First, an algorithm based on the finite difference method is presented. Then a set of computationally efficient algorithms based on the Fourier spectral method are proposed.

Chapter 4 presents the simulation results in terms of probabilistic monotonic and cyclic constitutive behaviors of clayey soils under the unconfined compression test and the unconsolidated undrained triaxial compression test. The accuracy and efficiency of the developed algorithms are also tested.

Conclusions are drawn in the last chapter. It also lists future research directions. 


\section{CHAPTER II}

\section{FOKKER-PLANCK-KOLMOGOROV EQUATION APPROACH TO PROBABILISTIC ELASTO-PLASTICITY}

In this chapter, first, the most general forms of the FPK equations in probabilistic elasto-plasticty are presented. Then, the general forms of the equations are specialized to uniaxial stress space and to two-dimensional principal stress space in order to simulate the two common laboratory constitutive experiments - unconfined compression test and unconsolidated undrained triaxial compression test -in geotechnical engineering. Note that, both elastic and von Mises elastic-perfectly plastic models are considered.

\subsection{Mathematical Forms of the Governing FPK Equations}

The most general, rate form of the constitutive equation for an uncertain, elasticplastic material, when written in the probability density space, becomes two coupled FPK PDEs of the following forms [24, 44, 45, 23, 43]:

$$
\begin{aligned}
\frac{\partial P(\boldsymbol{\sigma}, t)}{\partial t}= & -\frac{\partial}{\partial \sigma_{m n}}\left[N_{(1)_{m n}^{\sigma^{e q}}}(\boldsymbol{\sigma}, \boldsymbol{q} ; t) P(\boldsymbol{\sigma}, t)\right] \\
& +\frac{\partial^{2}}{\partial \sigma_{m n} \partial \sigma_{a b}}\left[N_{(2)_{m n a b} \sigma^{e q}}(\boldsymbol{\sigma}, \boldsymbol{q} ; t) P(\boldsymbol{\sigma}, t)\right]
\end{aligned}
$$




$$
\begin{aligned}
\frac{\partial \widetilde{P}(\boldsymbol{q}, t)}{\partial t}= & -\frac{\partial}{\partial q_{i}}\left[N_{(1)_{i}}^{I V^{e q}}(\boldsymbol{\sigma}, \boldsymbol{q} ; t) \widetilde{P}(\boldsymbol{q}, t)\right] \\
& +\frac{\partial^{2}}{\partial q_{i} \partial q_{j}}\left[N_{(2)_{i j}}^{I V^{e q}}(\boldsymbol{\sigma}, \boldsymbol{q} ; t) \widetilde{P}(\boldsymbol{q}, t)\right]
\end{aligned}
$$

where $P$ is the joint probability density of the components of the stress tensor, $\boldsymbol{\sigma}=$ $\left\{\sigma_{11}, \sigma_{22}, \sigma_{33}, \sigma_{12}, \sigma_{23}, \sigma_{31}\right\}, \widetilde{P}$ is the joint probability density of the internal variables, $\boldsymbol{q}=\left\{q_{1}, q_{2}, \cdots, q_{k}\right\}, t$ is the pseudo time of the constitutive rate equation, $N_{(1)_{m n}}^{\sigma^{e q}}$ and $N_{(2)_{\text {mnab }}}^{\sigma^{e q}}$ are the equivalent advection and diffusion coefficients, respectively, of the stress FPK PDE, and $N_{(1)_{i}}^{I V^{e q}}$ and $N_{(2)_{i j}}^{I V^{e q}}$ are the equivalent advection and diffusion coefficients, respectively, of the internal variable FPK PDE. Note that the indices, $m, n, a$, and $b$, appearing in Eq. (2.1) vary from 1 to 3 and that the indices, $i$ and $j$, appearing in Eq. (2.2) vary from 1 to $k$, where $k$ is the number of internal variables. Further note that the internal variable FPK PDE (Eq. (2.2)) may not always exist - e.g., for an elastic constitutive equation (the internal variables do not exist) or for an elastic-perfectly plastic constitutive equation (the internal variables do not evolve). For those cases, the probabilistic constitutive behavior of any material is fully described by the stress FPK PDE (Eq. (2.1)) alone.

The equivalent advection and diffusion coefficients of the FKP PDEs, $N_{(1)_{m n}}^{\sigma^{e q}}$, $N_{(2)_{\text {mnab }}}^{\sigma^{e q}}, N_{(1)_{i}}^{I V^{e q}}$, and $N_{(2)_{i j}}^{I e^{e q}}$, depend upon the probabilities that the stresses are inside or on the yield surface and are functions of the statistics of the material parameters and the rate of strain. The mathematical forms of the coefficients vary depending upon the type of constitutive model. For models based on the classical plasticity 
theory, they may be expressed in the following general forms:

$$
\begin{aligned}
& N_{(1)_{m n}}^{\sigma^{e q}}(\boldsymbol{\sigma}, \boldsymbol{q} ; t)=\operatorname{Pr}[F<0](\boldsymbol{\sigma}, \boldsymbol{q}) N_{(1)_{m n}}^{\sigma^{e l}} \\
& +\{1-\operatorname{Pr}[F<0](\boldsymbol{\sigma}, \boldsymbol{q})\} \quad N_{(1)_{m n}}^{\sigma^{p l}}(\boldsymbol{\sigma}, \boldsymbol{q} ; t) \\
& N_{(2)_{\text {mnab }}^{\sigma_{\text {ma }}}}^{\sigma^{e q}}(\boldsymbol{\sigma}, \boldsymbol{q} ; t)=\operatorname{Pr}[F<0](\boldsymbol{\sigma}, \boldsymbol{q}) N_{(2)_{\text {mnab }}^{\sigma^{e l}}}(t) \\
& +\{1-\operatorname{Pr}[F<0](\boldsymbol{\sigma}, \boldsymbol{q})\} \quad N_{(2)_{\text {mnab }}}^{\sigma^{p l}}(\boldsymbol{\sigma}, \boldsymbol{q} ; t) \\
& N_{(1)_{i}}^{I V^{e q}}(\boldsymbol{\sigma}, \boldsymbol{q} ; t)=\{1-\operatorname{Pr}[F<0](\boldsymbol{\sigma}, \boldsymbol{q})\} \quad N_{(1)_{i}}^{I V^{p l}}(\boldsymbol{\sigma}, \boldsymbol{q} ; t) \\
& N_{(2)_{i j}}^{I V^{e q}}(\boldsymbol{\sigma}, \boldsymbol{q} ; t)=\{1-\operatorname{Pr}[F<0](\boldsymbol{\sigma}, \boldsymbol{q})\} \quad N_{(2)_{i j}}^{I V^{p l}}(\boldsymbol{\sigma}, \boldsymbol{q} ; t)
\end{aligned}
$$

where $F$ is the yield function which is a function of $\boldsymbol{\sigma}$ and $\boldsymbol{q}, \operatorname{Pr}[F<0]$ is the probability that $F$ is less than zero, $N_{(1)_{m n}}^{\sigma^{e l}}$ and $N_{(2)_{m n a b}}^{\sigma^{e l}}$ are the advection and diffusion coefficients of the stress FPK PDE when the stresses are inside the yield surface (elastic state), $N_{(1)_{m n}}^{\sigma^{p l}}$ and $N_{(2)_{m n a b}}^{\sigma^{p l}}$ are the advection and diffusion coefficients of the stress FPK PDE when the stresses are on the yield surface (plastic state), and $N_{(1)_{i}}^{I V^{p l}}$ and $N_{(2)_{i j}}^{I V^{p l}}$ are the advection and diffusion coefficients of the internal variable FPK PDE when the stresses are on the yield surface. Note that the internal variables do not evolve when the stresses are inside the yield surface and hence there are no contributions of the elastic state on $N_{(1)_{i}}^{I V^{e q}}$ and $N_{(2)_{i j}}^{I V^{e q}}$ in Eqs. (2.5) and (2.6).

The elastic state advection and diffusion coefficients of the stress FPK PDE are functions of the statistics of the elastic modulus $\left(D_{m n r s}^{e l}\right)$ and the rate of strain $\left(\dot{\epsilon}_{r s}\right)$, while the plastic state advection and diffusion coefficients of the same are functions of the statistics of the plastic modulus $\left(D_{m n r s}^{p l}\right)$ and the rate of strain. They 
may be mathematically expressed in the following forms:

$$
\begin{aligned}
& N_{(1)_{m n}}^{\sigma^{e l}}=\left\langle D_{m n r s}^{e l} \dot{\epsilon}_{r s}\right\rangle \\
& N_{(2)_{m n a b}}^{\sigma^{e l}}(t)=t \operatorname{Cov}\left[D_{m n r s}^{e l} \dot{\epsilon}_{r s} ; D_{a b c d}^{e l} \dot{\epsilon}_{c d}\right] \\
& N_{(1)_{m n}}^{\sigma^{p l}}(\boldsymbol{\sigma}, \boldsymbol{q} ; t)=\left\langle D_{m n r s}^{p l}(\boldsymbol{\sigma}, \boldsymbol{q} ; t) \dot{\epsilon}_{r s}\right\rangle+ \\
& \int_{0}^{t} d \tau \operatorname{Cov}_{0}\left[\frac{\partial}{\partial \sigma_{a b}}\left\{D_{m n r s}^{p l}(\boldsymbol{\sigma}, \boldsymbol{q} ; t) \dot{\epsilon}_{r s}\right\} ; D_{a b c d}^{p l}(\boldsymbol{\sigma}, \boldsymbol{q} ; t-\tau) \dot{\epsilon}_{c d}\right] \\
& N_{(2)_{m n a b}^{\sigma^{p l}}}(\boldsymbol{\sigma}, \boldsymbol{q} ; t)= \\
& \int_{0}^{t} d \tau \operatorname{Cov}_{0}\left[D_{m n r s}^{p l}(\boldsymbol{\sigma}, \boldsymbol{q} ; t) \dot{\epsilon}_{r s} ; D_{a b c d}^{p l}(\boldsymbol{\sigma}, \boldsymbol{q} ; t-\tau) \dot{\epsilon}_{c d}\right]
\end{aligned}
$$

where $\langle\cdot\rangle$ denotes expectation operation, $\operatorname{Cov}[\cdot]$ denotes covariance operation, $\operatorname{Cov}_{0}[\cdot]$ denotes time-ordered covariance operation, and $\tau$ is the lag (pseudo) time. The elastic and plastic moduli, $D_{i j k l}^{e l}$ and $D_{i j k l}^{p l}$, assuming material isotropy, may be expressed in terms of Young's modulus $(E)$, Poisson's ratio $(\nu)$, yield function $(F)$, plastic potential function $(U)$, internal variables $\left(q_{n}\right)$, and rates of evolution of internal variables $\left(r_{n}\right)$ as $[6]$ :

$$
\begin{gathered}
D_{i j k l}^{e l}=\frac{E}{2(1+\nu)}\left[\frac{2 \nu}{1-2 \nu} \delta_{i j} \delta_{k l}+\delta_{i k} \delta_{j l}+\delta_{i l} \delta_{j k}\right] \\
D_{i j k l}^{p l}(\boldsymbol{\sigma}, \boldsymbol{q} ; t)=D_{i j k l}^{e l}-\frac{\frac{\partial F}{\partial \sigma_{o t}} D_{i j o t}^{e l} D_{p q k l}^{e l} \frac{\partial U}{\partial \sigma_{p q}}}{\frac{\partial U}{\partial \sigma_{a b}} D_{a b c d}^{e l} \frac{\partial F}{\partial \sigma_{c d}}-\frac{\partial F}{\partial q_{n}} r_{n}}
\end{gathered}
$$

where $\delta_{i j}$ is the Kronecker delta.

The (plastic state) advection and diffusion coefficients of the internal variable FPK PDE, on the other hand, are functions of the statistics of the loading index $(L)$ 
and the rates of evolution of the internal variables:

$$
\begin{gathered}
N_{(1)_{i}}^{I V^{p l}}(\boldsymbol{\sigma}, \boldsymbol{q} ; t)=\left\langle L r_{i}(\boldsymbol{\sigma}, \boldsymbol{q} ; t)\right\rangle+ \\
\int_{0}^{t} d \tau \operatorname{Cov}_{0}\left[\frac{\partial}{\partial q_{j}}\left\{L r_{i}(\boldsymbol{\sigma}, \boldsymbol{q} ; t)\right\} ; L r_{j}(\boldsymbol{\sigma}, \boldsymbol{q} ; t-\tau)\right] \\
N_{(2)_{i j}}^{I V^{p l}}(\boldsymbol{\sigma}, \boldsymbol{q} ; t)=\int_{0}^{t} d \tau \operatorname{Cov}_{0}\left[\operatorname{Lr}_{i}(\boldsymbol{\sigma}, \boldsymbol{q} ; t) ; \operatorname{Lr}_{j}(\boldsymbol{\sigma}, \boldsymbol{q} ; t-\tau)\right]
\end{gathered}
$$

where $L$ may be expressed in terms of elastic modulus, yield function, plastic potential function, rate of strain, internal variables, and rates of evolution of internal variables as $[6]$ :

$$
L(\boldsymbol{\sigma}, \boldsymbol{q} ; t)=\frac{\frac{\partial F}{\partial \sigma_{o t}} D_{i j o t}^{e l} \dot{\epsilon}_{i j}}{\frac{\partial U}{\partial \sigma_{a b}} D_{a b c d}^{e l} \frac{\partial F}{\partial \sigma_{c d}}-\frac{\partial F}{\partial q_{n}} r_{n}}
$$

\section{Initial and Boundary Conditions}

Initial conditions for the governing FPK equations (Eqs. (2.1) and (2.2)) could be deterministic or probabilistic. A deterministic initial condition may be mathematically represented by a (multidimensional) Dirac delta function. It may be visualized as a (joint) probability density function with all the probabilities concentrated at a single point. Any (multivariate) probability density function may be used for a probabilistic initial condition.

Regarding the boundary conditions, a zero net flow of probability across the boundary is desirable. It can be achieved through reflecting barriers at the boundaries. Mathematically, a reflecting barrier at any point may be enforced by setting the probability current, $\zeta$, at that point to be equal to zero. Accordingly, the boundary 
conditions of the above Eqs. (2.1) and (2.2) can be written as follows:

$$
\begin{aligned}
\left.\zeta_{m n}(\boldsymbol{\sigma}, t)\right|_{\text {at boundaries }}= & \left\{N_{(1)_{m n}^{\sigma^{e q}}}(\boldsymbol{\sigma}, \boldsymbol{q} ; t) P(\boldsymbol{\sigma}, t)-\right. \\
& \left.\frac{\partial}{\partial \sigma_{a b}}\left[N_{(2)_{m n a b}^{\sigma^{e q}}}(\boldsymbol{\sigma}, \boldsymbol{q} ; t) P(\boldsymbol{\sigma}, t)\right]\right\}\left.\right|_{\text {at boundaries }}=0 \\
\left.\zeta_{i}(\boldsymbol{q}, t)\right|_{\text {at boundaries }}= & \left\{N_{(1)_{i}}^{I V^{e q}}(\boldsymbol{\sigma}, \boldsymbol{q} ; t) \widetilde{P}(\boldsymbol{q}, t)-\right. \\
& \left.\frac{\partial}{\partial q_{j}}\left[N_{(2)_{i j}}^{I V^{e q}}(\boldsymbol{\sigma}, \boldsymbol{q} ; t) \widetilde{P}(\boldsymbol{q}, t)\right]\right\}\left.\right|_{\text {at boundaries }}=0
\end{aligned}
$$

In the following the above general forms of the equations will be specialized for simulating two common laboratory constitutive experiments - unconfined compression test and unconsolidated undrained triaxial compression test - in geotechnical engineering.

\subsubsection{Unconfined Compression Test}

Unconfined compression test is a standard test in geotechnical engineering. It is a uniaxial test and, in a strain-controlled mode, is performed by applying axial strain to a cylindrical clayey soil specimen with no confining stress and observing the axial stresses corresponding to various strain levels [9].

\section{$\underline{\text { Linear Elastic Soil }}$}

If the soil is assumed to be linear elastic and to follow (rate form of) uniaxial Hooke's law, $\dot{\sigma}=E \dot{\epsilon}$, where $\dot{\epsilon}$ is the rate of axial strain, $\dot{\sigma}$ is the rate of axial stress, and $E$ 
is the (uncertain) Young's modulus, then the governing coupled, multivariate FPK equations (Eqs. (2.1) and (2.2)) will simplify to a single, univariate FPK equation (univariate form of Eq. (2.1)), as the internal variables do not exist for an uncertain elastic material:

$$
\begin{aligned}
\frac{\partial P(\sigma, t)}{\partial t} & =-\frac{\partial}{\partial \sigma}\left[N_{(1)}^{\sigma^{e l}} P(\sigma, t)\right]+\frac{\partial^{2}}{\partial \sigma^{2}}\left[N_{(2)}^{\sigma^{e l}}(t) P(\sigma, t)\right] \\
& =-N_{(1)}^{\sigma^{e l}} \frac{\partial P(\sigma, t)}{\partial \sigma}+N_{(2)}^{\sigma^{e l}}(t) \frac{\partial^{2} P(\sigma, t)}{\partial \sigma^{2}} \\
& =-\dot{\epsilon}\langle E\rangle \frac{\partial P(\sigma, t)}{\partial \sigma}+t \dot{\epsilon}^{2} \operatorname{Var}[E] \frac{\partial^{2} P(\sigma, t)}{\partial \sigma^{2}}
\end{aligned}
$$

Note that the coefficients, $N_{(1)}^{\sigma^{e l}}$ and $N_{(2)}^{\sigma^{e l}}$, of Eq. (2.18)) are constant with stress as specializing Eqs. (2.7) and (2.8) to uniaxial condition.

von Mises Elastic-Perfectly Plastic Soil

If the soil is assumed to follow a linear elastic-perfectly plastic constitutive law with a von Mises yield function, $F=\sigma-\sigma_{y}=0$, where $\sigma_{y}$ is the (uncertain) yield stress (an internal variable), then also the governing coupled, multivariate FPK equations (Eqs. (2.1) and (2.2)) will simplify to a single, univariate FPK equation (univariate form of Eq. (2.1)), as $\sigma_{y}$ does not evolve for an uncertain elastic-perfectly plastic material. However, unlike the linear elastic case, the coefficients of the governing univariate FPK equation will be functions of $\sigma$. Mathematically, the governing equation may be written as follows: 


$$
\begin{aligned}
\frac{\partial P(\sigma, t)}{\partial t}= & -\frac{\partial}{\partial \sigma}\left[N_{(1)}^{\sigma^{e q u}}(\sigma) P(\sigma, t)\right]+\frac{\partial^{2}}{\partial \sigma^{2}}\left[N_{(2)}^{\left.\sigma^{e q}\right)^{v M}}(\sigma, t) P(\sigma, t)\right] \\
= & -N_{(1)}^{\sigma^{e q u}}(\sigma) \frac{\partial P(\sigma, t)}{\partial \sigma}-P(\sigma, t) \frac{\partial N_{(1)}^{\sigma^{e q}}(\sigma)}{\partial \sigma}+ \\
& N_{(2)}^{\sigma^{e q^{v M}}}(\sigma, t) \frac{\partial^{2} P(\sigma, t)}{\partial \sigma^{2}}+P(\sigma, t) \frac{\partial^{2} N_{(2)}^{\sigma^{e q^{v M}}}(\sigma, t)}{\partial \sigma^{2}}+ \\
& 2 \frac{\partial P(\sigma, t)}{\partial \sigma} \frac{\partial N_{(2)}^{\sigma^{e q^{v M}}}(\sigma, t)}{\partial \sigma}
\end{aligned}
$$

The coefficients, $N_{(1)}^{\sigma^{e q^{v M}}}$ and $N_{(2)}^{\sigma^{e q u}}$, of Eq. (2.21) may be obtained by specializing Eqs. (2.3) and (2.4) to unaxial condition and to von Mises elastic-perfectly plastic constitutive equation as:

$$
\begin{aligned}
N_{(1)}^{\sigma^{e q u M}}(\sigma) & =\operatorname{Pr}[F<0](\sigma) N_{(1)}^{\sigma^{e l}}+\{1-\operatorname{Pr}[F<0]\}(\sigma) N_{(1)}^{\sigma^{p l^{v M}}} \\
& =\left\{1-\operatorname{Pr}\left[\sigma_{y} \leq \sigma\right]\right\}\langle E\rangle \dot{\epsilon} \\
N_{(2)}^{\sigma^{e q u}}(\sigma, t) & =\operatorname{Pr}[F<0](\sigma) N_{(2)}^{\sigma^{e l}}(t)+\{1-\operatorname{Pr}[F<0]\}(\sigma) N_{(2)}^{\sigma^{p l^{v M}}}(t) \\
& =t\left\{1-\operatorname{Pr}\left[\sigma_{y} \leq \sigma\right]\right\} \operatorname{Var}[E] \dot{\epsilon}^{2}
\end{aligned}
$$

Note that in the above Eqs. (2.23) and (2.25), the second additive terms on the r.h.s. are zero as the uniaxial plastic modulus for an elastic-perfectly plastic material is zero.

\subsubsection{Unconsolidated Undrained Triaxial Compression Test}

Unconsolidated undrained triaxial compression test is another standard test in geotechnical engineering. It is performed by first applying isotropic compression on a cylindrical saturated clayey soil specimen and then shearing the specimen by gradually 
increasing the axial strain, $\epsilon_{1}$, while keeping the radial confining pressure, $\sigma_{2}=\sigma_{3}$. During shearing, water is not allowed to escape the pores between the soil grains. As a result, the volumetric strain, $d \epsilon_{1}+d \epsilon_{2}+d \epsilon_{3}$, remains equal to zero during shearing; pore pressure and hence effective stress, however, vary.

Note that an unconsolidated undrained triaxial compression test may be analyzed in the principal stress-principal strain space if the soil is assumed to be of linear elastic or elastic-perfectly plastic type.

\section{$\underline{\text { Linear Elastic Soil }}$}

In the principal stress space, if the rate of principal stress, $\dot{\sigma}_{i}$ and the rate of principal strain, $\dot{\epsilon}_{j}$ are related through the (rate form of) generalized Hooke's law, $\dot{\sigma}_{i}=C_{i j} \dot{\epsilon}_{j}$, where $C_{i j}$ is the elastic moduli tensor, then the governing coupled FPK equations (Eqs. (2.1) and (2.2)) simplify to a single, but trivariate FPK equation:

$$
\frac{\partial P(\boldsymbol{\sigma}, t)}{\partial t}=-\frac{\partial}{\partial \sigma_{r}}\left[N_{(1)_{r}}^{\sigma^{e l}} P(\boldsymbol{\sigma}, t)\right]+\frac{\partial^{2}}{\partial \sigma_{r} \partial \sigma_{s}}\left[N_{(2)_{r s}}^{\sigma^{e l}}(t) P(\boldsymbol{\sigma}, t)\right]
$$

where $\boldsymbol{\sigma}=\left\{\sigma_{1}, \sigma_{2}, \sigma_{3}\right\}$ and the indices, $r$ and $s$, vary from 1 to 3. Note, however, that during the undrained triaxial shearing, $\sigma_{2}$ is always equal to $\sigma_{3}$. Hence the above trivariate FPK equation (Eq. (2.27)) may be simplified to a bivariate equation:

$$
\begin{aligned}
\frac{\partial P\left(\sigma_{1}, \sigma_{2}, t\right)}{\partial t}= & -N_{(1)_{1}}^{\sigma^{e l}} \frac{\partial P\left(\sigma_{1}, \sigma_{2}, t\right)}{\partial \sigma_{1}}-N_{(1)_{2}}^{\sigma^{e l}} \frac{\partial P\left(\sigma_{1}, \sigma_{2}, t\right)}{\partial \sigma_{2}}+ \\
& N_{(2)_{11}}^{\sigma^{e l}}(t) \frac{\partial^{2} P\left(\sigma_{1}, \sigma_{2}, t\right)}{\partial \sigma_{1}^{2}}+N_{(2)_{22}}^{\sigma^{e l}}(t) \frac{\partial^{2} P\left(\sigma_{1}, \sigma_{2}, t\right)}{\partial \sigma_{2}^{2}}+ \\
& 2 N_{(2)_{12}}^{\sigma^{e l}}(t) \frac{\partial^{2} P\left(\sigma_{1}, \sigma_{2}, t\right)}{\partial \sigma_{1} \partial \sigma_{2}}
\end{aligned}
$$


where the advection and diffusion coefficients may be obtained, by specializing Eqs. (2.7) and (2.8) to 2D principal space and by constraining them with no volume change condition, in the following general forms:

$$
\begin{aligned}
N_{(1)_{1}}^{\sigma^{e l}} & =\left\langle\frac{E}{1+\nu}\right\rangle \dot{\epsilon}_{1} \\
N_{(1)_{2}}^{\sigma^{e l}} & =-\frac{1}{2}\left\langle\frac{E}{1+\nu}\right\rangle \dot{\epsilon}_{1} \\
N_{(2)_{11}}^{\sigma^{e l}} & =t \operatorname{Var}\left[\frac{E}{1+\nu}\right] \dot{\epsilon}_{1}^{2} \\
N_{(2)_{22}}^{\sigma^{e l}} & =t \frac{1}{4} \operatorname{Var}\left[\frac{E}{1+\nu}\right] \dot{\epsilon}_{1}^{2} \\
N_{(2)_{12}}^{\sigma^{e l}} & =t \operatorname{Cov}\left[\frac{E}{1+\nu} ;-\frac{1}{2} \frac{E}{1+\nu}\right] \dot{\epsilon}_{1}^{2}
\end{aligned}
$$

where $\dot{\epsilon}_{1}$ is the rate of the first principal strain, which is also the axial strain.

von Mises Elastic-Perfectly Plastic Soil

In the case of a von Mises elastic-perfectly plastic soil with a yield criteria, $\mathrm{F}=\sqrt{3 J_{2}}-$ $\sigma_{y}=0$, where $J_{2}$ is the second invariant of the stress tensor, the form of governing FPK equation will be the same as that for the linear elastic soil (Eq. (2.28)), but both the advection and diffusion coefficients will be functions of stress:

$$
\frac{\partial P(\boldsymbol{\sigma}, t)}{\partial t}=-\frac{\partial}{\partial \sigma_{r}}\left[N_{(1)_{r}}^{\sigma^{e q^{v M}}}(\boldsymbol{\sigma}) P(\boldsymbol{\sigma}, t)\right]+\frac{\partial^{2}}{\partial \sigma_{r} \partial \sigma_{s}}\left[N_{(2)_{r s}}^{\sigma^{e q^{v M}}}(\boldsymbol{\sigma}, t) P(\boldsymbol{\sigma}, t)\right]
$$

where $\boldsymbol{\sigma}=\left\{\sigma_{1}, \sigma_{2}, \sigma_{3}\right\}$ and the indices, $r$ and $s$, vary from 1 to 3 . With $\sigma_{2}$ always equal to $\sigma_{3}$, Eq. (2.30) simplifies to a bivariate equation: 


$$
\begin{aligned}
& \frac{\partial P(\boldsymbol{\sigma}, t)}{\partial t}=-P(\boldsymbol{\sigma}, t) \frac{\partial N_{(1)_{1}}^{\sigma^{e q}}(\boldsymbol{\sigma})}{\partial \sigma_{1}}-N_{(1)_{1}}^{\sigma^{e q}}(\boldsymbol{\sigma}) \frac{\partial P(\boldsymbol{\sigma}, t)}{\partial \sigma_{1}}-P(\boldsymbol{\sigma}, t) \frac{\partial N_{(1)_{2}}^{\sigma^{e q M}}(\boldsymbol{\sigma})}{\partial \sigma_{2}}- \\
& N_{(1)_{2}}^{\sigma^{e q^{v M}}}(\boldsymbol{\sigma}) \frac{\partial P(\boldsymbol{\sigma}, t)}{\partial \sigma_{2}}+N_{(2)_{11}}^{\sigma^{e q^{v M}}}(\boldsymbol{\sigma}, t) \frac{\partial^{2} P(\boldsymbol{\sigma}, t)}{\partial \sigma_{1}^{2}}+P(\boldsymbol{\sigma}, t) \frac{\partial^{2} N_{(2)_{11}^{\sigma^{e q M}}}(\boldsymbol{\sigma}, t)}{\partial \sigma_{1}^{2}}+ \\
& 2 \frac{\partial N_{(2)_{11}^{\sigma_{11}}}^{\sigma^{v M}}(\boldsymbol{\sigma}, t)}{\partial \sigma_{1}} \frac{\partial P(\boldsymbol{\sigma}, t)}{\partial \sigma_{1}}+N_{(2)_{22}}^{\sigma^{e q} v M}(\boldsymbol{\sigma}, t) \frac{\partial^{2} P(\boldsymbol{\sigma}, t)}{\partial \sigma_{2}^{2}}+P(\boldsymbol{\sigma}, t) \frac{\partial^{2} N_{(2)_{22}}^{\sigma^{e q} v M}(\boldsymbol{\sigma}, t)}{\partial \sigma_{2}^{2}}+ \\
& 2 \frac{\partial N_{(2)_{22}}^{\sigma^{e q^{v M}}}(\boldsymbol{\sigma}, t)}{\partial \sigma_{2}} \frac{\partial P(\boldsymbol{\sigma}, t)}{\partial \sigma_{2}}+2 N_{(2)_{12}}^{\sigma^{e q}}(\boldsymbol{\sigma}, t) \frac{\partial^{2} P(\boldsymbol{\sigma}, t)}{\partial \sigma_{1} \partial \sigma_{2}}+2 \frac{\partial N_{(2)_{12} \sigma^{e q M}}^{v M}(\boldsymbol{\sigma}, t)}{\partial \sigma_{1}} \frac{\partial P(\boldsymbol{\sigma}, t)}{\partial \sigma_{2}}+ \\
& 2 \frac{\partial N_{(2)_{12}}^{\sigma^{e q}}(\boldsymbol{\sigma}, t)}{\partial \sigma_{2}} \frac{\partial P(\boldsymbol{\sigma}, t)}{\partial \sigma_{1}}+2 P(\boldsymbol{\sigma}, t) \frac{\partial^{2} N_{(2)_{12}}^{\sigma^{e v M}}(\boldsymbol{\sigma}, t)}{\partial \sigma_{1} \partial \sigma_{2}}
\end{aligned}
$$

where $\boldsymbol{\sigma}=\left\{\sigma_{1}, \sigma_{2}\right\}$ and the coefficients take the following forms:

$$
\begin{aligned}
& N_{(1)_{1}}^{\sigma^{e q M}}(\boldsymbol{\sigma})=\operatorname{Pr}[F<0](\boldsymbol{\sigma}) N_{(1)_{1}}^{\sigma^{e l}}+\{1-\operatorname{Pr}[F<0]\}(\boldsymbol{\sigma}) N_{(1)_{1}}^{\sigma^{p l^{v M}}}(\boldsymbol{\sigma}, t) \\
& =\left\{1-\operatorname{Pr}\left[\sigma_{y} \leq \sqrt{J_{2}}\right]\right\} N_{(1)_{1}}^{\sigma^{e l}} \\
& N_{(1)_{2}}^{\sigma^{e q M}}(\boldsymbol{\sigma})=\operatorname{Pr}[F<0](\boldsymbol{\sigma}) N_{(1)_{2}}^{\sigma^{e l}}+\{1-\operatorname{Pr}[F<0]\}(\boldsymbol{\sigma}) N_{(1)_{2}}^{\sigma^{p l^{v M}}}(\boldsymbol{\sigma}, t) \\
& =\left\{1-\operatorname{Pr}\left[\sigma_{y} \leq \sqrt{J_{2}}\right]\right\} N_{(1)_{2}}^{\sigma^{e l}} \\
& N_{(2)_{11}}^{\sigma^{e q M}}(\boldsymbol{\sigma}, t)=\operatorname{Pr}[F<0](\boldsymbol{\sigma}) N_{(2)_{11}}^{\sigma^{e l}}(t)+\{1-\operatorname{Pr}[F<0]\}(\boldsymbol{\sigma}) N_{(2)_{11}}^{\sigma^{p l}}(\boldsymbol{\sigma}, t) \\
& =\left\{1-\operatorname{Pr}\left[\sigma_{y} \leq \sqrt{J_{2}}\right]\right\} N_{(2)_{11}}^{\sigma^{e l}}(t) \\
& N_{(2)_{22}}^{\sigma^{e q} v}(\boldsymbol{\sigma}, t)=\operatorname{Pr}[F<0](\boldsymbol{\sigma}) N_{(2)_{22}}^{\sigma^{e l}}(t)+\{1-\operatorname{Pr}[F<0]\}(\boldsymbol{\sigma}) N_{(2)_{22}}^{\sigma^{p l}}(\boldsymbol{\sigma}, t) \\
& =\left\{1-\operatorname{Pr}\left[\sigma_{y} \leq \sqrt{J_{2}}\right]\right\} N_{(2)_{22}}^{\sigma^{e l}}(t) \\
& N_{(2)_{12}}^{\sigma^{e q}}(\boldsymbol{\sigma}, t)=\operatorname{Pr}[F<0](\boldsymbol{\sigma}) N_{(2)_{12}}^{\sigma^{e l}}(t)+\{1-\operatorname{Pr}[F<0]\}(\boldsymbol{\sigma}) N_{(2)_{12}}^{\sigma^{p l^{v M}}}(\boldsymbol{\sigma}, t) \\
& =\left\{1-\operatorname{Pr}\left[\sigma_{y} \leq \sqrt{J_{2}}\right]\right\} N_{(2)_{12}}^{\sigma^{e l}}(t)
\end{aligned}
$$


where $N_{(1)_{1}}^{\sigma^{e l}}, N_{(1)_{2}}^{\sigma^{e l}}, N_{(2)_{11}}^{\sigma^{e l}}, N_{(2)_{22}}^{\sigma^{e l}}$, and $N_{(2)_{12}}^{\sigma^{e l}}$ are given in Eq. (2.29). Note that the above coefficients are obtained by specializing Eqs. (2.3) and (2.4) to the 2D principal space, Eqs. (2.9) and (2.10) to the von Mises elastic-perfectly plastic yield condition and by applying the no volume change constraint. 


\section{CHAPTER III}

\section{SOLUTION ALGORITHMS FOR THE FOKKER-PLANCK-KOLMOGOROV EQUATIONS IN PROBABILISTIC ELASTO-PLASTICITY}

\subsection{Introduction}

The FPK equation is a linear deterministic partial differential equation (PDE). In general, there are several analytical and numerical techniques for solving PDEs. One of the simplest and oldest methods is the finite difference method (FDM). The advent of the finite difference technique in numerical applications began in the early 1950s and by the emergence of computers it became a convenient framework for solving the complex differential equations.

In these methods, derivatives in the PDE are approximated by linear combinations of function values (obtained from the Taylor series expansion) at the grid points. The domain is discretized in space and in time and the solution is approximated at the space or time points. Going from differential operator to a difference operator causes an error called the truncation error which can be reduced by using more terms of the Taylor series in approximation of the derivatives. There is another source of error in finite difference technique which is due to the discretization of the space and time domains. For an accurate solution the size of the subdomains should 
be small enough. Therefore, for large-scale computations, specially in several space dimensions, finite difference techniques are computationally very expensive.

For multi-dimensioal problems, spectral methods which are more accurate and computationally less expensive than finite difference techniques are increasingly being used for solving PDEs. Cornelius Lanczos [31] and C. W. Clenshaw [7] in the 1950s showed the power of Fourier series and Chebyshev polynomials in solving ordinary differential equations. In the 1970s, spectral methods were introduced again by Kreiss and Oliger [22] and Orszag and Israeli [41] for solving the PDEs in fluid mechanics. In general, spectral methods fall into three different categories:

1. Galerkin spectral method: In spectral methods, the numerical solution is expressed as a finite expansion of some set of basis functions. The spectral method is called Galerkin spectral method, if the PDE is written in terms of the coefficients of the global expansion. In this method, the basis functions need to satisfy some or all of the boundary conditions.

2. Spectral collocation method (also known as pseudospectral method): In this method, a set of gridpoints which are called collocation (or interpolation) points is used. This method demands that the differential equation be exactly satisfied at the collocation points.

3. Tau spectral method: This method is similar to the Galerkin method; however, the basis functions do not need to satisfy boundary conditions. 
Among these three methods, collocation methods are the most widely used spectral methods, mainly because they are able to treat nonlinear terms very easily. In this chapter, first the finite difference based numerical solution scheme for solving the FPK equation is discussed. Then a computationally efficient algorithms based on spectral collocation methods for solving a class of FPK PDEs that arises in probabilistic elasto-plasticity is presented. This class includes linear FPK PDEs in (stress) space and (pseudo) time - having time-dependent but space-independent, and both time- and space-dependent coefficients - with impulse initial conditions and reflecting boundary conditions. The versatility of the algorithms will be demonstrated in chapter 4 through solutions of both univariate and multivariate forms of the above types of PDEs.

\subsection{Finite Difference Techniques}

\subsubsection{Finite Difference Approximations}

Approximation of the First Derivatives

The Taylor series expansion of continuous function $u$ for any $\Delta x>0$ can be written as:

$$
u\left(x_{i}+\Delta x\right)=u\left(x_{i}\right)+\Delta x u^{\prime}\left(x_{i}\right)+\frac{\Delta x^{2}}{2 !} u^{\prime \prime}\left(x_{i}\right)+\frac{\Delta x^{3}}{3 !} u^{(3)}\left(x_{i}\right)+\cdots
$$

For the sake of simplicity, the first two terms of the Taylor expansion can be retained:

$$
u\left(x_{i}+\Delta x\right)=u\left(x_{i}\right)+\Delta x u^{\prime}\left(x_{i}\right)+O\left(\Delta x^{2}\right)
$$


In the above equation, the term $O\left(\Delta x^{2}\right)$ indicates that the error of the approximation is proportional to $\Delta x^{2}$.

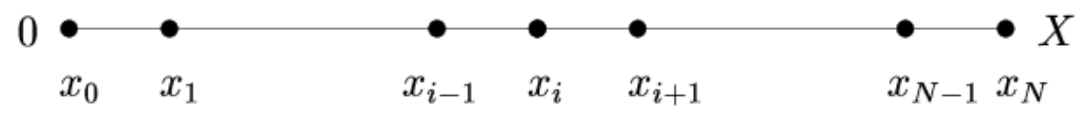

Figure 3.1: Finite difference grid used for discretizing univariate FPK equation

In the finite difference technique, the derivative of the function $u$ at the point $\mathrm{x}$ can be estimated as

$$
u^{\prime}\left(x_{i}\right)=\lim _{\Delta x \rightarrow 0} \frac{u\left(x_{i}+\Delta x\right)-u\left(x_{i}\right)}{\Delta x}
$$

where $\Delta x=x_{i+1}-x_{i}$, and it should be significantly small to get a good approximation of the derivative. This approximation of $u^{\prime}$ at point $x_{i}$ is known as the first order forward difference approximation of $u^{\prime}$. Likewise, the first order backward difference approximation of $u^{\prime}$ at point $x_{i}$ can be found.

$$
u\left(x_{i}-\Delta x\right)=u\left(x_{i}\right)-\Delta x u^{\prime}\left(x_{i}\right)+O\left(\Delta x^{2}\right)
$$

Therefore,

$$
u^{\prime}\left(x_{i}\right)=\lim _{\Delta x \rightarrow 0} \frac{u\left(x_{i}\right)-u\left(x_{i}-\Delta x\right)}{\Delta x}
$$

In order to improve the accuracy of the approximation, the central difference approximation can be defined using the points $x-\Delta x$ and $x+\Delta x$. 


$$
u^{\prime}\left(x_{i}\right)=\lim _{\Delta x \rightarrow 0} \frac{u\left(x_{i}+\Delta x\right)-u\left(x_{i}-\Delta x\right)}{2 \Delta x}
$$

Therefore, the first order derivatives can be approximated using forward difference, backward difference and central difference as follows:

Forward difference of order $O(\Delta x)$ :

$$
u^{\prime}\left(x_{i}\right) \approx \frac{u\left(x_{i}+\Delta x\right)-u\left(x_{i}\right)}{\Delta x}=\frac{u_{i+1}-u_{i}}{\Delta x}
$$

Backward difference of order $O(\Delta x)$ :

$$
u^{\prime}\left(x_{i}\right) \approx \frac{u\left(x_{i}\right)-u\left(x_{i}-\Delta x\right)}{\Delta x}=\frac{u_{i}-u_{i-1}}{\Delta x}
$$

Central difference of order $O(\Delta x)^{2}$ :

$$
u^{\prime}\left(x_{i}\right) \approx \frac{u\left(x_{i}+\Delta x\right)-u\left(x_{i}-\Delta x\right)}{2 \Delta x}=\frac{u_{i+1}-u_{i-1}}{2 \Delta x}
$$

Approximation of the Second Derivatives

As mentioned earlier, the Taylor expansions can be written as:

$$
\begin{aligned}
& u\left(x_{i}+\Delta x\right)=u\left(x_{i}\right)+\Delta x u^{\prime}\left(x_{i}\right)+\frac{\Delta x^{2}}{2 !} u^{\prime \prime}\left(x_{i}\right)+\frac{\Delta x^{3}}{3 !} u^{(3)}\left(x_{i}\right)+O\left(\Delta x^{4}\right) \\
& u\left(x_{i}-\Delta x\right)=u\left(x_{i}\right)-\Delta x u^{\prime}\left(x_{i}\right)+\frac{\Delta x^{2}}{2 !} u^{\prime \prime}\left(x_{i}\right)-\frac{\Delta x^{3}}{3 !} u^{(3)}\left(x_{i}\right)+O\left(\Delta x^{4}\right)
\end{aligned}
$$

Therefore, the second-order derivatives of order $O(\Delta x)^{2}$ can be approximated as:

$$
u^{\prime \prime}\left(x_{i}\right) \approx \frac{u\left(x_{i}+\Delta x\right)-2 u\left(x_{i}\right)+u\left(x_{i}-\Delta x\right)}{(\Delta x)^{2}}=\frac{u_{i+1}-2 u_{i}+u_{i-1}}{(\Delta x)^{2}}
$$


Approximation of the Mixed Derivatives

Approximation of the mixed derivatives can be found as follows:

$$
\frac{\partial^{2} u}{\partial x \partial y}=\frac{\partial}{\partial x}\left(\frac{\partial u}{\partial y}\right)=\frac{\partial}{\partial y}\left(\frac{\partial u}{\partial x}\right)
$$

Therefore,

$$
\frac{\partial^{2} u}{\partial x \partial y}=\frac{\left(\frac{\partial u}{\partial y}\right)_{i+1, j}-\left(\frac{\partial u}{\partial y}\right)_{i-1, j}}{2 \Delta x}+O(\Delta x)^{2}
$$

where,

$$
\left(\frac{\partial u}{\partial y}\right)_{i+1, j}=\frac{u_{i+1, j+1}-u_{i+1, j-1}}{2 \Delta y}
$$

and,

$$
\left(\frac{\partial u}{\partial y}\right)_{i-1, j}=\frac{u_{i-1, j+1}-u_{i-1, j-1}}{2 \Delta y}
$$

In the above equations, $\Delta x=x_{i+1}-x_{i}$, and $\Delta y=y_{i+1}-y_{i}$ (Figure 3.2). Therefore Eq. (3.13) can be rewritten as:

$$
\left(\frac{\partial^{2} u}{\partial x \partial y}\right)_{i, j}=\frac{u_{i+1, j+1}-u_{i+1, j-1}-u_{i-1, j+1}+u_{i-1, j-1}}{4 \Delta x \Delta y}+O\left[(\Delta x)^{2},(\Delta y)^{2}\right]
$$

In the following the finite difference technique is used to find the solution of the governing univariate and bivariate FPK equations.

\subsubsection{Univariate Fokker-Planck Kolmogorov Equation}

The univariate FPK equation which can be used for simulating the stress-strain behavior of clayey soils under the unconfined compression test (for both linear elastic 


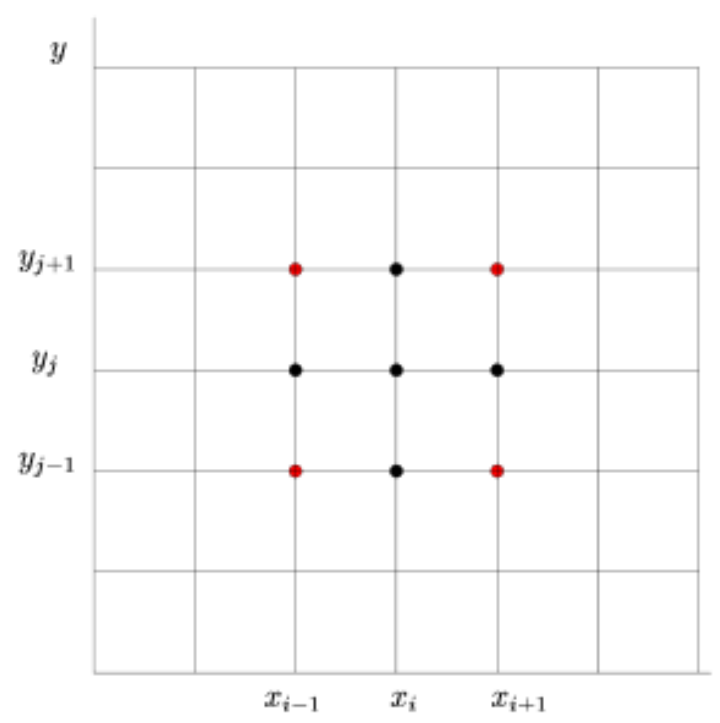

Figure 3.2: Finite difference grid used for discretizing bivariate FPK equation

and von Mises elastic-perfectly plastic soils) can be written as:

$$
\begin{aligned}
\frac{\partial P(\sigma, t)}{\partial t}= & -\frac{\partial}{\partial \sigma}\left[N_{(1)}^{\sigma^{e q}}(\sigma) P(\sigma, t)\right]+\frac{\partial^{2}}{\partial \sigma^{2}}\left[N_{(2)}^{\sigma^{e q}}(\sigma, t) P(\sigma, t)\right] \\
= & -N_{(1)}^{\sigma^{e q}}(\sigma) \frac{\partial P(\sigma, t)}{\partial \sigma}-P(\sigma, t) \frac{\partial N_{(1)}^{\sigma^{e q}}(\sigma)}{\partial \sigma}+ \\
& N_{(2)}^{\sigma^{e q}}(\sigma, t) \frac{\partial^{2} P(\sigma, t)}{\partial \sigma^{2}}+P(\sigma, t) \frac{\partial^{2} N_{(2)}^{\sigma^{e q}}(\sigma, t)}{\partial \sigma^{2}}+ \\
& 2 \frac{\partial P(\sigma, t)}{\partial \sigma} \frac{\partial N_{(2)}^{\sigma^{e q}}(\sigma, t)}{\partial \sigma}
\end{aligned}
$$

As discussed in chapter 2 (Eq. (2.16)), the reflective boundary condition can be expressed as follows:

$$
-N_{(1)}^{\sigma^{e q}}(\sigma) P(\sigma, t)+\frac{\partial}{\partial \sigma}\left[N_{(2)}^{\sigma^{e q}}(\sigma) P(\sigma, t)\right]=0
$$

or,

$$
-N_{(1)}^{\sigma^{e q}}(\sigma) P(\sigma, t)+N_{(2)}^{\sigma^{e q}}(\sigma) \frac{\partial P(\sigma, t)}{\partial \sigma}+P(\sigma, t) \frac{\partial N_{(2)}^{\sigma^{e q}}(\sigma)}{\partial \sigma}=0
$$


The finite difference method defines a mesh with spacing $d \sigma$ between grid points in the $\sigma$ direction. Therefore, by using the central difference formula, Eq. (3.19) can be written as:

$$
\begin{aligned}
& \frac{\partial P_{i}}{\partial t}=-N_{(1)_{i}}^{\sigma^{e q}}\left(\frac{P_{i+1}-P_{i-1}}{2 d \sigma}\right)-P_{i}\left(\frac{N_{(1)_{i+1}}^{\sigma^{e q}}-N_{(1)_{i-1}}^{\sigma^{e q}}}{2 d \sigma}\right)+ \\
& N_{(2)_{i}}^{\sigma^{e q}}\left(\frac{P_{i+1}-2 P_{i}+P_{i-1}}{\left(d \sigma^{2}\right)}\right)+2\left(\frac{P_{i+1}-P_{i-1}}{2 d \sigma}\right)\left(\frac{N_{(2)_{i+1}}^{\sigma^{e q}}-N_{(2)_{i-1}}^{\sigma^{e q}}}{2 d \sigma}\right)+ \\
& P_{i}\left(\frac{N_{(2)_{i+1}}^{\sigma^{e q}}-2 N_{(2)_{i}}^{\sigma^{e q}}+N_{(2)_{i-1}}^{\sigma^{e q}}}{\left(d \sigma^{2}\right)}\right)
\end{aligned}
$$

And the boundary condition would be as follows:

$$
-N_{(1)_{i}}^{\sigma^{e q}} P_{i}+N_{(2)_{i}}^{\sigma^{e q}}\left(\frac{P_{i+1}-P_{i-1}}{2 d \sigma}\right)+P_{i}\left(\frac{N_{(2)_{i+1}}^{\sigma^{e q}}-N_{(2)_{i-1}}^{\sigma^{e q}}}{2 d \sigma}\right)=0
$$

It should be noted that at the boundary at the left side Figure 3.3, forward difference and at the boundary at the right side, backward difference have been used.

$$
\begin{gathered}
u^{\prime}\left(x_{0}\right) \approx \frac{u\left(x_{1}\right)-u\left(x_{0}\right)}{\Delta x} \\
u^{\prime}\left(x_{n}\right) \approx \frac{u\left(x_{n}\right)-u\left(x_{n-1}\right)}{\Delta x}
\end{gathered}
$$

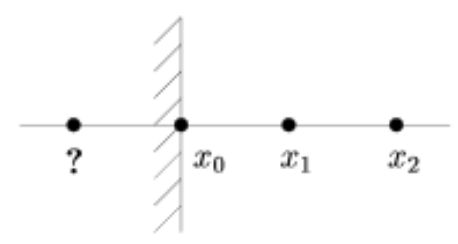

Figure 3.3: Forward difference is used for the boundary at the left side 
Therefore,

$$
\begin{array}{r}
-N_{(1)_{1}}^{\sigma^{e q}} P_{1}+N_{(2)_{1}}^{\sigma^{e q}}\left(\frac{P_{2}-P_{1}}{d \sigma}\right)+P_{1}\left(\frac{N_{(2)_{2}}^{\sigma^{e q}}-N_{(2)_{1}}^{\sigma^{e q}}}{d \sigma}\right)=0 \\
-N_{(1)_{N}}^{\sigma^{e q}} P_{N}+N_{(2)_{N}}^{\sigma^{e q}}\left(\frac{P_{N}-P_{N-1}}{d \sigma}\right)+P_{N}\left(\frac{N_{(2)_{N}}^{\sigma^{e q}}-N_{(2)_{N-1}}^{\sigma^{e q}}}{d \sigma}\right)=0
\end{array}
$$

The above algorithm is implemented in the $\mathrm{C}++$ programming language. By discretizing the stress domain and using the finite difference technique, a set of linear simultaneous ordinary differential equation (ODE) systems is obtained. Then, these linear simultaneous ODEs are solved using SUNDIALS library [21]. SUNDIALS library uses the CVODE solver for solving the ODE systems. The method used in CVODE is variable-order, variable-step multistep method, and it includes the Adams-Moulton formula, with order varying between 1 to 12 .

\subsubsection{Bivariate Fokker-Planck Kolmogorov Equation}

The finite difference technique discussed in the previous section, can be extended to the two-dimensional space for solving the bivariate FPK PDE. The bivariate FPK PDE which can be used for simulating the stress-strain behavior of clayey soils under the unconsolidated undrained triaxial compression test (for both linear elastic and von Mises elastic-perfectly plastic soils) can be written as:

$$
\begin{aligned}
& \frac{\partial P(\boldsymbol{\sigma}, t)}{\partial t}=-\frac{\partial}{\partial \sigma_{1}}\left[N_{(1)_{1}}^{\sigma^{e q}} P(\boldsymbol{\sigma}, t)\right]-\frac{\partial}{\partial \sigma_{2}}\left[N_{(1)_{2}}^{\sigma^{e q}} P(\boldsymbol{\sigma}, t)\right]+ \\
& \frac{\partial^{2}}{\partial \sigma_{1}^{2}}\left[N_{(2)_{11}}^{\sigma^{e q}} P(\boldsymbol{\sigma}, t)\right]+\frac{\partial^{2}}{\partial \sigma_{2}^{2}}\left[N_{(2)_{22}}^{\sigma^{e q}} P(\boldsymbol{\sigma}, t)\right]+2 \frac{\partial^{2}}{\partial \sigma_{1} \partial \sigma_{2}}\left[N_{(2)_{12}}^{\sigma^{e q}} P(\boldsymbol{\sigma}, t)\right]
\end{aligned}
$$


or in an extended form as:

$$
\begin{gathered}
\frac{\partial P(\boldsymbol{\sigma}, t)}{\partial t}=-P(\boldsymbol{\sigma}, t) \frac{\partial N_{(1)_{1}}^{\sigma^{e q}}(\boldsymbol{\sigma})}{\partial \sigma_{1}}-N_{(1)_{1}}^{\sigma^{e q}}(\boldsymbol{\sigma}) \frac{\partial P(\boldsymbol{\sigma}, t)}{\partial \sigma_{1}}-P(\boldsymbol{\sigma}, t) \frac{\partial N_{(1)_{2}}^{\sigma^{e q}}(\boldsymbol{\sigma})}{\partial \sigma_{2}}- \\
N_{(1)_{2}}^{\sigma^{e q}}(\boldsymbol{\sigma}) \frac{\partial P(\boldsymbol{\sigma}, t)}{\partial \sigma_{2}}+N_{(2)_{11}}^{\sigma^{e q}}(\boldsymbol{\sigma}, t) \frac{\partial^{2} P(\boldsymbol{\sigma}, t)}{\partial \sigma_{1}^{2}}+P(\boldsymbol{\sigma}, t) \frac{\partial^{2} N_{(2)_{11} \sigma^{e q}}(\boldsymbol{\sigma}, t)}{\partial \sigma_{1}^{2}}+ \\
2 \frac{\partial N_{(2)_{11}}^{\sigma^{e q}}(\boldsymbol{\sigma}, t)}{\partial \sigma_{1}} \frac{\partial P(\boldsymbol{\sigma}, t)}{\partial \sigma_{1}}+N_{(2)_{22}}^{\sigma^{e q}}(\boldsymbol{\sigma}, t) \frac{\partial^{2} P(\boldsymbol{\sigma}, t)}{\partial \sigma_{2}^{2}}+P(\boldsymbol{\sigma}, t) \frac{\partial^{2} N_{(2)_{22}}^{\sigma^{e q}}(\boldsymbol{\sigma}, t)}{\partial \sigma_{2}^{2}}+ \\
2 \frac{\partial N_{(2)_{22}}^{\sigma^{e q}}(\boldsymbol{\sigma}, t)}{\partial \sigma_{2}} \frac{\partial P(\boldsymbol{\sigma}, t)}{\partial \sigma_{2}}+2 N_{(2)_{12}}^{\sigma^{e q}}(\boldsymbol{\sigma}, t) \frac{\partial^{2} P(\boldsymbol{\sigma}, t)}{\partial \sigma_{1} \partial \sigma_{2}}+2 \frac{\partial N_{(2)_{12}}^{\sigma^{e q}}(\boldsymbol{\sigma}, t)}{\partial \sigma_{1}} \frac{\partial P(\boldsymbol{\sigma}, t)}{\partial \sigma_{2}}+ \\
2 \frac{\partial N_{(2)_{12}}^{\sigma^{e q}}(\boldsymbol{\sigma}, t)}{\partial \sigma_{2}} \frac{\partial P(\boldsymbol{\sigma}, t)}{\partial \sigma_{1}}+2 P(\boldsymbol{\sigma}, t) \frac{\partial^{2} N_{(2)_{12}}^{\sigma^{e q}}(\boldsymbol{\sigma}, t)}{\partial \sigma_{1} \partial \sigma_{2}}
\end{gathered}
$$

where $\boldsymbol{\sigma}=\left\{\sigma_{1}, \sigma_{2}\right\}$. As discussed in chapter 3, boundary conditions can be expressed as follows:

$$
\begin{aligned}
& -N_{(1)_{1}}^{\sigma^{e q}} P(\boldsymbol{\sigma}, t)+\frac{\partial}{\partial \sigma_{1}}\left\{N_{(2)_{11}}^{\sigma^{e q}} P(\boldsymbol{\sigma}, t)\right\}+\frac{\partial}{\partial \sigma_{2}}\left\{N_{(2)_{12}}^{\sigma^{e q}} P(\boldsymbol{\sigma}, t)\right\}=0 \\
& -N_{(1)_{2}}^{\sigma^{e q}} P(\boldsymbol{\sigma}, t)+\frac{\partial}{\partial \sigma_{1}}\left\{N_{(2)_{12}}^{\sigma^{e q}} P(\boldsymbol{\sigma}, t)\right\}+\frac{\partial}{\partial \sigma_{2}}\left\{N_{(2)_{22}}^{\sigma^{e q}} P(\boldsymbol{\sigma}, t)\right\}=0
\end{aligned}
$$

The above equations can be extended as:

$$
\begin{aligned}
& -N_{(1)_{1}}^{\sigma^{e q}} P(\boldsymbol{\sigma}, t)+\left\{N_{(2)_{11}}^{e q} \frac{\partial P(\boldsymbol{\sigma}, t)}{\partial \sigma_{1}}+P(\boldsymbol{\sigma}, t) \frac{\partial N_{(2)_{11}}^{e q}}{\partial \sigma_{1}}\right\}+ \\
& \left\{N_{(2)_{12}}^{e q} \frac{\partial P(\boldsymbol{\sigma}, t)}{\partial \sigma_{2}}+P(\boldsymbol{\sigma}, t) \frac{\partial N_{(2)_{12}}^{\sigma^{e q}}}{\partial \sigma_{2}}\right\}=0 \\
& -N_{(1)_{2}}^{\sigma^{e q}} P(\boldsymbol{\sigma}, t)+\left\{N_{(2)_{12}}^{\sigma^{e q}} \frac{\partial P(\boldsymbol{\sigma}, t)}{\partial \sigma_{1}}+P(\boldsymbol{\sigma}, t) \frac{\partial N_{(2)_{12}}^{\sigma^{e q}}}{\partial \sigma_{1}}\right\}+ \\
& \left\{N_{(2)_{22}}^{\sigma^{e q}} \frac{\partial P(\boldsymbol{\sigma}, t)}{\partial \sigma_{2}}+P(\boldsymbol{\sigma}, t) \frac{\partial N_{(2)_{22}}^{\sigma^{e q}}}{\partial \sigma_{2}}\right\}=0
\end{aligned}
$$

The finite difference method defines a mesh with spacing $d \sigma_{1}$ and $d \sigma_{2}$ between grid points in the $\sigma_{1}$ and $\sigma_{2}$ directions, respectively. Therefore, by using the central 
difference formula, Eq. (3.32) can be written as:

$$
\frac{\partial P_{(i, j)}}{\partial t}=-\left(A C_{1}+A C_{2}\right)+\left(D C_{11}+D C_{22}+2 D C_{12}\right)
$$

where, $A C_{r}$ are the advection coefficients and $D C_{r s}$ are the diffusion coefficients and can be written as:

$$
\begin{aligned}
& A C_{1}=P_{(i, j)}\left(\frac{\left.N_{(1)_{1(i+1, j)}^{\sigma^{e q}}}^{\sigma_{(1)_{1(i-1, j)}}}\right)-N_{(1)_{1(i, j)}}^{\sigma^{e q}}}{2 d \sigma_{1}}\left(\frac{P_{(i+1, j)}-P_{(i-1, j)}}{2 d \sigma_{1}}\right)\right. \\
& A C_{2}=P_{(i, j)}\left(\frac{N_{(1)_{1(i, j+1)}^{\sigma^{e q}}}^{\sigma_{1}}-N_{(1)_{1(i, j-1)}}^{\sigma^{e q}}}{2 d \sigma_{2}}\right)-N_{(1)_{2(i, j)}}^{\sigma^{e q}}\left(\frac{P_{(i, j+1)}-P_{(i, j-1)}}{2 d \sigma_{2}}\right) \\
& D C_{11}=N_{(2)_{11(i, j)}^{\sigma^{e q}}}\left(\frac{P_{(i+1, j)}-2 P_{(i, j)}+P_{(i-1, j)}}{\left(d \sigma_{1}\right)^{2}}\right)+ \\
& P_{(i, j)}\left(\frac{N_{(2)_{11(i+1, j)}^{\sigma^{e q}}}-2 N_{(2)_{11(i, j)}^{\sigma^{e q}}}+N_{(2)_{11(i-1, j)}^{\sigma^{e q}}}}{\left(d \sigma_{1}\right)^{2}}\right)+ \\
& 2\left(\frac{N_{(2)_{11(i+1, j)}^{\sigma^{e q}}}-N_{(2)_{11(i-1, j)}^{\sigma^{e q}}}}{2 d \sigma_{1}}\right)\left(\frac{P_{(i+1, j)}-P_{(i-1, j)}}{2 d \sigma_{1}}\right) \\
& D C_{22}=N_{(2)_{22(i, j)}}^{\sigma^{e q}}\left(\frac{P_{(i, j+1)}-2 P_{(i, j)}+P_{(i, j-1)}}{\left(d \sigma_{2}\right)^{2}}\right)+ \\
& P_{(i, j)}\left(\frac{N_{(2)_{22(i, j+1)}^{\sigma^{e q}}}-2 N_{(2)_{22(i, j)}^{\sigma^{e q}}}+N_{(2)_{22(i, j-1)}^{\sigma^{e q}}}}{\left(d \sigma_{2}\right)^{2}}\right)+ \\
& 2\left(\frac{\left.N_{(2)_{22(i, j+1)}^{\sigma^{e q}}}-N_{(2)_{22(i, j-1)}^{\sigma^{e q}}}\right)}{2 d \sigma_{2}}\right)\left(\frac{P_{(i, j+1)}-P_{(i, j-1)}}{2 d \sigma_{2}}\right) \\
& D C_{12}=N_{(2)_{12(i, j)}^{\sigma^{e q}}}\left(\frac{P_{(i+1, j+1)}-P_{(i+1, j-1)}-P_{(i-1, j+1)}+P_{(i-1, j-1)}}{4 d \sigma_{1} d \sigma_{2}}\right)+ \\
& P_{(i, j)}\left(\frac{N_{(2)_{12(i+1, j+1)}}^{\sigma^{e q}}-N_{(2)_{12(i+1, j-1)}^{\sigma^{e q}}}-N_{(2)_{12(i-1, j+1)}}^{\sigma^{e q}}+N_{(2)_{12(i-1, j-1)}}^{\sigma^{e q}}}{4 d \sigma_{1} d \sigma_{2}}\right)+ \\
& \left(\frac{N_{(2)_{12(i+1, j)}}^{\sigma^{e q}}-N_{(2)_{12(i-1, j)}}^{\sigma^{e q}}}{2 d \sigma_{1}}\right)\left(\frac{P_{(i, j+1)}-P_{(i, j-1)}}{2 d \sigma_{2}}\right)+ \\
& \left(\frac{N_{(2)_{12(i, j+1)}^{\sigma^{e q}}}^{\sigma^{2}}-N_{(2)_{12(i, j-1)}}^{\sigma^{e q}}}{2 d \sigma_{2}}\right)\left(\frac{P_{(i+1, j)}-P_{(i-1, j)}}{2 d \sigma_{1}}\right)
\end{aligned}
$$


And the boundary conditions can be expressed as follows:

$$
\begin{aligned}
& -N_{(1)_{1(i, j)}^{\sigma^{e q}}} P_{(i, j)}+\left\{N_{(2)_{11(i, j)}^{\sigma^{e q}}}\left(\frac{P_{(i+1, j)}-P_{(i-1, j)}}{2 d \sigma_{1}}\right)+\right.
\end{aligned}
$$

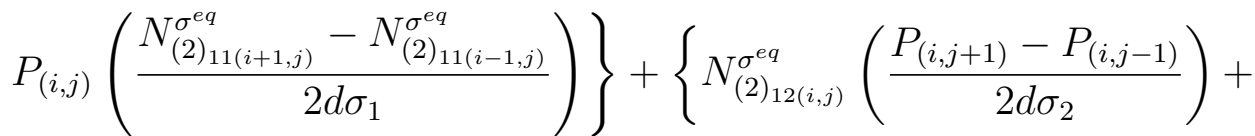

$$
\begin{aligned}
& \left.P_{(i, j)}\left(\frac{N_{(2)_{12(i, j+1)}}^{\sigma^{e q}}-N_{(2)_{11(i, j-1)}}^{\sigma^{e q}}}{2 d \sigma_{2}}\right)\right\}=0 \\
& -N_{(1)_{2(i, j)}}^{\sigma^{e q}} P_{(i, j)}+\left\{N_{(2)_{21(i, j)}^{\sigma^{e q}}}\left(\frac{P_{(i+1, j)}-P_{(i-1, j)}}{2 d \sigma_{1}}\right)+\right. \\
& \left.P_{(i, j)}\left(\frac{N_{(2)_{21(i+1, j)}^{\sigma^{e q}}}-N_{(2)_{21(i-1, j)}}^{e q}}{2 d \sigma_{1}}\right)\right\}+\left\{N_{(2)_{22(i, j)}}^{\sigma^{e q}}\left(\frac{P_{(i, j+1)}-P_{(i, j-1)}}{2 d \sigma_{2}}\right)+\right. \\
& \left.P_{(i, j)}\left(\frac{N_{(2)_{22(i, j+1)}}^{\sigma^{e q}}-N_{(2)_{22(i, j-1)}}^{\sigma^{e q}}}{2 d \sigma_{2}}\right)\right\}=0
\end{aligned}
$$

Similar to the 1-D case, forward difference formula is used at the boundaries at the left side, and backward difference formula is used at the boundaries at the right side. Therefore, the left boundaries can be written as:

$$
\begin{aligned}
& -N_{(1)_{1(1, j)}}^{\sigma^{e q}} P_{(1, j)}+\left\{N_{(2)_{11(1, j)}^{\sigma^{e q}}}\left(\frac{P_{(2, j)}-P_{(1, j)}}{d \sigma_{1}}\right)+\right. \\
& \left.P_{(1, j)}\left(\frac{N_{(2)_{11(2, j)}^{\sigma^{e q}}}-N_{(2)_{11(1, j)}^{\sigma^{e q}}}}{d \sigma_{1}}\right)\right\}+\left\{N_{(2)_{12(1, j)}^{\sigma^{e q}}}\left(\frac{P_{(1, j+1)}-P_{(1, j-1)}}{2 d \sigma_{2}}\right)+\right. \\
& \left.P_{(1, j)}\left(\frac{N_{(2)_{12(1, j+1)}}^{\sigma^{e q}}-N_{(2)_{12(1, j-1)}}^{\sigma^{e q}}}{2 d \sigma_{2}}\right)\right\}=0 \\
& -N_{(1)_{2(i, 1)}}^{\sigma^{e q}} P_{(i, 1)}+\left\{N_{(2)_{21(i, 1)}}^{\sigma^{e q}}\left(\frac{P_{(i+1,1)}-P_{(i-1,1)}}{2 d \sigma_{1}}\right)+\right.
\end{aligned}
$$

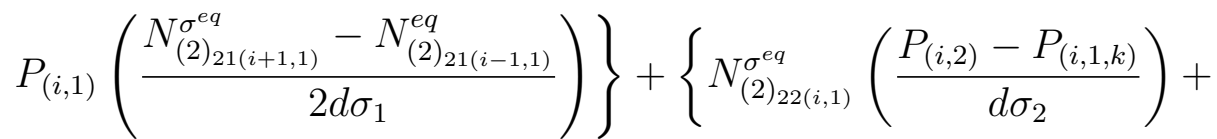

$$
\begin{aligned}
& \left.P_{(i, 1)}\left(\frac{N_{(2)_{22(i, 2)}}^{\sigma^{e q}}-N_{(2)_{22(i, 1)}}^{\sigma^{e q}}}{d \sigma_{2}}\right)\right\}=0
\end{aligned}
$$


Similarly, the right boundaries can be written as

$$
\begin{aligned}
& -N_{(1)_{1(N, j)}}^{\sigma^{e q}} P_{(N, j)}+\left\{N_{(2)_{11(N, j)}}^{\sigma^{e q}}\left(\frac{P_{(N, j)}-P_{(N-1, j)}}{d \sigma_{1}}\right)+\right. \\
& \left.P_{(N, j)}\left(\frac{N_{(2)_{11(N, j)}}^{\sigma^{e q}}-N_{(2)_{11(N-1, j)}}^{\sigma^{e q}}}{d \sigma_{1}}\right)\right\}+\left\{N_{(2)_{12(N, j)}^{\sigma^{e q}}}\left(\frac{P_{(N, j+1)}-P_{(N, j-1)}}{2 d \sigma_{2}}\right)+\right. \\
& \left.P_{(N, j)}\left(\frac{N_{(2)_{12(N, j+1)}}^{\sigma^{e q}}-N_{(2)_{12(N, j-1)}}^{\sigma^{e q}}}{2 d \sigma_{2}}\right)\right\}=0 \\
& -N_{(1)_{2(i, N)}}^{\sigma^{e q}} P_{(i, N)}+\left\{N_{(2)_{21(i, N)}}^{\sigma^{e q}}\left(\frac{P_{(i+1, N)}-P_{(i-1, N)}}{2 d \sigma_{1}}\right)+\right. \\
& \left.P_{(i, N)}\left(\frac{N_{(2)_{21(i+1, N)}}^{\sigma^{e q}}-N_{(2)_{21(i-1, N)}}^{\sigma^{e q}}}{2 d \sigma_{1}}\right)\right\}+\left\{N_{(2)_{22(i, N)}}^{\sigma^{e q}}\left(\frac{P_{(i, N)}-P_{(i, N-1)}}{d \sigma_{2}}\right)+\right. \\
& \left.P_{(i, N)}\left(\frac{N_{(2)_{22(i, N)}^{\sigma^{e q}}}-N_{(2)_{22(i, N-1)}}^{\sigma^{e q}}}{d \sigma_{2}}\right)\right\}=0
\end{aligned}
$$

The above algorithm is implemented in the $\mathrm{C}++$ programming language. By

discretizing the stress domain in each direction and using finite difference technique, a set of linear simultaneous ordinary differential equation (ODE) systems is obtained. Then, these linear simultaneous ODEs are solved using SUNDIALS library [21]. As mentioned earlier, SUNDIALS library uses the Adams-Moulton formula for solving the ODE systems.

\subsection{Fourier Spectral Method}

\subsubsection{Fourier Series}

The basic idea in using Fourier series for solving the differential equations is to assume that the unknown $u(x)$ can be approximated by a sum of $N+1$ basis functions like $\phi_{n}(x)([5]):$ 


$$
u(x) \approx u_{N}(x)=\sum_{0}^{N} a_{N} \phi_{n}(x)
$$

For solving a differential equation like $L u=f(x)$, we can substitute the series into the equation and find the residual function as follows:

$$
R\left(x: a_{0}, a_{1}, \cdots, a_{N}\right)=L u_{N}-f
$$

For the exact solution, the residual function should be equal to zero. Therefore, the series coefficients $a_{n}$ must be chosen in such a way that the residual function is minimized.

\subsubsection{Spectral Methods}

To solve a PDE with spectral methods, an approximate basis should be chosen. For instance, Fourier basis may be used for solving periodic problems, and polynomial basis for nonperiodic problems. It should be noted that function $f$ is a periodic function if there is an $a>0$ such that:

$$
f(x+a)=f(x)
$$

where $a$ is a period for $f$. The period is not unique. If there is more than one period, the smallest period is called the prime period of $f$. For example, $\sin x$ is a periodic function with prime period $2 \pi$.

Spectral methods are more accurate and memory-minimizing in compare to other methods, such as finite difference techniques. In the spectral methods, one high-order polynomial is used for whole domain. However, in the finite difference 
method, multiple overlapping low-order polynomials are used. Figure 3.4 shows the two types of numerical algorithms.

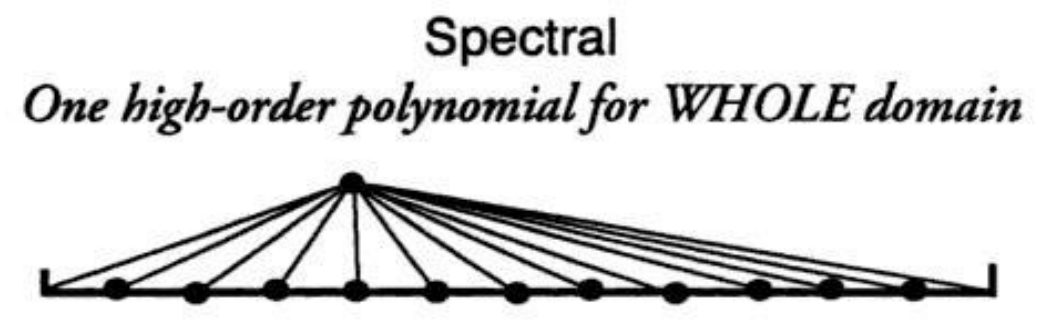

Finite Difference

Multiple Overlapping Low-Order Polynomials

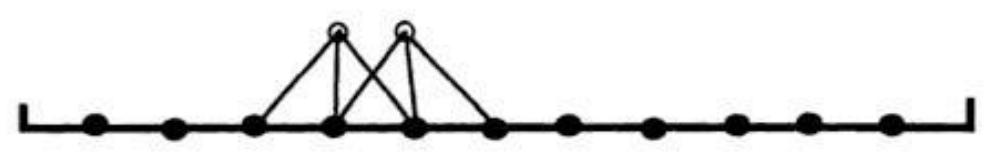

Figure 3.4: Spectral methods versus finite difference methods (modified from J. P. Boyd [5])

In spectral methods, the solution of a PDE may be approximated as:

$$
f(x)=\sum_{0}^{N} c_{n} \exp (i n x)
$$

where $i=\sqrt{-1}$. The first and second derivatives of $f(x)$ are approximated by $f^{\prime}(x)$ and $f^{\prime \prime}(x)$ which are given by:

$$
\begin{aligned}
& f^{\prime}(x)=\sum_{0}^{N} c_{n}(\text { in }) \exp (\text { inx }) \\
& f^{\prime \prime}(x)=\sum_{0}^{N} c_{n}\left(-n^{2}\right) \exp (\text { inx })
\end{aligned}
$$

The second step is to compute the spectral coefficients which can be obtained from the initial condition. In the following the spectral methods is used to develop 
computationally efficient algorithms for solving the governing univariate and bivariate FPK PDEs with space-independent but time-dependent coefficients, and both spaceand time-dependent coefficients.

\subsubsection{FPK PDE with Space-Independent but Time-Dependent Coefficients}

FPK PDEs with space-independent but time-dependent coefficients raise in probabilistic constitutive modeling of linear elastic materials. In this section, solution algorithm based on Fourier spectral approach for univariate and multivariate forms of the FPK PDEs with space-independent but time-dependent coefficients is presented.

The solution algorithm relies on first mapping the stress space of the governing PDE between 0 and $2 \pi$ using the change of coordinates rule, followed by approximating the solution of the PDE in the $2 \pi$-periodic domain by a finite Fourier series in the stress space and unknown time-dependent solution coefficients. Finally, the time-dependent solution coefficients are obtained from the initial condition through analytical integration.

$\underline{\text { Univariate Equation }}$

A univariate FPK PDE with space-independent but time-dependent coefficients was obtained in probabilistically modeling the stress-strain behavior of linear elastic soil under unconfined compression test as: 


$$
\begin{aligned}
\frac{\partial P(\sigma, t)}{\partial t} & =-\frac{\partial}{\partial \sigma}\left[N_{(1)}^{\sigma^{e l}} P(\sigma, t)\right]+\frac{\partial^{2}}{\partial \sigma^{2}}\left[N_{(2)}^{\sigma^{e l}}(t) P(\sigma, t)\right] \\
& =-N_{(1)}^{\sigma^{e l}} \frac{\partial P(\sigma, t)}{\partial \sigma}+N_{(2)}^{\sigma^{e l}}(t) \frac{\partial^{2} P(\sigma, t)}{\partial \sigma^{2}} \\
& =-\dot{\epsilon}\langle E\rangle \frac{\partial P(\sigma, t)}{\partial \sigma}+t \dot{\epsilon}^{2} \operatorname{Var}[E] \frac{\partial^{2} P(\sigma, t)}{\partial \sigma^{2}}
\end{aligned}
$$

Representation of the solution of Eq. (3.53) in terms of Fourier series necessitates mapping the domain of $\sigma$ between 0 and $2 \pi$. Hence, in the following, Eq. (3.53) will be re-written in terms of $\bar{\sigma}$, where $\bar{\sigma}$ varies from 0 to $2 \pi$. To that end, assuming that the original domain of $\sigma$ is $[a, b]$, the change of coordinates rule is utilized to first obtain the following relationship between $\sigma$ and $\bar{\sigma}$ (Figure 3.5):

$$
\bar{\sigma}=\frac{2 \pi}{b-a}(\sigma-a)
$$

Then, using the chain rule of differentiation, the derivatives appearing in Eq. (3.53) can be written in terms of $\bar{\sigma}$ as:

$$
\begin{gathered}
\frac{\partial P(\bar{\sigma}, t)}{\partial \sigma}=\frac{\partial P(\bar{\sigma}, t)}{\partial \bar{\sigma}} \frac{\partial \bar{\sigma}}{\partial \sigma}=\frac{2 \pi}{b-a} \frac{\partial P(\bar{\sigma}, t)}{\partial \bar{\sigma}} \\
\frac{\partial^{2} P(\bar{\sigma}, t)}{\partial \sigma^{2}}=\frac{\partial}{\partial \sigma}\left(\frac{2 \pi}{b-a} \frac{\partial P(\bar{\sigma}, t)}{\partial \bar{\sigma}}\right)=\left(\frac{2 \pi}{b-a}\right) \frac{\partial}{\partial \sigma}\left(\frac{\partial P(\bar{\sigma}, t)}{\partial \bar{\sigma}}\right) \\
=\left(\frac{2 \pi}{b-a}\right) \frac{\partial}{\partial \bar{\sigma}}\left(\frac{\partial P(\bar{\sigma}, t)}{\partial \bar{\sigma}}\right) \frac{\partial \bar{\sigma}}{\partial \sigma}=\left(\frac{2 \pi}{b-a}\right)^{2} \frac{\partial^{2} P(\bar{\sigma}, t)}{\partial \bar{\sigma}^{2}}
\end{gathered}
$$

Therefore, Eq. (3.53) can be re-written in terms of $\bar{\sigma}$ as:

$$
\frac{\partial P(\bar{\sigma}, t)}{\partial t}=-\dot{\epsilon}\langle E\rangle\left(\frac{2 \pi}{b-a}\right) \frac{\partial P(\bar{\sigma}, t)}{\partial \bar{\sigma}}+t \dot{\epsilon}^{2} \operatorname{Var}[E]\left(\frac{2 \pi}{b-a}\right)^{2} \frac{\partial^{2} P(\bar{\sigma}, t)}{\partial \bar{\sigma}^{2}}
$$




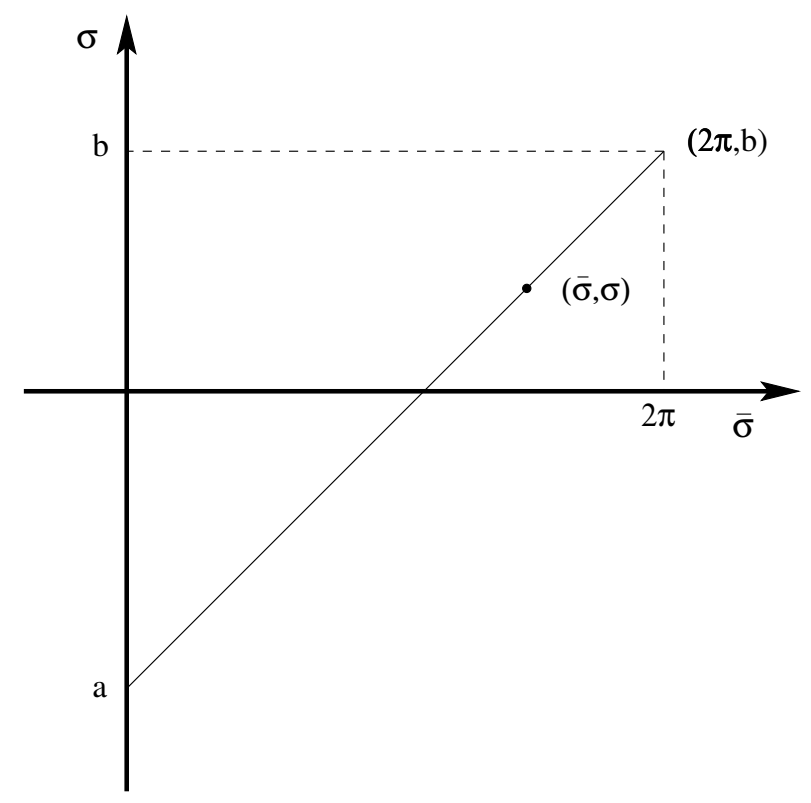

Figure 3.5: Mapping the stress space between 0 and $2 \pi$ using the change of coordinates rule

The solution of Eq. (3.57) may be written, in terms of a finite Fourier series in the $\bar{\sigma}$ space and unknown time-dependent coefficients, $\alpha_{n}$ as:

$$
P(\bar{\sigma}, t)=\sum_{n=-\frac{N}{2}}^{\frac{N}{2}} \alpha_{n}(t) e^{i n \bar{\sigma}}
$$

where $N$ is the number of Fourier terms. In obtaining the unknown coefficients, $\alpha_{n}$, substituting Eq. (3.58) in Eq. (3.57), it may be written that:

$$
\sum_{n=-\frac{N}{2}}^{\frac{N}{2}}\left[\alpha_{n}^{\prime}(t)+\dot{\epsilon}\langle E\rangle\left(\frac{2 \pi}{b-a}\right) i n \alpha_{n}+\dot{\epsilon}^{2} \operatorname{Var}[E]\left(\frac{2 \pi}{b-a}\right)^{2} t n^{2} \alpha_{n}\right] e^{i n \bar{\sigma}}=0
$$

Rearranging the terms, the above equation (Eq. (3.59)) may be re-written as:

$$
\frac{\alpha_{n}^{\prime}(t)}{\alpha_{n}(t)}=-\frac{2 \pi}{b-a}\left[\dot{\epsilon}^{2} \operatorname{Var}[E]\left(\frac{2 \pi}{b-a}\right) t n^{2}+\dot{\epsilon}\langle E\rangle \text { in }\right]
$$


Eq. (3.60) may be integrated to obtain the unknown coefficients, $\alpha_{n}$ :

$$
\alpha_{n}(t)=c_{n} e^{-\frac{2 \pi}{b-a}}\left[\frac{\dot{\epsilon}^{2} \operatorname{Var}[E]}{2}\left(\frac{2 \pi}{b-a}\right) t^{2} n^{2}+\dot{\epsilon}\langle E\rangle \text { int }\right]
$$

where $c_{n}$ are the constants of integration. Substituting Eq. (3.61) into Eq. (3.58), the general solution of Eq. (3.57) may be obtained as:

$$
P(\bar{\sigma}, t)=\sum_{n=-\frac{N}{2}}^{\frac{N}{2}} c_{n} e^{-\frac{2 \pi}{b-a}\left[\frac{\dot{\epsilon}^{2} \operatorname{Var}[E]}{2}\left(\frac{2 \pi}{b-a}\right) t^{2} n^{2}+\dot{\epsilon}\langle E\rangle i n t\right]+i n \bar{\sigma}}
$$

The solution of Eq. (3.57) may be obtained by evaluating $c_{n}$ from the initial condition.

\section{$\underline{\text { Initial Condition }}$}

Case 1: Deterministic initial condition

The deterministic initial condition for the unconfined compression test can be best represented by a Dirac delta function which mathematically can be written as:

$$
P(\sigma, 0)=\delta(\sigma-c)
$$

where $\sigma$ and $c$ are changing from $a$ to $b . \sigma$ and $c$ can be written in terms of $\bar{\sigma}$ and $\bar{c}$ as:

$$
\begin{aligned}
& \sigma=\frac{(b-a)}{2 \pi} \bar{\sigma}+a \\
& c=\frac{(b-a)}{2 \pi} \bar{c}+a
\end{aligned}
$$

Therefore,

$$
P(\bar{\sigma}, 0)=\delta(\bar{\sigma}-\bar{c})
$$

Substituting $t=0$ into Eq. (3.62) gives:

$$
\delta(\bar{\sigma}-\bar{c})=\sum_{n=-\frac{N}{2}}^{\frac{N}{2}} c_{n} e^{i \bar{\sigma} n}
$$


After multiplying both sides by $e^{-i m \bar{\sigma}}$ and then integrating both sides over $[0,2 \pi]$, Eq. (3.66) becomes:

$$
\int_{0}^{2 \pi} e^{-i \bar{\sigma} m} \delta(\bar{\sigma}-\bar{c}) d \bar{\sigma}=\left.\sum_{n=-\frac{N}{2}}^{\frac{N}{2}} c_{n} \frac{e^{i \bar{\sigma}(n-m)}}{n-m}\right|_{0} ^{2 \pi}
$$

Note that when $n \neq m$,

$$
\left.\frac{e^{i \bar{\sigma}(n-m)}}{n-m}\right|_{0} ^{2 \pi}=\frac{e^{i(n-m) \cdot 2 \pi}}{n-m}-\frac{1}{n-m}=\frac{1}{n-m}-\frac{1}{n-m}=0 \quad \text { when } n \neq m
$$

and, when $n=m$, Eq. (3.67) becomes:

$$
e^{-i \bar{\sigma} m} \delta(\bar{\sigma}-\bar{c})=c_{m} e^{i 0}=c_{m}
$$

Integrating over $[0,2 \pi]$ gives:

$$
2 \pi c_{m}=\int_{0}^{2 \pi} e^{-i \bar{\sigma} m} \delta(\bar{\sigma}-\bar{c}) d \bar{\sigma}=e^{-i \bar{c} m}
$$

where $d \bar{\sigma}=\frac{2 \pi}{b-a} d \sigma$. Therefore, the coefficients of the Fourier series are:

$$
c_{m}=\frac{1}{b-a} e^{-i \bar{c} m}
$$

or,

$$
c_{n}=\frac{1}{b-a} e^{-i \bar{c} n}
$$

Hence, the particular solution of Eq. (3.57) may be obtained as:

$$
P(\bar{\sigma}, t)=\sum_{n=-\frac{N}{2}}^{\frac{N}{2}} \frac{1}{b-a} \exp \left\{\left[-N_{1}^{e q}\left(\frac{2 \pi}{b-a}\right) i n t-\frac{t^{2}}{2}\left(\frac{2 \pi}{b-a}\right)^{2} N_{2}^{e q} n^{2}\right]-i \bar{c} n+i \bar{\sigma} n\right\}
$$

Note that, the initial condition for the unconfined compression test is centered at $\sigma=0$ as at the start of the test, the stress is zero (deterministic). Therefore for 
the unconfined compression test, Eq. (3.57) can be rewritten as:

$$
P(\bar{\sigma}, t)=\sum_{n=-\frac{N}{2}}^{\frac{N}{2}} \frac{1}{b-a} \exp \left\{\left[-N_{1}^{e q}\left(\frac{2 \pi}{b-a}\right) i n t-\frac{t^{2}}{2}\left(\frac{2 \pi}{b-a}\right)^{2} N_{2}^{e q} n^{2}\right]+i \bar{\sigma} n\right\}
$$

Case 2: Probabilistic initial condition

In a real problem, for solving a stochastic boundary value problem, a stochastic finite element framework is needed. In a finite element setting, the constitutive equation should be solved at each Gauss point. However, due to the uncertainty in the specific gravity of soils in computing the confining pressure, there are some initial uncertainties. Therefore, the initial condition would be probabilistic. The probabilistic initial condition should be replaced by a Gaussian function. Therefore, $P(\sigma, 0)$ can be written as:

$$
P(\sigma, 0)=\frac{1}{\sqrt{2 \pi} d} e^{-\frac{(\sigma-c)^{2}}{2 d^{2}}}
$$

where $\sigma, c$ and $d$ vary from $a$ to $b . \sigma$ and $c$ can be written in terms of $\bar{\sigma}$ and $\bar{c}$ using Eq. (3.64), and $d$ can be expressed int erms of $\bar{d}$ as:

$$
d=\frac{b-a}{2 \pi} \bar{d}
$$

Therefore, by substituting $\sigma, c$ and $d$ in terms of $\bar{\sigma}, \bar{c}$ and $\bar{d}$ into Eq. (3.75):

$$
P(\bar{\sigma}, 0)=\frac{1}{\sqrt{2 \pi}\left(\frac{b-a}{2 \pi} \bar{d}\right)} e^{\frac{-\left(\frac{b-a}{2 \pi} \bar{\sigma}+a-\frac{b-a}{2 \pi} \bar{c}+a\right)^{2}}{2\left(\frac{b-a}{2 \pi} \bar{d}\right)^{2}}}
$$


Simplifying Eq. (3.77) gives:

$$
P(\bar{\sigma}, 0)=\frac{1}{\sqrt{2 \pi}\left(\frac{b-a}{2 \pi} \bar{d}\right)} e^{\frac{-(\bar{\sigma}-\bar{c})^{2}}{2 \bar{d}^{2}}}
$$

or,

$$
P(\bar{\sigma}, 0)=\left(\frac{2 \pi}{b-a}\right) \frac{1}{\sqrt{2 \pi} \bar{d}} e^{\frac{-(\bar{\sigma}-\bar{c})^{2}}{2 \bar{d}^{2}}}
$$

Substituting $t=0$ into Eq. (3.62) gives:

$$
\left(\frac{2 \pi}{b-a}\right) \frac{1}{\sqrt{2 \pi} \bar{d}} e^{-\frac{(\bar{\sigma}-\bar{c})^{2}}{2 \bar{d}^{2}}}=\sum_{n=-\frac{N}{2}}^{\frac{N}{2}} c_{n} e^{i \bar{\sigma} n}
$$

Multiplying both side by $e^{-i \bar{\sigma} m}$,

$$
e^{-i \bar{\sigma} m}\left(\frac{2 \pi}{b-a}\right) \frac{1}{\sqrt{2 \pi} \bar{d}} e^{-\frac{(\bar{\sigma}-\bar{c})^{2}}{2 \bar{d}^{2}}}=\sum_{n=-\frac{N}{2}}^{\frac{N}{2}} c_{n} e^{i \bar{\sigma}(n-m)}
$$

Integrating both sides over $[0,2 \pi]$ gives:

$$
\int_{0}^{2 \pi} e^{-i \bar{\sigma} m}\left(\frac{2 \pi}{b-a}\right) \frac{1}{\sqrt{2 \pi} \bar{d}} e^{-\frac{(\bar{\sigma}-\bar{c})^{2}}{2 \bar{d}^{2}}} d \bar{\sigma}=\left.\sum_{n=-\frac{N}{2}}^{\frac{N}{2}} c_{n} \frac{e^{i \bar{\sigma}(n-m)}}{n-m}\right|_{0} ^{2 \pi}
$$

But

$$
\left.\frac{e^{i \bar{\sigma}(n-m)}}{n-m}\right|_{0} ^{2 \pi}=\frac{e^{i(n-m) \cdot 2 \pi}}{n-m}-\frac{1}{n-m}=\frac{1}{n-m}-\frac{1}{n-m}=0 \quad \text { when } n \neq m
$$

But when $n=m$, Eq. (3.81) becomes:

$$
e^{-i \bar{\sigma} m}\left(\frac{2 \pi}{b-a}\right) \frac{1}{\sqrt{2 \pi} \bar{d}} e^{-\frac{(\bar{\sigma}-\bar{c})^{2}}{2 \bar{d}^{2}}}=c_{m} e^{i 0}=c_{m}
$$

Integrating over $[0,2 \pi]$,

$$
2 \pi c_{m}=\int_{0}^{2 \pi}\left[e^{-i \bar{\sigma} m}\left(\frac{2 \pi}{b-a}\right) \frac{1}{\sqrt{2 \pi} \bar{d}} e^{-\frac{(\bar{\sigma}-\bar{c})^{2}}{2 \bar{d}^{2}}}\right] d \bar{\sigma}
$$


Therefore, $c_{m}$ can be computed as:

$$
c_{m}=\frac{1}{2 \pi} \int_{0}^{2 \pi}\left[e^{-i \bar{\sigma} m}\left(\frac{2 \pi}{b-a}\right) \frac{1}{\sqrt{2 \pi} \bar{d}} e^{-\frac{(\bar{\sigma}-\bar{c})^{2}}{2 \bar{d}^{2}}}\right] d \bar{\sigma}
$$

or,

$$
c_{n}=\frac{1}{2 \pi} \int_{0}^{2 \pi}\left[\left(\frac{2 \pi}{b-a}\right) \frac{1}{\sqrt{2 \pi} \bar{d}} e^{-\frac{(\bar{\sigma}-\bar{c})^{2}}{2 \bar{d}^{2}}} e^{-i \bar{\sigma} n}\right] d \bar{\sigma}
$$

Therefore, solution of Eq. (3.57) may be obtained as:

$$
\begin{array}{r}
P(\sigma, t)=\sum_{n=-\frac{N}{2}}^{\frac{N}{2}} \frac{1}{2 \pi}\left(\int_{0}^{2 \pi}\left[\left(\frac{2 \pi}{b-a}\right) \frac{1}{\sqrt{2 \pi} \bar{d}} \exp \left\{-\frac{(\bar{\sigma}-\bar{c})^{2}}{2 \bar{d}^{2}}-i \bar{\sigma} n\right\}\right] d \bar{\sigma}\right) \\
\exp \left\{-N_{1}^{e q}\left(\frac{2 \pi}{b-a}\right) i n t-\frac{t^{2}}{2}\left(\frac{2 \pi}{b-a}\right)^{2} N_{2}^{e q} n^{2}+i \bar{\sigma} n\right\}
\end{array}
$$

where $\bar{\sigma}, \bar{c}$ and $\bar{d}$ vary from 0 to $2 \pi$.

$c_{n}$ can be computed using any mathematical program (e.g., Mathematica, Matlab, etc.) as:

$$
\begin{aligned}
& c_{n}=\frac{1}{2 \pi} \int_{0}^{2 \pi}\left[\left(\frac{2 \pi}{b-a}\right) \frac{1}{\sqrt{2 \pi} \bar{d}} \exp \left\{-\frac{(\bar{\sigma}-\bar{c})^{2}}{2 \bar{d}^{2}}-i \bar{\sigma} n\right\}\right] d \bar{\sigma}= \\
& \frac{1}{2 \pi}\left(\frac{2 \pi}{b-a}\right) 0.5 e^{-\frac{1}{2} n\left(n \bar{d}^{2}+2 i \bar{c}\right)}\left[\operatorname{Erf}\left(\frac{\bar{c}-i \bar{d}^{2} n}{\sqrt{2} \bar{d}}\right)-\operatorname{Erf}\left(\frac{\bar{c}-2 \pi-i \bar{d}^{2} n}{\sqrt{2} \bar{d}}\right)\right]
\end{aligned}
$$

A note on the boundary conditions:

The general form of the reflecting boundary conditions presented in Eq. (2.16) simplifies to the following form for Eq. (3.57):

$$
\left.\left\{\dot{\epsilon}\langle E\rangle\left(\frac{2 \pi}{b-a}\right) P(\bar{\sigma}, t)-t \dot{\epsilon}^{2} \operatorname{Var}[E]\left(\frac{2 \pi}{b-a}\right)^{2} \frac{\partial P(\bar{\sigma}, t)}{\partial \bar{\sigma}}\right\}\right|_{\text {at boundaries }}=0
$$


Note, however, that:

$$
\begin{aligned}
\int_{0}^{2 \pi} \frac{\partial P(\bar{\sigma}, t)}{\partial t} d \bar{\sigma}= & \int_{0}^{2 \pi} \frac{\partial}{\partial t}\left[\frac { 1 } { b - a } \sum _ { n = - \frac { N } { 2 } } ^ { \frac { N } { 2 } } \operatorname { e x p } \left\{-\frac{2 \pi}{b-a}\left[\frac{\dot{\epsilon}^{2} \operatorname{Var}[E]}{2}\right.\right.\right. \\
& \left.\left.\left.\left(\frac{2 \pi}{b-a}\right) t^{2} n^{2}+\dot{\epsilon}\langle E\rangle i n t\right]+i n \bar{\sigma}\right\}\right] d \bar{\sigma} \\
= & 0
\end{aligned}
$$

Hence, the reflecting boundary conditions presented in Eq. (3.90) become the natural boundary conditions.

$\underline{\text { Multivariate Equation }}$

The solution scheme presented above for a univariate FPK PDE can be easily extended for a bivariate FPK PDE (Eq. (3.92)).

$$
\begin{aligned}
\frac{\partial P\left(\sigma_{1}, \sigma_{2}, t\right)}{\partial t}= & -N_{(1)_{1}}^{\sigma^{e l}} \frac{\partial P\left(\sigma_{1}, \sigma_{2}, t\right)}{\partial \sigma_{1}}-N_{(1)_{2}}^{\sigma^{e l}} \frac{\partial P\left(\sigma_{1}, \sigma_{2}, t\right)}{\partial \sigma_{2}}+ \\
& N_{(2)_{11}}^{\sigma^{e l}}(t) \frac{\partial^{2} P\left(\sigma_{1}, \sigma_{2}, t\right)}{\partial \sigma_{1}^{2}}+N_{(2)_{22}}^{\sigma^{e l}}(t) \frac{\partial^{2} P\left(\sigma_{1}, \sigma_{2}, t\right)}{\partial \sigma_{2}^{2}}+ \\
& 2 N_{(2)_{12}}^{\sigma^{e l}}(t) \frac{\partial^{2} P\left(\sigma_{1}, \sigma_{2}, t\right)}{\partial \sigma_{1} \partial \sigma_{2}}
\end{aligned}
$$

We will demonstrate this extension on Eq. (3.92), a bivariate FPK PDE with time-dependent but stress-independent coefficients, obtained in probabilistically modeling the stress-strain behavior of linear elastic soil under unconsolidated undrained triaxial compression test. Similar to the univariate case, mapping the domains of $\sigma_{1}$ and $\sigma_{2}$ from $\left[a_{1}, b_{1}\right]$ and $\left[a_{2}, b_{2}\right]$, respectively, to $[0,2 \pi]$, Eq. (3.92) may be written in terms of $\bar{\sigma}_{1}$ and $\bar{\sigma}_{2}$ as: 


$$
\begin{aligned}
\frac{\partial P\left(\bar{\sigma}_{1}, \bar{\sigma}_{2}, t\right)}{\partial t}= & -\dot{\epsilon}_{1}\langle\lambda\rangle\left(\frac{2 \pi}{b_{1}-a_{1}}\right) \frac{\partial P}{\partial \bar{\sigma}_{1}}-\dot{\epsilon}_{1}\left\langle-\frac{1}{2} \lambda\right\rangle\left(\frac{2 \pi}{b_{2}-a_{2}}\right) \frac{\partial P}{\partial \bar{\sigma}_{2}}+ \\
& t \dot{\epsilon}_{1}^{2} \operatorname{Var}[\lambda]\left(\frac{2 \pi}{b_{1}-a_{1}}\right)^{2} \frac{\partial^{2} P}{\partial \bar{\sigma}_{1}^{2}}+t \dot{\epsilon}_{1}^{2} \operatorname{Var}\left[-\frac{1}{2} \lambda\right]\left(\frac{2 \pi}{b_{2}-a_{2}}\right)^{2} \frac{\partial^{2} P}{\partial \bar{\sigma}_{2}^{2}}+ \\
& 2 t \dot{\epsilon}_{1}^{2} \operatorname{Cov}\left[\lambda ;-\frac{1}{2} \lambda\right]\left(\frac{2 \pi}{b_{1}-a_{1}}\right)\left(\frac{2 \pi}{b_{2}-a_{2}}\right) \frac{\partial^{2} P}{\partial \bar{\sigma}_{1} \partial \bar{\sigma}_{2}}
\end{aligned}
$$

where $\lambda=E /(1+\nu)$. The solution of Eq. (3.93) may be written in terms of a multidimensional finite Fourier series in $\left\{\overline{\sigma_{1}}, \overline{\sigma_{2}}\right\}$ space and unknown time-dependent coefficients, $\alpha_{m n}$ as:

$$
P\left(\bar{\sigma}_{1}, \bar{\sigma}_{2}, t\right)=\sum_{n=-\frac{N}{2}}^{\frac{N}{2}} \sum_{m=-\frac{M}{2}}^{\frac{M}{2}} \alpha_{m n}(t) e^{i n \bar{\sigma}_{1}} e^{i m \bar{\sigma}_{2}}
$$

where $n$ and $m$ are the numbers of Fourier terms in $\bar{\sigma}_{1}$ and $\bar{\sigma}_{2}$ spaces, respectively. Substituting Eq. (3.94) in Eq. (3.93), it may be written that:

$$
\begin{aligned}
& \sum_{n=-\frac{N}{2}}^{\frac{N}{2}} \sum_{m=-\frac{M}{2}}^{\frac{M}{2}}\left[\alpha_{m n}^{\prime}(t)+\right. \\
& \quad \dot{\epsilon}_{1}\langle\lambda\rangle\left(\frac{2 \pi}{b_{1}-a_{1}}\right) i n \alpha_{m n}(t)+\dot{\epsilon}_{1}\left\langle-\frac{1}{2} \lambda\right\rangle\left(\frac{2 \pi}{b_{2}-a_{2}}\right) i m \alpha_{m n}(t)+ \\
& \quad t \dot{\epsilon}_{1}^{2} \operatorname{Var}[\lambda]\left(\frac{2 \pi}{b_{1}-a_{1}}\right)^{2} n^{2} \alpha_{m n}(t)+t \dot{\epsilon}_{1}^{2} \operatorname{Var}\left[-\frac{1}{2} \lambda\right]\left(\frac{2 \pi}{b_{2}-a_{2}}\right)^{2} m^{2} \alpha_{m n}(t)+ \\
& \left.2 t \dot{\epsilon}_{1}^{2} \operatorname{Cov}\left[\lambda ;-\frac{1}{2} \lambda\right]\left(\frac{2 \pi}{b_{1}-a_{1}}\right)\left(\frac{2 \pi}{b_{2}-a_{2}}\right) n m \alpha_{m n}(t)\right] e^{i\left(n \bar{\sigma}_{1}+m \bar{\sigma}_{2}\right)}=0
\end{aligned}
$$


Rearranging the terms, Eq. (3.95) may be rewritten as:

$$
\begin{gathered}
\frac{\alpha_{m n}^{\prime}(t)}{\alpha_{m n}(t)}=-\left[\dot{\epsilon}_{1}\langle\lambda\rangle\left(\frac{2 \pi}{b_{1}-a_{1}}\right) i n+\dot{\epsilon}_{1}\left\langle-\frac{1}{2} \lambda\right\rangle\left(\frac{2 \pi}{b_{2}-a_{2}}\right) i m+\right. \\
t \dot{\epsilon}_{1}^{2} \operatorname{Var}[\lambda]\left(\frac{2 \pi}{b_{1}-a_{1}}\right)^{2} n^{2}+t \dot{\epsilon}_{1}^{2} \operatorname{Var}\left[-\frac{1}{2} \lambda\right]\left(\frac{2 \pi}{b_{2}-a_{2}}\right)^{2} m^{2}+ \\
\left.2 t \dot{\epsilon}_{1}^{2} \operatorname{Cov}\left[\lambda ;-\frac{1}{2} \lambda\right]\left(\frac{2 \pi}{b_{1}-a_{1}}\right)\left(\frac{2 \pi}{b_{2}-a_{2}}\right) n m\right]
\end{gathered}
$$

Eq. (3.96) may be integrated to obtain the unknown coefficients, $\alpha_{m n}$ :

$$
\begin{gathered}
\alpha_{m n}(t)=c_{m n} \exp \left[-\left\{\dot{\epsilon}_{1}\langle\lambda\rangle\left(\frac{2 \pi}{b_{1}-a_{1}}\right) i n t+\dot{\epsilon}_{1}\left\langle-\frac{1}{2} \lambda\right\rangle\left(\frac{2 \pi}{b_{2}-a_{2}}\right) i m t+\right.\right. \\
\dot{\epsilon}_{1}^{2} \operatorname{Var}[\lambda] \frac{t^{2}}{2}\left(\frac{2 \pi}{b_{1}-a_{1}}\right)^{2} n^{2}+\dot{\epsilon}_{1}^{2} \operatorname{Var}\left[-\frac{1}{2} \lambda\right] \frac{t^{2}}{2}\left(\frac{2 \pi}{b_{2}-a_{2}}\right)^{2} m^{2}+ \\
\left.\left.2 \dot{\epsilon}_{1}^{2} \operatorname{Cov}\left[\lambda ;-\frac{1}{2} \lambda\right] \frac{t^{2}}{2}\left(\frac{2 \pi}{b_{1}-a_{1}}\right)\left(\frac{2 \pi}{b_{2}-a_{2}}\right) n m\right\}\right]
\end{gathered}
$$

where $c_{m n}$ are the constants of integration. Substituting Eq. (3.97) into Eq. (3.94), the general solution of Eq. (3.93) may be obtained as:

$$
\begin{aligned}
& P\left(\bar{\sigma}_{1}, \bar{\sigma}_{2}, t\right)=\sum_{n=-\frac{N}{2}}^{\frac{N}{2}} \sum_{m=-\frac{M}{2}}^{\frac{M}{2}} c_{m n} . \\
& \quad \exp \left[-\left\{\dot{\epsilon}_{1}\langle\lambda\rangle\left(\frac{2 \pi}{b_{1}-a_{1}}\right) i n t+\dot{\epsilon}_{1}\left\langle-\frac{1}{2} \lambda\right\rangle\left(\frac{2 \pi}{b_{2}-a_{2}}\right) i m t+\right.\right. \\
& \dot{\epsilon}_{1}^{2} \operatorname{Var}[\lambda] \frac{t^{2}}{2}\left(\frac{2 \pi}{b_{1}-a_{1}}\right)^{2} n^{2}+\dot{\epsilon}_{1}^{2} \operatorname{Var}\left[-\frac{1}{2} \lambda\right] \frac{t^{2}}{2}\left(\frac{2 \pi}{b_{2}-a_{2}}\right)^{2} m^{2}+ \\
& \left.\left.2 \dot{\epsilon}_{1}^{2} \operatorname{Cov}\left[\lambda ;-\frac{1}{2} \lambda\right] \frac{t^{2}}{2}\left(\frac{2 \pi}{b_{1}-a_{1}}\right)\left(\frac{2 \pi}{b_{2}-a_{2}}\right) n m\right\}+i\left(n \bar{\sigma}_{1}+m \bar{\sigma}_{2}\right)\right]
\end{aligned}
$$

\section{$\underline{\text { Initial Condition }}$}

Case 1: Deterministic initial condition

As for the univariate equation, the solution of Eq. (3.93) will now be obtained by evaluating $c_{m n}$ from the initial condition. The shearing phase of an unconsol- 
idated undrained triaxial compression test is carried out after the soil specimen is isotropically compressed to a confining pressure, $c$, and hence the initial condition (of Eq. (3.92)) may be represented by a multidimensional Dirac delta function centered at $c$, assuming $c$ is deterministic. Mathematically, this condition can be written as:

$$
P\left(\sigma_{1}, \sigma_{2}, 0\right)=\delta\left(\sigma_{1}-c_{1}\right) \delta\left(\sigma_{2}-c_{2}\right)
$$

or,

$$
P\left(\bar{\sigma}_{1}, \bar{\sigma}_{2}, 0\right)=\delta\left(\bar{\sigma}_{1}-\bar{c}_{1}\right) \delta\left(\bar{\sigma}_{2}-\bar{c}_{2}\right)
$$

where $\bar{c}_{1}=2 \pi\left(c-a_{1}\right) /\left(b_{1}-a_{1}\right)$ and $\bar{c}_{2}=2 \pi\left(c-a_{2}\right) /\left(b_{2}-a_{2}\right)$. Specializing Eq. (3.98) at $t=0$ and equating that with the r.h.s. of Eq. (3.100), the following equation may be obtained:

$$
\delta\left(\bar{\sigma}_{1}-\bar{c}_{1}\right) \delta\left(\bar{\sigma}-\bar{c}_{2}\right)=\sum_{n=-\frac{N}{2}}^{\frac{N}{2}} \sum_{m=-\frac{M}{2}}^{\frac{M}{2}} c_{m n} e^{i\left(n \bar{\sigma}_{1}+m \bar{\sigma}_{2}\right)}
$$

Multiplying both sides by $e^{-i\left(k \bar{\sigma}_{1}+p \bar{\sigma}_{2}\right)}$

$$
e^{-i\left(k \bar{\sigma}_{1}+p \bar{\sigma}_{2}\right)} \delta\left(\bar{\sigma}_{1}-\bar{c}_{1}\right) \delta\left(\bar{\sigma}_{2}-\bar{c}_{2}\right)=\sum_{n=-\frac{N}{2}}^{\frac{N}{2}} \sum_{m=-\frac{M}{2}}^{\frac{M}{2}} c_{m n} e^{i\left((n-k) \bar{\sigma}_{1}+(m-p) \bar{\sigma}_{2}\right)}
$$

Integrating both sides over $[0,2 \pi]$ gives:

$$
\begin{aligned}
& \int_{0}^{2 \pi} \int_{0}^{2 \pi} e^{-i\left(k \bar{\sigma}_{1}+p \bar{\sigma}_{2}\right)} \delta\left(\bar{\sigma}_{1}-\bar{c}_{1}\right) \delta\left(\bar{\sigma}_{2}-\bar{c}_{2}\right) d \bar{\sigma}_{1} d \bar{\sigma}_{2}= \\
& \left.\left.\sum_{n=-\frac{N}{2}}^{\frac{N}{2}} \sum_{m=-\frac{M}{2}}^{\frac{M}{2}} \frac{e^{i\left((n-k) \bar{\sigma}_{1}+(m-p) \bar{\sigma}_{2}\right)}}{(n-k)(m-p)}\right|_{0} ^{2 \pi}\right|_{0} ^{2 \pi}
\end{aligned}
$$


when $n \neq k$ and $m \neq p$ :

$$
\begin{aligned}
& \left.\left.\frac{e^{j\left((n-k) \bar{\sigma}_{1}+(m-p) \bar{\sigma}_{2}\right)}}{(n-k)(m-p)}\right|_{0} ^{2 \pi}\right|_{0} ^{2 \pi}=\frac{e^{j((n-k) \cdot 2 \pi+(m-p) \cdot 2 \pi+(l-q) \cdot 2 \pi)}}{(n-k)(m-p)} \\
& -\frac{1}{(n-k)(m-p)}=\frac{1}{(n-k)(m-p)}-\frac{1}{(n-k)(m-p)}=0
\end{aligned}
$$

But when $n=k$ and $m=p$, Eq. (3.102) becomes:

$$
e^{-i\left(k \bar{\sigma}_{1}+p \bar{\sigma}_{2}\right)} \delta\left(\bar{\sigma}_{1}-\bar{c}_{1}\right) \delta\left(\bar{\sigma}_{2}-\bar{c}_{2}\right)=c_{p k} e^{i\left(0 \bar{\sigma}_{1}+0 \bar{\sigma}_{2}\right)}=c_{p k}
$$

Integrating over $[0,2 \pi]$ gives:

$$
\left.4 \pi^{2} c_{p k}=\int_{0}^{2 \pi} \int_{0}^{2 \pi} e^{-i\left(k \bar{\sigma}_{1}+p \bar{\sigma}_{2}\right)} \delta\left(\bar{\sigma}_{1}-\bar{c}_{1}\right) \delta\left(\bar{\sigma}_{2}-\bar{c}_{2}\right)\right) d \bar{\sigma}_{1} d \bar{\sigma}_{2}=e^{-i\left(k \bar{c}_{1}+p \bar{c}_{2}\right)}
$$

where $d \bar{\sigma}_{1}=\frac{2 \pi}{b_{1}-a_{1}} d \sigma_{1}$ and $d \bar{\sigma}_{2}=\frac{2 \pi}{b_{2}-a_{2}} d \sigma_{2}$. Therefore, the coefficients of the Fourier series are:

$$
c_{p k}=\frac{1}{\left(b_{1}-a_{1}\right)\left(b_{2}-a_{2}\right)} e^{-i\left(k \bar{c}_{1}+p \bar{c}_{2}\right)}
$$

or,

$$
c_{m n}=\frac{1}{\left(b_{1}-a_{1}\right)\left(b_{2}-a_{2}\right)} e^{-i\left(n \bar{c}_{1}+m \bar{c}_{2}\right)}
$$

Hence, the particular solution of Eq. (3.93) can be obtained as:

$$
\begin{aligned}
P\left(\bar{\sigma}_{1}, \bar{\sigma}_{2}, t\right) & =\frac{1}{b_{1}-a_{1}} \frac{1}{b_{2}-a_{2}} . \\
& \sum_{n=-\frac{N}{2}}^{\frac{N}{2}} \sum_{m=-\frac{M}{2}}^{\frac{M}{2}} \exp \left[-\left\{\dot{\epsilon}_{1}\langle\lambda\rangle\left(\frac{2 \pi}{b_{1}-a_{1}}\right) i n t+\dot{\epsilon}_{1}\left\langle-\frac{1}{2} \lambda\right\rangle\left(\frac{2 \pi}{b_{2}-a_{2}}\right) i m t+\right.\right. \\
& \dot{\epsilon}_{1}^{2} \operatorname{Var}[\lambda] \frac{t^{2}}{2}\left(\frac{2 \pi}{b_{1}-a_{1}}\right)^{2} n^{2}+\dot{\epsilon}_{1}^{2} \operatorname{Var}\left[-\frac{1}{2} \lambda\right] \frac{t^{2}}{2}\left(\frac{2 \pi}{b_{2}-a_{2}}\right)^{2} m^{2}+ \\
& 2 \dot{\epsilon}_{1}^{2} \operatorname{Cov}\left[\lambda ;-\frac{1}{2} \lambda\right] \frac{t^{2}}{2}\left(\frac{2 \pi}{b_{1}-a_{1}}\right)\left(\frac{2 \pi}{b_{2}-a_{2}}\right) n m+ \\
& \left.\left.i\left(n \bar{c}_{1}+m \bar{c}_{2}\right)\right\}+i\left(n \bar{\sigma}_{1}+m \bar{\sigma}_{2}\right)\right]
\end{aligned}
$$


Case 2: Probabilistic initial condition

As mentioned earlier, in a real problem, since we have initial uncertainties (e.g., uncertainty in specific gravity of soils in computing the confining pressure), we need to replace the initial condition by a Gaussian function. Therefore, $P(\sigma, 0)$ can be written as:

$$
P\left(\sigma_{1}, \sigma_{2}, 0\right)=\frac{1}{(2 \pi) d_{1} d_{2}} e^{\left[-\frac{1}{2}\left\{\left(\frac{\sigma_{1}-c_{1}}{d_{1}}\right)^{2}+\left(\frac{\sigma_{2}-c_{2}}{d_{2}}\right)^{2}\right\}\right]}
$$

where $\sigma_{r}, c_{r}$ and $d_{r}$ vary from $a_{r}$ to $b_{r}$ and can be written in terms of $\overline{\sigma_{r}}, \overline{c_{r}}$ and $\bar{d}_{r}$ as:

$$
\begin{aligned}
\sigma_{r} & =\frac{b_{r}-a_{r}}{2 \pi} \bar{\sigma}_{r}+a_{r} \\
c_{r} & =\frac{b_{r}-a_{r}}{2 \pi} \bar{c}_{r}+a_{r} \\
d_{r} & =\frac{b_{r}-a_{r}}{2 \pi} \bar{d}_{r}
\end{aligned}
$$

Therefore, by substituting $\sigma_{r}, c_{r}$ and $d_{r}$ in terms of $\bar{\sigma}_{r}, \bar{c}_{r}$ and $\bar{d}_{r}$ into Eq. (3.110)

$$
\begin{aligned}
& P\left(\bar{\sigma}_{1}, \bar{\sigma}_{2}, 0\right)=\frac{1}{(2 \pi)\left(\frac{b_{1}-a_{1}}{2 \pi} \bar{d}_{1}\right)\left(\frac{b_{2}-a_{2}}{2 \pi} \bar{d}_{2}\right)} \\
& \exp \left[-\frac{1}{2}\left\{\left(\frac{\frac{b_{1}-a_{1}}{2 \pi} \bar{\sigma}_{1}+a_{1}-\frac{b_{1}-a_{1}}{2 \pi} \bar{c}_{1}-a_{1}}{\frac{b_{1}-a_{1}}{2 \pi} \bar{d}_{1}}\right)^{2}+\right.\right. \\
& \left.\left(\frac{\frac{b_{2}-a_{2}}{2 \pi} \bar{\sigma}_{2}+a_{2}-\frac{b_{2}-a_{2}}{2 \pi} \bar{c}_{2}-a_{2}}{\frac{b_{2}-a_{2}}{2 \pi} \bar{d}_{2}}\right)\right]
\end{aligned}
$$


Therefore,

$$
\begin{aligned}
& P\left(\bar{\sigma}_{1}, \bar{\sigma}_{2}, 0\right)=\left(\frac{2 \pi}{b_{1}-a_{1}}\right)\left(\frac{2 \pi}{b_{2}-a_{2}}\right) \frac{1}{(2 \pi) \bar{d}_{1} \bar{d}_{2}} \exp \left[-\frac{1}{2}\left\{\left(\frac{\bar{\sigma}_{1}-\bar{c}_{1}}{\bar{d}_{1}}\right)^{2}+\right.\right. \\
& \left.\left.\left(\frac{\bar{\sigma}_{2}-\bar{c}_{2}}{\bar{d}_{2}}\right)^{2}\right\}\right]
\end{aligned}
$$

Specializing Eq. (3.98) at $t=0$ and equating that with the r.h.s. of Eq. (3.115), the following equation may be obtained:

$$
\begin{aligned}
& \left(\frac{2 \pi}{b_{1}-a_{1}}\right)\left(\frac{2 \pi}{b_{2}-a_{2}}\right) \frac{1}{(2 \pi) \bar{d}_{1} \bar{d}_{2}} e^{\frac{-1}{2}\left[\left(\frac{\bar{\sigma}_{1}-\bar{c}_{1}}{d_{1}}\right)^{2}+\left(\frac{\bar{\sigma}_{2}-\bar{c}_{2}}{d_{2}}\right)^{2}\right]} \\
& =\sum_{n=-\frac{N}{2}}^{\frac{N}{2}} \sum_{m=-\frac{M}{2}}^{\frac{M}{2}} e^{i\left((n-k) \bar{\sigma}_{1}+(m-p) \bar{\sigma}_{2}\right)}
\end{aligned}
$$

Multiplying both sides by $e^{-i\left(k \bar{\sigma}_{1}+p \bar{\sigma}_{2}\right)}$

$$
\begin{aligned}
& e^{-i\left(k \bar{\sigma}_{1}+p \bar{\sigma}_{2}\right)}\left(\frac{2 \pi}{b_{1}-a_{1}}\right)\left(\frac{2 \pi}{b_{2}-a_{2}}\right) \frac{1}{(2 \pi) \bar{d}_{1} \bar{d}_{2}} e^{\left[-\frac{1}{2}\left\{\left(\frac{\bar{\sigma}_{1}-\bar{c}_{1}}{d_{1}}\right)^{2}+\left(\frac{\bar{\sigma}_{2}-\bar{c}_{2}}{d_{2}}\right)^{2}\right\}\right]} \\
& =\sum_{n=-\frac{N}{2}}^{\frac{N}{2}} \sum_{m=-\frac{M}{2}}^{\frac{M}{2}} c_{m n} e^{i\left((n-k) \bar{\sigma}_{1}+(m-p) \bar{\sigma}_{2}\right)}
\end{aligned}
$$

Integrating both sides over $[0,2 \pi]$ gives:

$$
\begin{aligned}
& \int_{0}^{2 \pi} \int_{0}^{2 \pi} e^{-i\left(k \bar{\sigma}_{1}+p \bar{\sigma}_{2}\right)}\left(\frac{2 \pi}{b_{1}-a_{1}}\right)\left(\frac{2 \pi}{b_{2}-a_{2}}\right) \frac{1}{(2 \pi) \bar{d}_{1} \bar{d}_{2}} .
\end{aligned}
$$

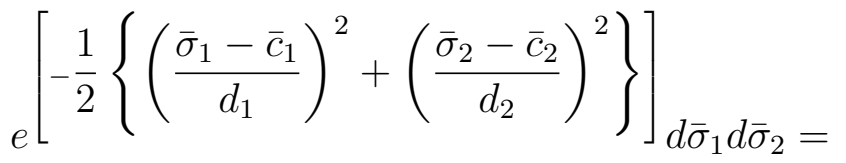

$$
\begin{aligned}
& \left.\left.\sum_{n=-\frac{N}{2}}^{\frac{N}{2}} \sum_{m=-\frac{M}{2}}^{\frac{M}{2}} c_{m n} \frac{e^{i\left((n-k) \bar{\sigma}_{1}+(m-p) \bar{\sigma}_{2}\right)}}{(n-k)(m-p)}\right|_{0} ^{2 \pi}\right|_{0} ^{2 \pi}
\end{aligned}
$$


when $n \neq k$ and $m \neq p$ :

$$
\begin{array}{r}
\left.\left.\frac{e^{j\left((n-k) \bar{\sigma}_{1}+(m-p) \bar{\sigma}_{2}\right)}}{(n-k)(m-p)}\right|_{0} ^{2 \pi}\right|_{0} ^{2 \pi}=\frac{e^{j((n-k) \cdot 2 \pi+(m-p) \cdot 2 \pi}}{(n-k)(m-p)}- \\
\frac{1}{(n-k)(m-p)}=\frac{1}{(n-k)(m-p)}-\frac{1}{(n-k)(m-p)}=0
\end{array}
$$

But when $n=k$ and $m=p$, Eq. (3.117) becomes:

$$
\begin{aligned}
& e^{-i\left(k \bar{\sigma}_{1}+p \bar{\sigma}_{2}\right)}\left(\frac{2 \pi}{b_{1}-a_{1}}\right)\left(\frac{2 \pi}{b_{2}-a_{2}}\right) \frac{1}{(2 \pi) \bar{d}_{1} \bar{d}_{2}} \\
& e^{\left[-\frac{1}{2}\left\{\left(\frac{\bar{\sigma}_{1}-\bar{c}_{1}}{\bar{d}_{1}}\right)^{2}+\left(\frac{\bar{\sigma}_{2}-\bar{c}_{2}}{\bar{d}_{2}}\right)^{2}\right\}\right]}=c_{p k} e^{i\left(0 \bar{\sigma}_{1}+0 \bar{\sigma}_{2}\right)}=c_{p k}
\end{aligned}
$$

Integrating over $[0,2 \pi]$ gives:

$$
\begin{aligned}
& 4 \pi^{2} c_{p k}=\int_{0}^{2 \pi} \int_{0}^{2 \pi}\left[\frac{e^{-i\left(k \bar{\sigma}_{1}+p \bar{\sigma}_{2}\right)}}{(2 \pi) \bar{d}_{1} \bar{d}_{2}}\left(\frac{2 \pi}{b_{1}-a_{1}}\right)\left(\frac{2 \pi}{b_{2}-a_{2}}\right)\right.
\end{aligned}
$$

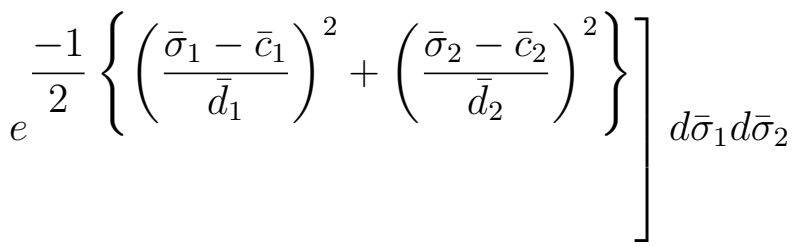

Therefore, $c_{p k}$ can be computed as:

$$
\begin{aligned}
& c_{p k}=\frac{1}{4 \pi^{2}} \int_{0}^{2 \pi} \int_{0}^{2 \pi}\left[\frac{e^{-i\left(k \bar{\sigma}_{1}+p \bar{\sigma}_{2}\right)}}{(2 \pi) \bar{d}_{1} \bar{d}_{2} \bar{d}_{3}}\left(\frac{2 \pi}{b_{1}-a_{1}}\right)\left(\frac{2 \pi}{b_{2}-a_{2}}\right)\right.
\end{aligned}
$$

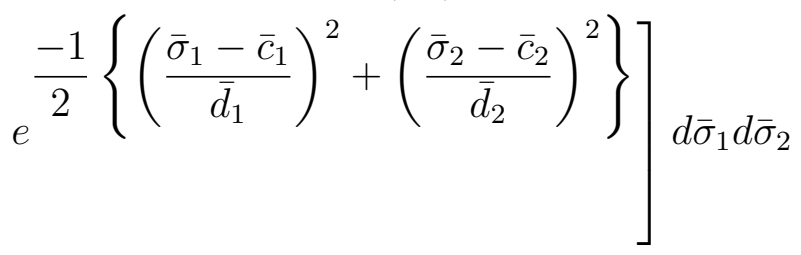


or,

$$
\begin{aligned}
& c_{m n}=\frac{1}{4 \pi^{2}} \int_{0}^{2 \pi} \int_{0}^{2 \pi}\left[\frac{e^{-i\left(k \bar{\sigma}_{1}+p \bar{\sigma}_{2}\right)}}{(2 \pi) \bar{d}_{1} \bar{d}_{2}}\left(\frac{2 \pi}{b_{1}-a_{1}}\right)\left(\frac{2 \pi}{b_{2}-a_{2}}\right)\right. \\
& e^{\left.\frac{-1}{2}\left\{\left(\frac{\bar{\sigma}_{1}-\bar{c}_{1}}{\bar{d}_{1}}\right)^{2}+\left(\frac{\bar{\sigma}_{2}-\bar{c}_{2}}{\bar{d}_{2}}\right)^{2}\right\}\right]} d \bar{\sigma}_{1} d \bar{\sigma}_{2}
\end{aligned}
$$

This integration can be computed analytically (e.g., by using Mathematica or Matlab):

$$
\begin{aligned}
& c_{m n}=\frac{1}{4 \pi^{2}} \frac{1}{\bar{d}_{1} \bar{d}_{2}}\left(\frac{2 \pi}{b_{1}-a_{1}}\right)\left(\frac{2 \pi}{b_{2}-a_{2}}\right) \\
& 0.225079 \exp \left(-\bar{c}_{2} m i-0.5 \bar{d}_{2}^{2} m^{2}-\bar{c}_{1} n i-0.5 \bar{d}_{1}^{2} n^{2}\right) B_{1} B_{2}
\end{aligned}
$$

where

$$
\begin{gathered}
B_{1}=\frac{0.886227 i\left(-6.28319 i+\bar{c}_{2} i+\bar{d}_{2}^{2} m\right) \operatorname{erf}\left[0.707107 \sqrt{-\frac{\left.6.28319 i-\bar{c}_{2} i-\bar{d}_{2}^{2} m\right)^{2}}{\bar{d}_{2}^{2}}}\right]}{\sqrt{-\frac{\left(6.28319 i-\bar{c}_{2} i-\bar{d}_{2}^{2} m\right)^{2}}{\bar{d}_{2}^{2}}}}+ \\
\sqrt{\left(-\frac{\bar{c}_{2}}{\bar{d}_{2}}+\bar{d}_{2} m i\right)^{2}} \\
B_{2}=1.25331 \bar{d}_{1} \operatorname{erf}\left[\frac{0.707107 \bar{c}_{1}}{\bar{d}_{1}}-0.707107 \bar{d}_{1} n i\right]+ \\
1.25331 \bar{d}_{1} \operatorname{erf}\left[\frac{4.44288-0.707107 \bar{c}_{1}+0.707107 \bar{d}_{1}^{2} n i}{\bar{d}_{2}}\right]
\end{gathered}
$$

Solution of Eq. (3.93) can be written as:

$$
\begin{aligned}
& P\left(\bar{\sigma}_{1}, \bar{\sigma}_{2}, 0\right)=\sum_{n=-\frac{N}{2}}^{\frac{N}{2}} \sum_{m=-\frac{M}{2}}^{\frac{M}{2}} c_{m n} \exp \left\{-N_{1_{(1)}}\left(\frac{2 \pi}{b_{1}-a_{1}}\right) i n t-N_{1_{(2)}}\left(\frac{2 \pi}{b_{2}-a_{2}}\right) i m t\right. \\
& -N_{2_{(1)}} \frac{t^{2}}{2}\left(\frac{2 \pi}{b_{1}-a_{1}}\right)^{2} n^{2}-N_{2_{(2)}} \frac{t^{2}}{2}\left(\frac{2 \pi}{b_{2}-a_{2}}\right)^{2} m^{2}- \\
& \left.2 N_{2_{(12)}} \frac{t^{2}}{2}\left(\frac{2 \pi}{b_{1}-a_{1}}\right)\left(\frac{2 \pi}{b_{2}-a_{2}}\right) n m+i\left(n \bar{\sigma}_{1}+m \bar{\sigma}_{2}\right)\right\}
\end{aligned}
$$




\subsubsection{FPK PDEs with Space- and Time-Dependent Coefficients}

FPK PDEs with space- and time-dependent coefficients raise in probabilistic constitutive modeling of elastic-plastic materials. In this subsection, solution algorithm based on Fourier spectral approach for univariate and multivariate forms of the FPK PDEs with space- and but time-dependent coefficients is presented.

The solution algorithm relies on first mapping the stress space of the governing PDE between 0 and $2 \pi$ using the change of coordinates rule, followed by approximating the solution of the PDE in the $2 \pi$-periodic domain by a finite Fourier series in the stress space and unknown time-dependent solution coefficients. Finally, the unknown solution coefficients are approximated using the Euler method.

\section{Univariate Equation}

A univariate FPK PDE with both space- and time-dependent coefficients was obtained in probabilistically modeling von Mises elastic-perfectly plastic soil under unconfined compression test can be written as:

$$
\begin{aligned}
\frac{\partial P(\sigma, t)}{\partial t}= & -\frac{\partial}{\partial \sigma}\left[N_{(1)}^{\sigma^{e q}}(\sigma) P(\sigma, t)\right]+\frac{\partial^{2}}{\partial \sigma^{2}}\left[N_{(2)}^{\sigma^{e q}}(\sigma, t) P(\sigma, t)\right] \\
= & -N_{(1)}^{\sigma^{e q}}(\sigma) \frac{\partial P(\sigma, t)}{\partial \sigma}-P(\sigma, t) \frac{\partial N_{(1)}^{\sigma^{e q}}(\sigma)}{\partial \sigma}+ \\
& N_{(2)}^{\sigma^{e q}}(\sigma, t) \frac{\partial^{2} P(\sigma, t)}{\partial \sigma^{2}}+P(\sigma, t) \frac{\partial^{2} N_{(2)}^{\sigma^{e q}}(\sigma, t)}{\partial \sigma^{2}}+ \\
& 2 \frac{\partial P(\sigma, t)}{\partial \sigma} \frac{\partial N_{(2)}^{\sigma^{e q}}(\sigma, t)}{\partial \sigma}
\end{aligned}
$$

In obtaining the solution of Eq. (3.129), proceeding the same way as for the PDE with time-dependent but space-independent coefficients, Eq. (3.129) can first be re-written in terms of $\bar{\sigma}$ as: 


$$
\begin{aligned}
\frac{\partial P(\bar{\sigma}, t)}{\partial t}= & -N_{(1)}^{\sigma^{e q}}(\sigma) \frac{2 \pi}{b-a} \frac{\partial P(\bar{\sigma}, t)}{\partial \bar{\sigma}}-P(\bar{\sigma}, t) \frac{\partial N_{(1)}^{\sigma^{e q}}(\sigma)}{\partial \sigma}+ \\
& N_{(2)}^{\sigma^{e q}}(\sigma, t)\left(\frac{2 \pi}{b-a}\right)^{2} \frac{\partial^{2} P(\bar{\sigma}, t)}{\partial \bar{\sigma}^{2}}+P(\bar{\sigma}, t) \frac{\partial^{2} N_{(2)}^{\sigma^{e q}}(\sigma, t)}{\partial \sigma^{2}}+ \\
& 2 \frac{2 \pi}{b-a} \frac{\partial P(\bar{\sigma}, t)}{\partial \bar{\sigma}} \frac{\partial N_{(2)}^{\sigma^{e q}}(\sigma, t)}{\partial \sigma}
\end{aligned}
$$

where $N_{(1)}^{\sigma^{e q}}(\sigma)$ and $N_{(2)}^{\sigma^{e q}}(\sigma)$ can be written as

$$
\begin{aligned}
& N_{(1)}^{\sigma^{e q}}(\sigma)=\left(1-P\left[\sigma_{y} \leq \sigma\right]\right) N_{(1)}^{\sigma^{e l}}+P\left[\sigma_{y} \leq \sigma\right] N_{(1)}^{\sigma^{p l^{v M}}} \\
& N_{(2)}^{\sigma^{e q}}(\sigma)=\left(1-P\left[\sigma_{y} \leq \sigma\right]\right) N_{(2)}^{\sigma^{e l}}+P\left[\sigma_{y} \leq \sigma\right] N_{(2)}^{\sigma^{p l^{v M}}}
\end{aligned}
$$

By assuming a normally distributed yield strength $\left(\sigma_{y}\right), P\left[\sigma_{y} \leq \sigma\right]$ will be obtained as:

$$
\begin{aligned}
& P\left[\sigma_{y} \leq \sigma\right]=\mathrm{CDF}\left[\text { Normal Distribution }\left[\left\langle\sigma_{\mathrm{y}}\right\rangle, \sqrt{\operatorname{Var}\left[\sigma_{\mathrm{y}}\right]}\right], \sigma\right]= \\
& \frac{1}{2}\left[1+\operatorname{Erf}\left(\frac{\sigma-\left\langle\sigma_{y}\right\rangle}{\sqrt{2 \operatorname{Var}\left[\sigma_{y}\right]}}\right)\right]
\end{aligned}
$$

Derivatives of $N_{(1)}^{\sigma^{e q}}$ and $N_{(2)}^{\sigma^{e q}}$ can be analytically computed (e.g., by using Mathematica or Matlab) as follows:

$$
\begin{gathered}
\frac{\partial N_{(1)}^{\sigma^{e q}}}{\partial \sigma}=-\frac{e^{-\frac{\left(\sigma-\left\langle\sigma_{y}\right\rangle\right)^{2}}{2 \operatorname{Var}\left[\sigma_{y}\right]}} N_{(1)}^{\sigma^{e l}}}{\sqrt{2 \pi \operatorname{Var}\left[\sigma_{y}\right]}}+\frac{e^{-\frac{\left(\sigma-\left\langle\sigma_{y}\right\rangle\right)^{2}}{2 \operatorname{Var}\left[\sigma_{y}\right]}} N_{(1)}^{\sigma^{p l^{v M}}}}{\sqrt{2 \pi \operatorname{Var}\left[\sigma_{y}\right]}} \\
\frac{\partial N_{(2)}^{\sigma^{e q}}}{\partial \sigma}=-\frac{e^{-\frac{\left(\sigma-\left\langle\sigma_{y}\right\rangle\right)^{2}}{2 \operatorname{Var}\left[\sigma_{y}\right]}} N_{(2)}^{\sigma^{e l}}}{\sqrt{2 \pi \operatorname{Var}\left[\sigma_{y}\right]}}+\frac{e^{-\frac{\left(\sigma-\left\langle\sigma_{y}\right\rangle\right)^{2}}{2 \operatorname{Var}\left[\sigma_{y}\right]}} N_{(2)}^{\sigma^{p l^{v M}}}}{\sqrt{2 \pi \operatorname{Var}\left[\sigma_{y}\right]}}
\end{gathered}
$$




$$
\frac{\partial^{2} N_{(2)}^{\sigma^{e q}}}{\partial \sigma^{2}}=\frac{e^{-\frac{\left(\sigma-\left\langle\sigma_{y}\right\rangle\right)^{2}}{2 \operatorname{Var}\left[\sigma_{y}\right]}} N_{(2)}^{\sigma^{e l}}\left(\sigma-\left\langle\sigma_{y}\right\rangle\right)}{\sqrt{2 \pi \operatorname{Var}\left[\sigma_{y}\right]^{3}}}-\frac{e^{-\frac{\left(\sigma-\left\langle\sigma_{y}\right\rangle\right)^{2}}{2 \operatorname{Var}\left[\sigma_{y}\right]}} N_{(2)}^{\sigma^{p l^{v M}}}\left(\sigma-\left\langle\sigma_{y}\right\rangle\right)}{\sqrt{2 \pi \operatorname{Var}\left[\sigma_{y}\right]^{3}}}
$$

Then, as before, representing the solution of Eq. (3.130) in terms of a finite Fourier series in the $\bar{\sigma}$ space and unknown time-dependent coefficients, $\alpha_{n}$ - i.e., in the form of Eq. (3.58) - it may be written that:

$$
\begin{aligned}
& \sum_{-\frac{N}{2}}^{\frac{N}{2}}\left[\alpha_{n}^{\prime}(t)+\left\{1-\operatorname{Pr}\left[\sigma_{y} \leq \sigma\right]\right\}\langle E\rangle \dot{\epsilon}\left(\frac{2 \pi}{b-a}\right) i n \alpha_{n}(t)+\right. \\
& \langle E\rangle \dot{\epsilon} \frac{\partial}{\partial \sigma}\left\{1-\operatorname{Pr}\left[\sigma_{y} \leq \sigma\right]\right\} \alpha_{n}(t)-t \alpha_{n}(t) \cdot \\
& \left\{\left\{1-\operatorname{Pr}\left[\sigma_{y} \leq \sigma\right]\right\} \operatorname{Var}[E] \dot{\epsilon}^{2}\left(\frac{2 \pi}{b-a}\right)^{2} n^{2}+\right. \\
& 2\left(\frac{2 \pi}{b-a}\right) \operatorname{in} \operatorname{Var}[E] \dot{\epsilon}^{2} \frac{\partial}{\partial \sigma}\left\{1-\operatorname{Pr}\left[\sigma_{y} \leq \sigma\right]\right\}+ \\
& \left.\left.\operatorname{Var}[E] \dot{\epsilon}^{2} \frac{\partial^{2}}{\partial \sigma^{2}}\left\{1-\operatorname{Pr}\left[\sigma_{y} \leq \sigma\right]\right\}\right\}\right] e^{i n \bar{\sigma}}=0
\end{aligned}
$$

Eq. (3.137), in principle, can be integrated to obtain the unknown solution coefficients, $\alpha_{n}$. However, the integration can not be performed analytically. Hence, in the following the Euler method is used to perform the time-integration. To this end, approximating the time derivative, $\alpha_{n}^{\prime}$ by forward difference technique, Eq. (3.137) may be re-written as: 


$$
\begin{aligned}
& \frac{\alpha_{n}\left(t_{j+1}\right)-\alpha_{n}\left(t_{j}\right)}{\Delta t}+\left\{1-\operatorname{Pr}\left[\sigma_{y} \leq \sigma\right]\right\}\langle E\rangle \dot{\epsilon}\left(\frac{2 \pi}{b-a}\right) i n \alpha_{n}\left(t_{j}\right)+ \\
& \langle E\rangle \dot{\epsilon} \frac{\partial}{\partial \sigma}\left\{1-\operatorname{Pr}\left[\sigma_{y} \leq \sigma\right]\right\} \alpha_{n}\left(t_{j}\right)-t_{j+1} \alpha_{n}\left(t_{j}\right) \cdot \\
& \left\{\left\{1-\operatorname{Pr}\left[\sigma_{y} \leq \sigma\right]\right\} \operatorname{Var}[E] \dot{\epsilon}^{2}\left(\frac{2 \pi}{b-a}\right)^{2} n^{2}+\right. \\
& 2\left(\frac{2 \pi}{b-a}\right) i n \operatorname{Var}[E] \dot{\epsilon}^{2} \frac{\partial}{\partial \sigma}\left\{1-\operatorname{Pr}\left[\sigma_{y} \leq \sigma\right]\right\}+ \\
& \left.\operatorname{Var}[E] \dot{\epsilon}^{2} \frac{\partial^{2}}{\partial \sigma^{2}}\left\{1-\operatorname{Pr}\left[\sigma_{y} \leq \sigma\right]\right\}\right\}=0
\end{aligned}
$$

where $\alpha_{n}\left(t_{j+1}\right)$ and $\alpha_{n}\left(t_{j}\right)$ are the values of $\alpha_{n}$ at $t=t_{j}$ and $t=t_{j+1}$, respectively. Rearranging the terms of Eq. (3.138), the following equation may be obtained to compute the values of $\alpha_{n}$ at $t=t_{j+1}$ given the values of $\alpha_{n}$ at $t=t_{j}$ :

$$
\begin{aligned}
\alpha_{n}\left(t_{j+1}\right)=\alpha_{n}\left(t_{j}\right)-\Delta t\left[\left\{1-\operatorname{Pr}\left[\sigma_{y} \leq \sigma\right]\right\}\langle E\rangle \dot{\epsilon}\left(\frac{2 \pi}{b-a}\right) i n \alpha_{n}\left(t_{j}\right)+\right. \\
\langle E\rangle \dot{\epsilon} \frac{\partial}{\partial \sigma}\left\{1-\operatorname{Pr}\left[\sigma_{y} \leq \sigma\right]\right\} \alpha_{n}\left(t_{j}\right)-t_{j+1} \alpha_{n}\left(t_{j}\right) \cdot \\
\quad\left\{\left\{1-\operatorname{Pr}\left[\sigma_{y} \leq \sigma\right]\right\} \operatorname{Var}[E] \dot{\epsilon}^{2}\left(\frac{2 \pi}{b-a}\right)^{2} n^{2}+\right. \\
2\left(\frac{2 \pi}{b-a}\right) i n \operatorname{Var}[E] \dot{\epsilon}^{2} \frac{\partial}{\partial \sigma}\left\{1-\operatorname{Pr}\left[\sigma_{y} \leq \sigma\right]\right\}+ \\
\left.\left.\quad \operatorname{Var}[E] \dot{\epsilon}^{2} \frac{\partial^{2}}{\partial \sigma^{2}}\left\{1-\operatorname{Pr}\left[\sigma_{y} \leq \sigma\right]\right\}\right\}\right]
\end{aligned}
$$

Note that at $t=t_{0}$ the coefficients, $\alpha_{n}\left(t_{0}\right)$ may be computed by comparing Eq. (3.58), specialized at at $t=t_{0}$, with the initial condition, $P\left(\bar{\sigma}_{l}, t_{0}\right)-$ which for an unconfined compression test is given by Eq. (3.65) (or Eq. (3.79) in case of probabilistic initial condition)- as: 


$$
\begin{array}{r}
\sum_{n=-\frac{N}{2}}^{\frac{N}{2}} \alpha_{n}\left(t_{0}\right) e^{i n \bar{\sigma}_{l}}=P\left(\bar{\sigma}_{l}, t_{0}\right) \\
\text { or, } \alpha_{n}\left(t_{0}\right)=\frac{1}{N} \sum_{l=0}^{N} P\left(\bar{\sigma}_{l}, t_{0}\right) e^{-i n \bar{\sigma}_{l}}
\end{array}
$$

where $\bar{\sigma}_{l}=\frac{2 \pi}{N} l$ and $l=0,1,2, \cdots, N$. The coefficients, $\alpha_{n}\left(t_{1}\right)$ at the next time-step, $t=t_{1}$ can then be evaluated using Eq. (3.139) and the solution at $t=t_{1}$ can be computed using Eq. (3.58). The solution can thus be incrementally forwarded in time by updating the coefficients, $\alpha_{n}$ using Eq. (3.139). Note that for stability the product of the number of Fourier terms and (pseudo) time step-size should be less than $2[20,25]$.

$\underline{\text { Multivariate Equation }}$

The multivariate FPK PDE, which was obtained in probabilistically modeling the stress-strain behavior of von Mises elastic-perfectly plastic soil under unconsolidated undrained triaxial compression test, can be written as:

$$
\begin{aligned}
& \frac{\partial P(\boldsymbol{\sigma}, t)}{\partial t}=-\frac{\partial}{\partial \sigma_{1}}\left[N_{(1)_{1}}^{\sigma^{e q}} P(\boldsymbol{\sigma}, t)\right]-\frac{\partial}{\partial \sigma_{2}}\left[N_{(1)_{2}}^{\sigma^{e q}} P(\boldsymbol{\sigma}, t)\right]+ \\
& \frac{\partial^{2}}{\partial \sigma_{1}^{2}}\left[N_{(2)_{11}}^{\sigma^{e q}} P(\boldsymbol{\sigma}, t)\right]+\frac{\partial^{2}}{\partial \sigma_{2}^{2}}\left[N_{(2)_{22}}^{\sigma^{e q}} P(\boldsymbol{\sigma}, t)\right]+2 \frac{\partial^{2}}{\partial \sigma_{1} \partial \sigma_{2}}\left[N_{(2)_{33}}^{\sigma^{e q}} P(\boldsymbol{\sigma}, t)\right]
\end{aligned}
$$


Which can be extended as:

$$
\begin{gathered}
\frac{\partial P(\boldsymbol{\sigma}, t)}{\partial t}=-P(\boldsymbol{\sigma}, t) \frac{\partial N_{(1)_{1}}^{\sigma^{e q}}(\boldsymbol{\sigma})}{\partial \sigma_{1}}-N_{(1)_{1}}^{\sigma^{e q}}(\boldsymbol{\sigma}) \frac{\partial P(\boldsymbol{\sigma}, t)}{\partial \sigma_{1}}-P(\boldsymbol{\sigma}, t) \frac{\partial N_{(1)_{2}}^{\sigma^{e q}}(\boldsymbol{\sigma})}{\partial \sigma_{2}}- \\
N_{(1)_{2}}^{\sigma^{e q}}(\boldsymbol{\sigma}) \frac{\partial P(\boldsymbol{\sigma}, t)}{\partial \sigma_{2}}+N_{(2)_{11}}^{\sigma^{e q}}(\boldsymbol{\sigma}, t) \frac{\partial^{2} P(\boldsymbol{\sigma}, t)}{\partial \sigma_{1}^{2}}+P(\boldsymbol{\sigma}, t) \frac{\partial^{2} N_{(2)_{11}}^{\sigma^{e q}}(\boldsymbol{\sigma}, t)}{\partial \sigma_{1}^{2}}+ \\
2 \frac{\partial N_{(2)_{11}}^{\sigma^{e q}}(\boldsymbol{\sigma}, t)}{\partial \sigma_{1}} \frac{\partial P(\boldsymbol{\sigma}, t)}{\partial \sigma_{1}}+N_{(2)_{22}}^{\sigma^{e q}}(\boldsymbol{\sigma}, t) \frac{\partial^{2} P(\boldsymbol{\sigma}, t)}{\partial \sigma_{2}^{2}}+P(\boldsymbol{\sigma}, t) \frac{\partial^{2} N_{(2)_{22}}^{\sigma^{e q}}(\boldsymbol{\sigma}, t)}{\partial \sigma_{2}^{2}}+ \\
2 \frac{\partial N_{(2)_{22}}^{\sigma^{e q}}(\boldsymbol{\sigma}, t)}{\partial \sigma_{2}} \frac{\partial P(\boldsymbol{\sigma}, t)}{\partial \sigma_{2}}+2 N_{(2)_{12}}^{\sigma^{e q}}(\boldsymbol{\sigma}, t) \frac{\partial^{2} P(\boldsymbol{\sigma}, t)}{\partial \sigma_{1} \partial \sigma_{2}}+2 \frac{\partial N_{(2)_{12}}^{\sigma^{e q}}(\boldsymbol{\sigma}, t)}{\partial \sigma_{1}} \frac{\partial P(\boldsymbol{\sigma}, t)}{\partial \sigma_{2}}+ \\
2 \frac{\partial N_{(2)_{12}}^{\sigma^{e q}}(\boldsymbol{\sigma}, t)}{\partial \sigma_{2}} \frac{\partial P(\boldsymbol{\sigma}, t)}{\partial \sigma_{1}}+2 P(\boldsymbol{\sigma}, t) \frac{\partial^{2} N_{(2)_{12}}^{\sigma^{e q}}(\boldsymbol{\sigma}, t)}{\partial \sigma_{1} \partial \sigma_{2}}
\end{gathered}
$$

where $N_{(1)_{r}}^{\sigma^{e q}}(\sigma)$ and $N_{(2)_{r s}}^{\sigma^{e q}}(\sigma)$ can be written as

$$
\begin{aligned}
& N_{(1)_{r}}^{\sigma^{e q}}(\sigma)=\left(1-P\left[\sigma_{y} \leq \sigma\right]\right) N_{(1)_{r}}^{\sigma^{e l}}+P\left[\sigma_{y} \leq \sigma\right] N_{(1)_{r}}^{\sigma^{p^{v M M}}} \\
& N_{(2)_{r s}}^{\sigma^{e q}}(\sigma)=\left(1-P\left[\sigma_{y} \leq \sigma\right]\right) N_{(2)_{r s}}^{\sigma^{e l}}+P\left[\sigma_{y} \leq \sigma\right] N_{(2)_{r s}}^{\sigma^{p l^{v M}}}
\end{aligned}
$$

In the principal stress space, a von Mises yield surface is a cylinder and can be written as follows [48]:

$$
f=3 J_{2}-k^{2}
$$

where $J_{2}=\frac{1}{2} s_{i j} s_{i j}$ is the second invariant of the deviatoric stress tensor, $s_{i j}=\sigma_{i j}$ $\frac{1}{3} \delta_{i j} \sigma_{k k}$, and $k$ is the yield parameter of the material $\left(k=\sigma_{y}\right)$. Moreover, for the undrained triaxial compression test $\sigma_{2}=\sigma_{3}$. Therefore,

$$
\sigma_{y}^{2}=\frac{\left(\sigma_{1}-\sigma_{2}\right)^{2}+\left(\sigma_{2}-\sigma_{2}\right)^{2}+\left(\sigma_{2}-\sigma_{1}\right)^{2}}{2}=\left(\sigma_{1}-\sigma_{2}\right)^{2}
$$

By assuming a normally distributed yield strength $\left(\sigma_{y}\right), P\left[\sigma_{y} \leq \sigma\right]$ will be obtained as:

$$
\begin{aligned}
& P\left[\sigma_{y} \leq \sigma\right]=\mathrm{CDF}[\text { Normal distribution }[\mu, \sigma], \text { Yield Function }]= \\
& \frac{1}{2}\left(1+\operatorname{erf}\left[\frac{\text { Yield Function }-\left\langle\sigma_{\mathrm{y}}\right\rangle}{\sqrt{2 \operatorname{Var}\left[\sigma_{y}\right]}}\right]\right)
\end{aligned}
$$


On the other hand, if one assumes that $\sigma_{y}$ has a Weibull distribution,

$$
\begin{aligned}
& P\left[\sigma_{y} \leq \sigma\right]=\mathrm{CDF}[\text { Weibull distribution }[\theta, \kappa], \text { Yield Function }]= \\
& 1-\exp \left[-\left(\frac{\text { Yield Function }}{\theta}\right)^{\kappa}\right]
\end{aligned}
$$

where $\theta$ and $\kappa$ are the parameters of the Weibull distribution. They may be obtained from the mean and variance of yield stress using the following relationships [36]:

$$
\begin{aligned}
& \theta \Gamma\left(1+\frac{1}{\kappa}\right)=\left\langle\sigma_{y}\right\rangle \\
& \theta^{2}\left[\Gamma\left(1+\frac{2}{\kappa}\right)-\left\{\Gamma\left(1+\frac{1}{\kappa}\right)\right\}^{2}\right]=\operatorname{Var}\left[\sigma_{y}\right]
\end{aligned}
$$

where $\Gamma(\cdot)$ denotes the gamma function. Note that, derivatives of $N_{1_{(r)}^{e q}}^{e q}$ and $N_{2_{(r s)}^{e q}}^{e q}$ can be analytically computed (e.g., by using Mathematica or Matlab). In order to use the developed algorithm, Eq. (3.142) first is written in terms of $\overline{\boldsymbol{\sigma}}$ as:

$$
\begin{aligned}
& \frac{\partial P(\overline{\boldsymbol{\sigma}}, t)}{\partial t}=-P(\overline{\boldsymbol{\sigma}}, t) \frac{\partial N_{(1)_{1}}^{\sigma^{e q}}(\boldsymbol{\sigma})}{\partial \bar{\sigma}_{1}}-N_{(1)_{1}}^{\sigma^{e q}}(\boldsymbol{\sigma}) \frac{2 \pi}{b_{1}-a_{1}} \frac{\partial P(\overline{\boldsymbol{\sigma}}, t)}{\partial \sigma_{1}}- \\
& P(\overline{\boldsymbol{\sigma}}, t) \frac{\partial N_{(1)_{2}}^{\sigma^{e q}}(\boldsymbol{\sigma})}{\partial \sigma_{2}}-N_{(1)_{2}}^{\sigma^{e q}}(\boldsymbol{\sigma}) \frac{2 \pi}{b_{2}-a_{2}} \frac{\partial P(\overline{\boldsymbol{\sigma}}, t)}{\partial \bar{\sigma}_{2}}+ \\
& \quad N_{(2)_{11}^{\sigma_{1}}}^{e q}(\boldsymbol{\sigma}, t)\left(\frac{2 \pi}{b_{1}-a_{1}}\right)^{2} \frac{\partial^{2} P(\overline{\boldsymbol{\sigma}}, t)}{\partial \bar{\sigma}_{1}^{2}}+P(\overline{\boldsymbol{\sigma}}, t) \frac{\partial^{2} N_{(2)_{11} \sigma_{1}^{e q}}(\boldsymbol{\sigma}, t)}{\partial \sigma_{1}^{2}}+ \\
& 2 \frac{\partial N_{(2)_{11} \sigma_{1}^{e q}}^{2}(\boldsymbol{\sigma}, t)}{\partial \sigma_{1}} \frac{2 \pi}{b_{1}-a_{1}} \frac{\partial P(\overline{\boldsymbol{\sigma}}, t)}{\partial \bar{\sigma}_{1}}+N_{(2)_{22}}^{\sigma^{e q}}(\boldsymbol{\sigma}, t)\left(\frac{2 \pi}{b_{2}-a_{2}}\right)^{2} \frac{\partial^{2} P(\overline{\boldsymbol{\sigma}}, t)}{\partial \bar{\sigma}_{2}^{2}}+ \\
& \quad P(\overline{\boldsymbol{\sigma}}, t) \frac{\partial N_{(2)_{22}}^{2}(\boldsymbol{\sigma}, t)}{\partial \sigma_{2}^{2}}+2 \frac{\partial N_{(2)_{22}}^{\sigma^{e q}}(\boldsymbol{\sigma}, t)}{\partial \sigma_{2}} \frac{2 \pi}{b_{2}-a_{2}} \frac{\partial P(\overline{\boldsymbol{\sigma}}, t)}{\partial \bar{\sigma}_{2}}+ \\
& 2 N_{(2)_{12}}^{\sigma^{e q}}(\boldsymbol{\sigma}, t) \frac{2 \pi}{b_{1}-a_{1}} \frac{2 \pi}{b_{2}-a_{2}} \frac{\partial^{2} P(\overline{\boldsymbol{\sigma}}, t)}{\partial \bar{\sigma}_{1} \partial \bar{\sigma}_{2}}+2 \frac{\partial N_{(2)_{12}}^{\sigma^{e q}}(\boldsymbol{\sigma}, t)}{\partial \sigma_{1}} \frac{2 \pi}{b_{2}-a_{2}} \frac{\partial P(\overline{\boldsymbol{\sigma}}, t)}{\partial \bar{\sigma}_{2}}+ \\
& 2 \frac{\partial N_{(2)_{12}}^{\sigma^{e q}}(\boldsymbol{\sigma}, t)}{\partial \sigma_{2}} \frac{2 \pi}{b_{1}-a_{1}} \frac{\partial P(\overline{\boldsymbol{\sigma}}, t)}{\partial \bar{\sigma}_{1}}+2 P(\overline{\boldsymbol{\sigma}}, t) \frac{\partial^{2} N_{(2)_{12}}^{\sigma^{e q}}(\boldsymbol{\sigma}, t)}{\partial \sigma_{1} \partial \sigma_{2}}
\end{aligned}
$$


Then, as before, representing the solution of Eq. (3.149) in terms of the product of a two-dimensional finite Fourier series in $\overline{\boldsymbol{\sigma}}=\left\{\bar{\sigma}_{1}, \bar{\sigma}_{2}\right\}$ space and unknown time-dependent coefficients, $\alpha_{m n}$ (refer to Eq. (3.94)), a system of ordinary differential equations may be obtained:

$$
\begin{aligned}
& \sum_{n=-\frac{N}{2}}^{\frac{N}{2}} \sum_{m=-\frac{M}{2}}^{\frac{M}{2}}\left[a_{m n}^{\prime}(t)+N_{(1)_{1}}^{e q}\left(\frac{2 \pi}{b_{1}-a_{1}}\right) \text { in } a_{m n}(t)+\frac{\partial N_{(1)_{1}}^{e q}}{\partial \sigma_{1}} a_{m n}(t)+\right. \\
& \left.\left.N_{(1)_{2}}^{e q}\left(\frac{2 \pi}{b_{2}-a_{2}}\right) i m a_{m n}(t)+\frac{\partial N_{()_{2}}^{e q} a_{m n}(t)-}{\partial \sigma_{2}}\right)^{2} n^{2}+2\left(\frac{2 \pi}{b_{1}-a_{1}}\right) i n \frac{\partial N_{(2)_{11}}^{e q}}{\partial \sigma_{1}}+\frac{\partial^{2} N_{(2)_{11}}^{e q}}{\partial \sigma_{1}^{2}}\right\}- \\
& t a_{m n}(t)\left\{-N_{(2)_{11}}^{e q}\left(\frac{2 \pi}{b_{1}-a_{1}}\right)^{2} a_{m n}(t)\left\{-N_{(2)_{22}}^{e q}\left(\frac{2 \pi}{b_{2}-a_{2}}\right)^{2} m^{2}+2\left(\frac{2 \pi}{b_{2}-a_{2}}\right) i m \frac{\partial N_{(2)_{22}}^{e q}}{\partial \sigma_{2}}+\frac{\partial^{2} N_{(2)_{22}}^{e q}}{\partial \sigma_{2}^{2}}\right\}-\right. \\
& 2 t a_{m n}(t)\left\{-N_{(2)_{12}}^{e q}\left(\frac{2 \pi}{b_{1}-a_{1}}\right)\left(\frac{2 \pi}{b_{2}-a_{2}}\right) n m+\left(\frac{2 \pi}{b_{2}-a_{2}}\right) \frac{\partial N_{(2)_{12}}^{e q} i m+}{\partial \sigma_{1}} i m+\right. \\
& \left.\left.\left(\frac{2 \pi}{b_{1}-a_{1}}\right) \frac{\partial N_{(2)_{12}}^{e q}}{\partial \sigma_{2}} i n+\frac{\partial^{2} N_{(2)_{12}}^{e q}}{\partial \sigma_{1} \partial \sigma_{2}}\right\}\right] e^{i\left(n \sigma_{1}+m \sigma_{2}\right)}=0
\end{aligned}
$$

The above equation can be simplified as:

$$
\begin{aligned}
& a_{m n}^{\prime}(t)+N_{(1)_{1}}^{e q}\left(\frac{2 \pi}{b_{1}-a_{1}}\right) \text { in } a_{m n}(t)+\frac{\partial N_{(1)_{1}}^{e q}}{\partial \sigma_{1}} a_{m n}(t)+ \\
& N_{(1)_{2}}^{e q}\left(\frac{2 \pi}{b_{2}-a_{2}}\right) i m a_{m n}(t)+\frac{\partial N_{(1)_{2}}^{e q}}{\partial \sigma_{2}} a_{m n}(t)- \\
& t a_{m n}(t)\left\{-N_{(2)_{11}}^{e q}\left(\frac{2 \pi}{b_{1}-a_{1}}\right)^{2} n^{2}+2\left(\frac{2 \pi}{b_{1}-a_{1}}\right) i n \frac{\partial N_{(2)_{11}}^{e q}}{\partial \sigma_{1}}+\frac{\partial^{2} N_{(2)_{11}}^{e q}}{\partial \sigma_{1}^{2}}\right\}- \\
& t a_{m n}(t)\left\{-N_{(2)_{22}}^{e q}\left(\frac{2 \pi}{b_{2}-a_{2}}\right)^{2} m^{2}+2\left(\frac{2 \pi}{b_{2}-a_{2}}\right) i m \frac{\partial N_{(2)_{22}}^{e q}}{\partial \sigma_{2}}+\frac{\partial^{2} N_{(2)_{22}}^{e q}}{\partial \sigma_{2}^{2}}\right\}- \\
& 2 t a_{m n}(t)\left\{-N_{(2)_{12}}^{e q}\left(\frac{2 \pi}{b_{1}-a_{1}}\right)\left(\frac{2 \pi}{b_{2}-a_{2}}\right) n m+\left(\frac{2 \pi}{b_{2}-a_{2}}\right) \frac{\partial N_{(2)_{12}}^{e q} i m+}{\partial \sigma_{1}} i m+\right. \\
& \left(\frac{2 \pi}{b_{1}-a_{1}}\right) \frac{\left.\partial N_{(2)_{12}}^{e q} i n+\frac{\partial^{2} N_{(2) 1_{2}}^{e q}}{\partial \sigma_{1} \partial \sigma_{2}}\right\}=0}{\partial \sigma_{2}}
\end{aligned}
$$


Finally, employing the Euler method, Eq. (3.151) may be solved for the unknown coefficients, $\alpha_{m n}$, at $t=t_{j+1}$ given the coefficients at $t=t_{j}$ :

$$
\begin{aligned}
& \frac{a_{m n}\left(t_{j+1}\right)-a_{m n}\left(t_{j}\right)}{\Delta t}+N_{(1)_{1}}^{e q}\left(\frac{2 \pi}{b_{1}-a_{1}}\right) i n a_{m n}\left(t_{j}\right)+\frac{\partial N_{(1)_{1}}^{e q}}{\partial \sigma_{1}} a_{m n}\left(t_{j}\right)+ \\
& N_{(1)_{2}}^{e q}\left(\frac{2 \pi}{b_{2}-a_{2}}\right) i m a_{m n}\left(t_{j}\right)+\frac{\partial N_{(1)_{2}}^{e q}}{\partial \sigma_{2}} a_{l m n_{j}}\left(t_{j}\right)- \\
& t_{j} a_{m n}\left(t_{j}\right)\left\{-N_{(2)_{11}}^{e q}\left(\frac{2 \pi}{b_{1}-a_{1}}\right)^{2} n^{2}+2\left(\frac{2 \pi}{b_{1}-a_{1}}\right) i n \frac{\partial N_{(2)_{11}}^{e q}}{\partial \sigma_{1}}+\frac{\partial^{2} N_{(2)_{11}}^{e q}}{\partial \sigma_{1}^{2}}\right\}- \\
& t_{j} a_{m n}\left(t_{j}\right)\left\{-N_{(2)_{22}}^{e q}\left(\frac{2 \pi}{b_{2}-a_{2}}\right)^{2} m^{2}+2\left(\frac{2 \pi}{b_{2}-a_{2}}\right) i m \frac{\partial N_{(2)_{22}}^{e q}}{\partial \sigma_{2}}+\frac{\partial^{2} N_{(2)_{22}}^{e q}}{\partial \sigma_{2}^{2}}\right\}- \\
& 2 t_{j} a_{m n}\left(t_{j}\right)\left\{-N_{(2)_{12}}^{e q}\left(\frac{2 \pi}{b_{1}-a_{1}}\right)\left(\frac{2 \pi}{b_{2}-a_{2}}\right) n m+\left(\frac{2 \pi}{b_{2}-a_{2}}\right) \frac{\partial N_{(2)_{12}}^{e q} i m+}{\partial \sigma_{1}} i m+\right. \\
& \left(\frac{2 \pi}{b_{1}-a_{1}}\right) \frac{\left.\partial N_{(2)_{12}}^{e q} i n+\frac{\partial^{2} N_{(2)_{12}}^{e q}}{\partial \sigma_{2} \partial \sigma_{2}}\right\}=0}{\partial \sigma_{2}}=0
\end{aligned}
$$

or,

$$
\begin{aligned}
& a_{m n}\left(t_{j+1}\right)=a_{m n}\left(t_{j}\right)-\Delta t\left[N_{(1)_{1}}^{e q}\left(\frac{2 \pi}{b_{1}-a_{1}}\right) i n a_{m n}\left(t_{j}\right)+\frac{\partial N_{(1)_{1}}^{e q}}{\partial \sigma_{1}} a_{m n}\left(t_{j}\right)+\right. \\
& N_{(1)_{2}}^{e q}\left(\frac{2 \pi}{b_{2}-a_{2}}\right) i m a_{m n}\left(t_{j}\right)+\frac{\partial N_{(1)_{2}}^{e q} a_{m n}\left(t_{j}\right)-}{\partial \sigma_{2}} \\
& t_{j} a_{m n}\left(t_{j}\right)\left\{-N_{(2)_{11}}^{e q}\left(\frac{2 \pi}{b_{1}-a_{1}}\right)^{2} n^{2}+2\left(\frac{2 \pi}{b_{1}-a_{1}}\right) i n \frac{\partial N_{(2)_{11}}^{e q}}{\partial \sigma_{1}}+\frac{\partial^{2} N_{(2)_{11}}^{e q}}{\partial \sigma_{1}^{2}}\right\}- \\
& t_{j} a_{m n}\left(t_{j}\right)\left\{-N_{(2)_{22}}^{e q}\left(\frac{2 \pi}{b_{2}-a_{2}}\right)^{2} m^{2}+2\left(\frac{2 \pi}{b_{2}-a_{2}}\right) i m \frac{\partial N_{(2)_{22}}^{e q}}{\partial \sigma_{2}}+\frac{\partial^{2} N_{(2)_{22}}^{e q}}{\partial \sigma_{2}^{2}}\right\}- \\
& 2 t_{j} a_{m n}\left(t_{j}\right)\left\{-N_{(2)_{12}}^{e q}\left(\frac{2 \pi}{b_{1}-a_{1}}\right)\left(\frac{2 \pi}{b_{2}-a_{2}}\right) n m+\left(\frac{2 \pi}{b_{2}-a_{2}}\right) \frac{\partial N_{(2)_{12}}^{e q} i m+}{\partial \sigma_{1}} i m\right. \\
& \left.\left(\frac{2 \pi}{b_{1}-a_{1}}\right) \frac{\left.\left.\partial N_{(2)_{12}}^{e q} i n+\frac{\partial^{2} N_{(2)_{12}}^{e q}}{\partial \sigma_{1} \partial \sigma_{2}}\right\}\right]}{\partial \sigma_{2}}\right\}
\end{aligned}
$$

By substituting $N_{(1)_{1}}^{\sigma^{e l}}, N_{(1)_{2}}^{\sigma^{e l}}, N_{(2)_{11}}^{\sigma^{e l}}, N_{(2)_{22}}^{\sigma^{e l}}$, and $N_{(2)_{12}}^{\sigma^{e l}}$ by their definitions (refer to Eqs. (2.32) and (2.29)), the above equation can be written as: 


$$
\begin{aligned}
& \alpha_{m n}\left(t_{j+1}\right)=\alpha_{m n}\left(t_{j}\right)-\Delta t\left[\langle\lambda\rangle \dot{\epsilon}_{1}\left\{1-\operatorname{Pr}\left[\sigma_{y} \leq \sqrt{3 J_{2}}\right]\right\}\left(\frac{2 \pi}{b_{1}-a_{1}}\right) \text { in } \alpha_{m n}\left(t_{j}\right)+\right. \\
& \langle\lambda\rangle \dot{\epsilon}_{1} \frac{\partial}{\partial \sigma_{1}}\left\{1-\operatorname{Pr}\left[\sigma_{y} \leq \sqrt{3 J_{2}}\right]\right\} \alpha_{m n}\left(t_{j}\right)+ \\
& \left\langle-\frac{1}{2} \lambda\right\rangle \dot{\epsilon}_{1}\left\{1-\operatorname{Pr}\left[\sigma_{y} \leq \sqrt{3 J_{2}}\right]\right\}\left(\frac{2 \pi}{b_{2}-a_{2}}\right) i m \alpha_{m n}\left(t_{j}\right)+ \\
& \left\langle-\frac{1}{2} \lambda\right\rangle \dot{\epsilon}_{1} \frac{\partial}{\partial \sigma_{2}}\left\{1-\operatorname{Pr}\left[\sigma_{y} \leq \sqrt{3 J_{2}}\right]\right\} \alpha_{m n}\left(t_{j}\right)- \\
& t_{j+1} \alpha_{m n}\left(t_{j}\right)\left\{\operatorname{Var}[\lambda] \dot{\epsilon}_{1}^{2}\left\{1-\operatorname{Pr}\left[\sigma_{y} \leq \sqrt{3 J_{2}}\right]\right\}\left(\frac{2 \pi}{b_{1}-a_{1}}\right)^{2} n^{2}+\right. \\
& 2\left(\frac{2 \pi}{b_{1}-a_{1}}\right) \operatorname{Var}[\lambda] \dot{\epsilon}_{1}^{2} \frac{\partial}{\partial \sigma_{1}}\left\{1-\operatorname{Pr}\left[\sigma_{y} \leq \sqrt{3 J_{2}}\right]\right\} i n+ \\
& \operatorname{Var}[\lambda] \dot{\epsilon}_{1}^{2} \frac{\partial^{2}}{\partial \sigma_{1}^{2}}\left\{1-\operatorname{Pr}\left[\sigma_{y} \leq \sqrt{3 J_{2}}\right]\right\}+ \\
& \operatorname{Var}\left[-\frac{1}{2} \lambda\right] \dot{\epsilon}_{1}^{2}\left\{1-\operatorname{Pr}\left[\sigma_{y} \leq \sqrt{3 J_{2}}\right]\right\}\left(\frac{2 \pi}{b_{2}-a_{2}}\right)^{2} m^{2}+ \\
& 2\left(\frac{2 \pi}{b_{2}-a_{2}}\right) \operatorname{Var}\left[-\frac{1}{2} \lambda\right] \dot{\epsilon}_{1}^{2} \frac{\partial}{\partial \sigma_{2}}\left\{1-\operatorname{Pr}\left[\sigma_{y} \leq \sqrt{3 J_{2}}\right]\right\} i m+ \\
& \operatorname{Var}\left[-\frac{1}{2} \lambda\right] \dot{\epsilon}_{1}^{2} \frac{\partial^{2}}{\partial \sigma_{2}^{2}}\left\{1-\operatorname{Pr}\left[\sigma_{y} \leq \sqrt{3 J_{2}}\right]\right\}+ \\
& 2 \operatorname{Cov}\left[\lambda ;-\frac{1}{2} \lambda\right] \dot{\epsilon}_{1}^{2}\left\{1-\operatorname{Pr}\left[\sigma_{y} \leq \sqrt{3 J_{2}}\right]\right\}\left(\frac{2 \pi}{b_{1}-a_{1}}\right)\left(\frac{2 \pi}{b_{2}-a_{2}}\right) n m+ \\
& 2\left(\frac{2 \pi}{b_{2}-a_{2}}\right) \operatorname{Cov}\left[\lambda ;-\frac{1}{2} \lambda\right] \dot{\epsilon}_{1}^{2} \frac{\partial}{\partial \sigma_{1}}\left\{1-\operatorname{Pr}\left[\sigma_{y} \leq \sqrt{3 J_{2}}\right]\right\} i m+ \\
& 2\left(\frac{2 \pi}{b_{1}-a_{1}}\right) \operatorname{Cov}\left[\lambda ;-\frac{1}{2} \lambda\right] \dot{\epsilon}_{1}^{2} \frac{\partial}{\partial \sigma_{2}}\left\{1-\operatorname{Pr}\left[\sigma_{y} \leq \sqrt{3 J_{2}}\right]\right\} \text { in }+ \\
& \left.\left.2 \operatorname{Cov}\left[\lambda ;-\frac{1}{2} \lambda\right] \dot{\epsilon}_{1}^{2} \frac{\partial^{2}}{\partial \sigma_{1} \partial \sigma_{2}}\left\{1-\operatorname{Pr}\left[\sigma_{y} \leq \sqrt{3 J_{2}}\right]\right\}\right\}\right]
\end{aligned}
$$

where $\lambda=E /(1+\nu)$. Note that at $t=t_{0}$, the coefficients, $\alpha_{m n}\left(t_{0}\right)$ may be obtained from the initial condition, $P\left(\bar{\sigma}_{l_{1}}, \bar{\sigma}_{l_{2}}, t_{0}\right)$, given by Eq. (3.100), as: 


$$
\begin{array}{r}
\sum_{n=-\frac{N}{2}}^{\frac{N}{2}} \sum_{m=-\frac{M}{2}}^{\frac{M}{2}} \alpha_{m n}\left(t_{0}\right) e^{i\left(n \bar{\sigma}_{l_{1}}+m \bar{\sigma}_{l_{2}}\right)}=P\left(\bar{\sigma}_{l_{1}}, \bar{\sigma}_{l_{2}}, t_{0}\right) \\
\text { or, } \alpha_{m n}\left(t_{0}\right)=\frac{1}{N M} \sum_{l_{1}=0}^{N} \sum_{l_{2}=0}^{M} P\left(\bar{\sigma}_{l_{1}}, \bar{\sigma}_{l_{2}}, t_{0}\right) e^{-i\left(n \bar{\sigma}_{l_{1}}+m \bar{\sigma}_{l_{2}}\right)}
\end{array}
$$

where $\bar{\sigma}_{l_{1}}=\frac{2 \pi}{N} l_{1}, \bar{\sigma}_{l_{2}}=\frac{2 \pi}{M} l_{2}$, and $l_{1}=0,1,2, \cdots, N$, and $l_{2}=0,1,2, \cdots, M$.

Eq. (3.154) may then be used to increment the solution forward in time. 


\section{CHAPTER IV}

\section{SIMULATION OF UNCERTAIN CONSTITUTIVE BEHAVIORS OF SOILS}

In this chapter, the developed algorithms for solving the FPK PDEs with spaceindependent but time-dependent and both space- and time-dependent coefficients that govern the probabilistic constitutive behaviors of linear elastic and elastic-plastic soils, respectively, are used to simulate the uncertain constitutive responses of linear elastic and von Mises elastic-perfectly plastic soils under unconfined compression test and unconsolidated undrained triaxial compression test. The results are shown for both monotonic and cyclic behavior of soils. The accuracy and efficiency of the developed algorithms are also tested.

\subsection{Simulation of Uncertain Monotonic Constitutive Behaviors of Soils}

Simulation results are presented in this section in terms of probabilistic monotonic constitutive responses of linear elastic and elastic-plastic soils under unconfined compression test and unconsolidated undrained triaxial compression test. Note that all the results presented below are processed through a Kaiser window to minimize spurious oscillations in the solutions. The window weights in the Fourier space are computed using the following formula [3]: 


$$
W[n]=\left\{\begin{array}{cc}
\frac{I_{0}\left(\beta \sqrt{1-(2 n / N)^{2}}\right)}{I_{0}(\beta)} & -\frac{N}{2} \leq n \leq \frac{N}{2} \\
0 & \text { otherwise }
\end{array}\right.
$$

where $I_{0}(\cdot)$ denotes the zeroth order modified Bessel function of the first kind, $N$ is the number of Fourier terms, and $\beta$ is an arbitrary, non-negative real number that determines the shape of the window. A value of 10 is used for the parameter, $\beta$.

\subsubsection{Linear Elastic Soil}

\section{Unconfined Compression Simulation}

As discussed in the chapter 2 (section 2.1.1), the univariate FPK PDE that governs the probabilistic stress-strain behavior of linear elastic soils under unconfined compression test can be written as:

$$
\begin{aligned}
\frac{\partial P(\sigma, t)}{\partial t} & =-\frac{\partial}{\partial \sigma}\left[N_{(1)}^{\sigma^{e l}} P(\sigma, t)\right]+\frac{\partial^{2}}{\partial \sigma^{2}}\left[N_{(2)}^{\sigma^{e l}}(t) P(\sigma, t)\right] \\
& =-N_{(1)}^{\sigma^{e l}} \frac{\partial P(\sigma, t)}{\partial \sigma}+N_{(2)}^{\sigma^{e l}}(t) \frac{\partial^{2} P(\sigma, t)}{\partial \sigma^{2}} \\
& =-\dot{\epsilon}\langle E\rangle \frac{\partial P(\sigma, t)}{\partial \sigma}+t \dot{\epsilon}^{2} \operatorname{Var}[E] \frac{\partial^{2} P(\sigma, t)}{\partial \sigma^{2}}
\end{aligned}
$$

Figure 4.1 presents the solution of the above equation. This Figure shows the probability density function of axial stress corresponding to an axial strain of $1 \%$. Note that in this simulation the Young's modulus, $E$, of the soil is assumed to be a normal random variable with a mean value of $100 \mathrm{MPa}$ and a coefficient of variation $(C O V)$ of $20 \%$, and sixty Fourier terms were used. Since the elastic modulus is 
assumed to be normally distributed and the problem is linear, the PDF of stress is normally distributed as well.

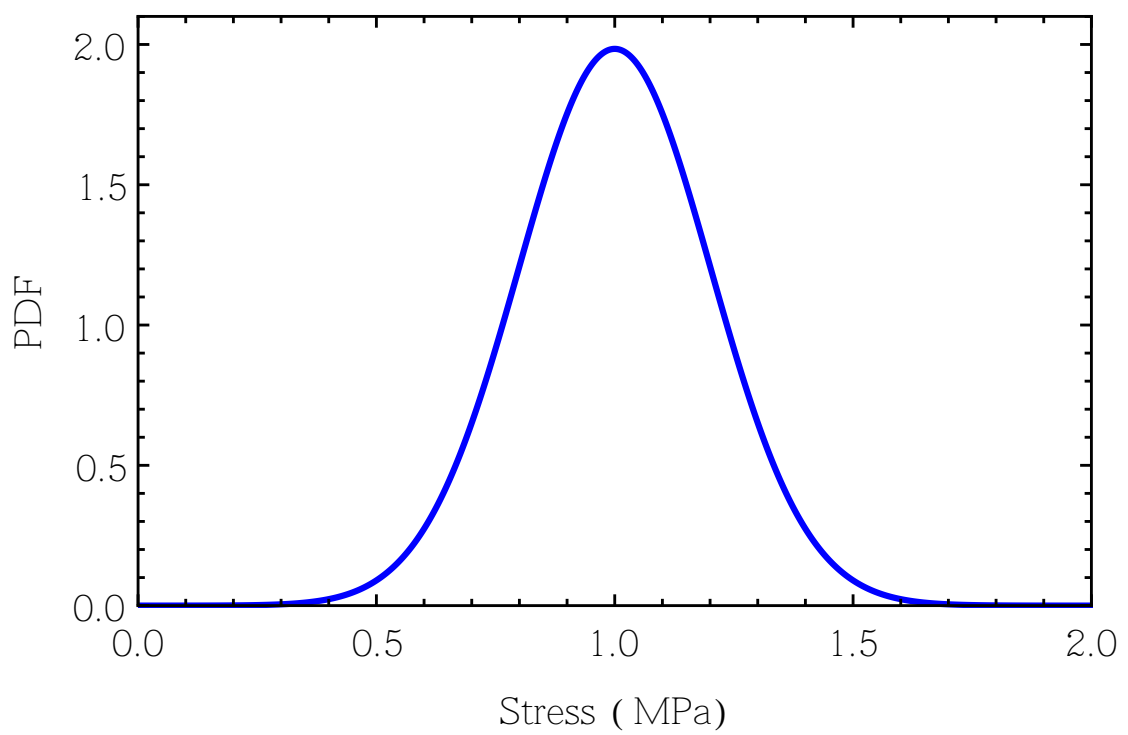

Figure 4.1: Probability density function (PDF) of the axial stress corresponding to an axial strain of $1 \%$ for an uncertain linear elastic soil under an unconfined compression test.

For a linear elastic material, the solutions presented above can also be found, without going through the FPK equations, by simple transformation of variables if the stress-strain relationship (Hooke's law) is written in an algebraic form instead of the rate form. For example, if $\sigma$ and $\epsilon$ are related through the uniaxial Hooke's law, $\sigma=E \epsilon$, where the Young's modulus, $E$, is assumed to be a normal random variable with a mean value of $\langle E\rangle$ and a variance of $\operatorname{Var}[E]$, then the probability density function of $\sigma$ at a given $\epsilon$ may be obtained as: 


$$
g(\sigma)=\frac{1}{\sqrt{2 \pi \operatorname{Var}[\sigma]}} e^{-\frac{(\sigma-\langle\sigma\rangle)^{2}}{2 \operatorname{Var}[\sigma]}}
$$

where,

$$
\begin{gathered}
\langle\sigma\rangle=\langle E\rangle \epsilon+\left\langle\sigma_{c}\right\rangle=100 \times 0.01+0.0=1.0 \mathrm{MPa} \\
\operatorname{Var}[\sigma]=\operatorname{Var}[E] \epsilon^{2}+\operatorname{Var}\left[\sigma_{c}\right]+2 \operatorname{Cov}\left(E, \sigma_{c}\right)= \\
(0.2 \times 100)^{2}(0.01)^{2}+0+0=0.04 \mathrm{MPa}^{2}
\end{gathered}
$$

Note that $\sigma_{c}$ is the initial confining pressure which is assumed to be zero. Eq. (4.5) was used to verify the Fourier spectral solutions presented in Figure 4.1.

The accuracy of the Fourier spectral solutions of the univariate FPK PDEs is presented in Table. 4.1 in terms of average errors in the solution and the number of Fourier terms used to obtain the solution. The average error is computed by subtracting the numerical solution from the actual solution at each stress point and dividing the difference by the maximum value of the actual solution.

The efficiency of the Fourier spectral algorithm is also quantified in terms of the run time. Table 4.1 also compares the accuracy and efficiency of the Fourier spectral solutions with those of the conventional finite difference solutions, which were obtained by numerically approximating both the (stress) space- and time-derivatives using the forward difference technique and solving the resulting system of equations by using the Adam's method.

Table 4.1 shows that, even though, the computational costs increased with the increase in the number of Fourier terms, a small number of Fourier terms were enough 
to obtain reasonable accuracy. This table also shows that the Fourier spectral method is more accurate and more efficient than the conventional finite difference method.

Table 4.1: Comparison of accuracy and efficiency of the Fourier spectral algorithm and conventional finite difference in solving Eq. (4.4) - a univariate FPK PDE with space-independent but time-dependent coefficients.

\begin{tabular}{|c|c|c|c|c|c|}
\hline \multicolumn{3}{|c|}{ Fourier Spectral Method } & \multicolumn{3}{|c|}{ Conventional Finite Difference Method } \\
\hline $\begin{array}{c}\text { Number of } \\
\text { Fourier Terms }\end{array}$ & $\begin{array}{l}\text { Average } \\
\text { Error (\%) }\end{array}$ & $\begin{array}{c}\text { Run Time } \\
(\mathrm{sec})\end{array}$ & $\begin{array}{l}\text { Number of } \\
\text { Grid Points }\end{array}$ & $\begin{array}{l}\text { Average } \\
\text { Error (\%) }\end{array}$ & $\begin{array}{l}\text { Run Time } \\
\text { (min) }\end{array}$ \\
\hline 20 & 0.083 & 0.08 & 5000 & 20 & 20 \\
\hline 50 & 0.013 & 0.17 & 10000 & 1.7 & 161 \\
\hline 100 & 0.003 & 0.32 & 20000 & 0.92 & 1307 \\
\hline 150 & 0.001 & 0.49 & 25000 & 0.52 & 2421 \\
\hline
\end{tabular}

The PDF of the stress (Figure 4.1) contains all the information that is needed for evaluating the stress-strain behavior of the uncertain soil. For instance, statistical moments of the stress (e.g., evolutionary mean and standard deviation) can be found by post-processing this PDF. The evolutionary mean behavior, which is matching with the deterministic solution, shows the ensemble average of behaviors of all the soil particles in a RVE (soil specimen). On the other hand, the evolutionary standard deviation shows the dispersion of the stress values around the mean. Therefore, the most probable values of stress can be visualized as a band around the mean which can be found by computing the evolutionary mean \pm standard deviation. Figure 4.2 
shows the evolutionary marginal mean and mean \pm standard deviation of stress. Note that, in this problem, since the behavior of soil is assumed to be linear elastic, $\mathrm{COV}$ of the stress is equal to the $C O V$ of the elastic modulus $(C O V[\sigma]=C O V[E]=20 \%)$. It should be mentioned that the evolutionary statistics were computed by integrating the solutions - probability density function - after each (pseudo) time step using standard techniques (Eqs. (4.8) and (4.9)).

$$
\begin{array}{lc}
\langle X\rangle=E(X)=\int_{-\infty}^{\infty} x f_{X}(x) d x & \text { for }-\infty<x<\infty \\
\operatorname{Var}[X]=\int_{-\infty}^{\infty}(x-E(X))^{2} f_{X}(x) d x & \text { for }-\infty<x<\infty
\end{array}
$$

where $f_{X}(x)$ is the PDF of random variable $X$.

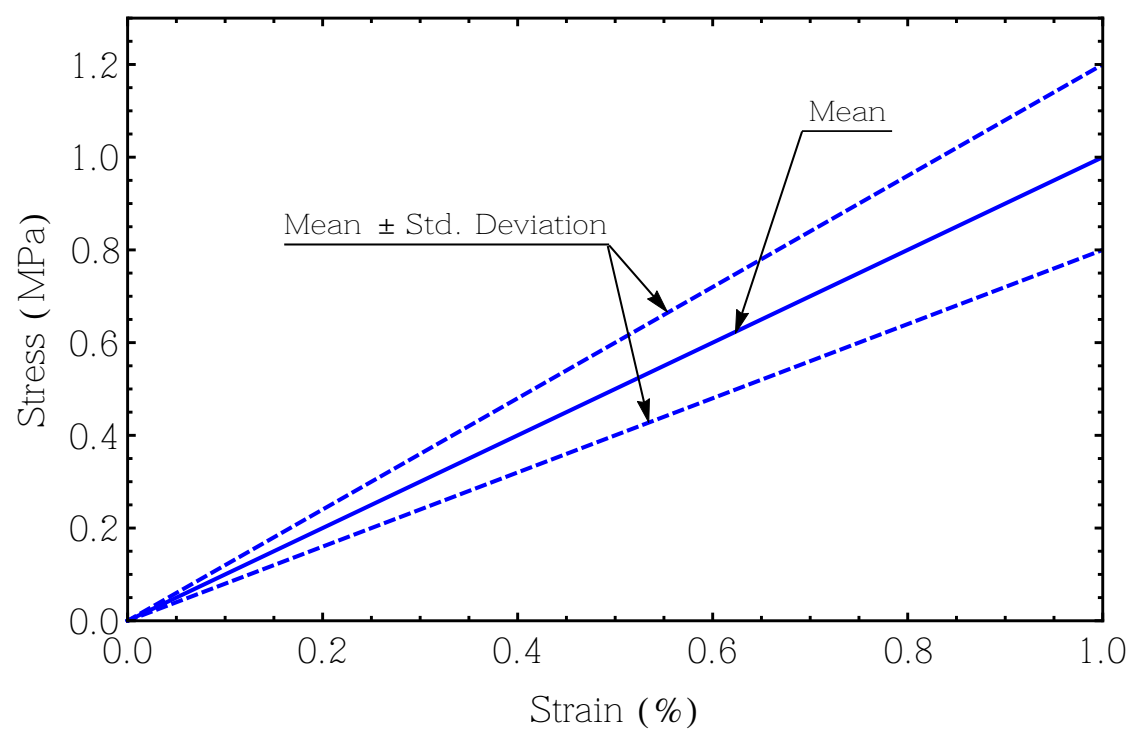

Figure 4.2: Simulated probabilistic elastic behavior in terms evolutionary marginal mean and marginal mean \pm standard deviation behaviors. 


\section{Unconsolidated Undrained Triaxial Compression Simulation}

As discussed in the chapter 2 (section 2.1.2), the bivariate FPK PDE that governs the probabilistic stress-strain behavior of linear elastic soils under unconsolidated undrained triaxial compression test can be written as:

$$
\begin{aligned}
\frac{\partial P\left(\sigma_{1}, \sigma_{2}, t\right)}{\partial t}= & -N_{(1)_{1}}^{\sigma^{e l}} \frac{\partial P\left(\sigma_{1}, \sigma_{2}, t\right)}{\partial \sigma_{1}}-N_{(1)_{2}}^{\sigma^{e l}} \frac{\partial P\left(\sigma_{1}, \sigma_{2}, t\right)}{\partial \sigma_{2}}+ \\
& N_{(2)_{11}}^{\sigma^{e l}}(t) \frac{\partial^{2} P\left(\sigma_{1}, \sigma_{2}, t\right)}{\partial \sigma_{1}^{2}}+N_{(2)_{22}}^{\sigma^{e l}}(t) \frac{\partial^{2} P\left(\sigma_{1}, \sigma_{2}, t\right)}{\partial \sigma_{2}^{2}}+ \\
& 2 N_{(2)_{12}}^{\sigma^{e l}}(t) \frac{\partial^{2} P\left(\sigma_{1}, \sigma_{2}, t\right)}{\partial \sigma_{1} \partial \sigma_{2}}
\end{aligned}
$$

Figure 4.3 presents the solution of the above equation. This Figure shows the joint PDF of $\sigma_{1}$ and $\sigma_{2}$ at $1 \%$ axial strain. This PDF contains all the information on $\sigma_{1}$ and $\sigma_{2}$ and how these two stresses are related at $1 \%$ strain. The evolutionary marginal PDFs of $\sigma_{1}$ and $\sigma_{2}$ can be obtained by integrating the evolutionary joint PDF of $\sigma_{1}$ and $\sigma_{2}$. The evolutionary marginal PDFs of $\sigma_{1}$ and $\sigma_{2}$ can be used to compute the statistical moments (e.g., evolutionary marginal mean and standard deviation) of $\sigma_{1}$ and $\sigma_{2}$. Note that in this simulation, similar to the univariate simulation, the Young's modulus, $E$, of the soil is assumed to be a normal random variable with a mean value of $100 \mathrm{MPa}$ and a coefficient of variation $(C O V)$ of $20 \%$. A deterministic Poisson's ratio of 0.5 and a deterministic initial confining pressure of $50 \mathrm{kPa}$ is assumed, and sixty Fourier terms in each direction were used.

Similar to the univariate case, the solution presented above can also be found, without going through the FPK equation, by simple transformation of variables if the stress-strain relationship (Hooke's law) is written in an algebraic form instead of the 


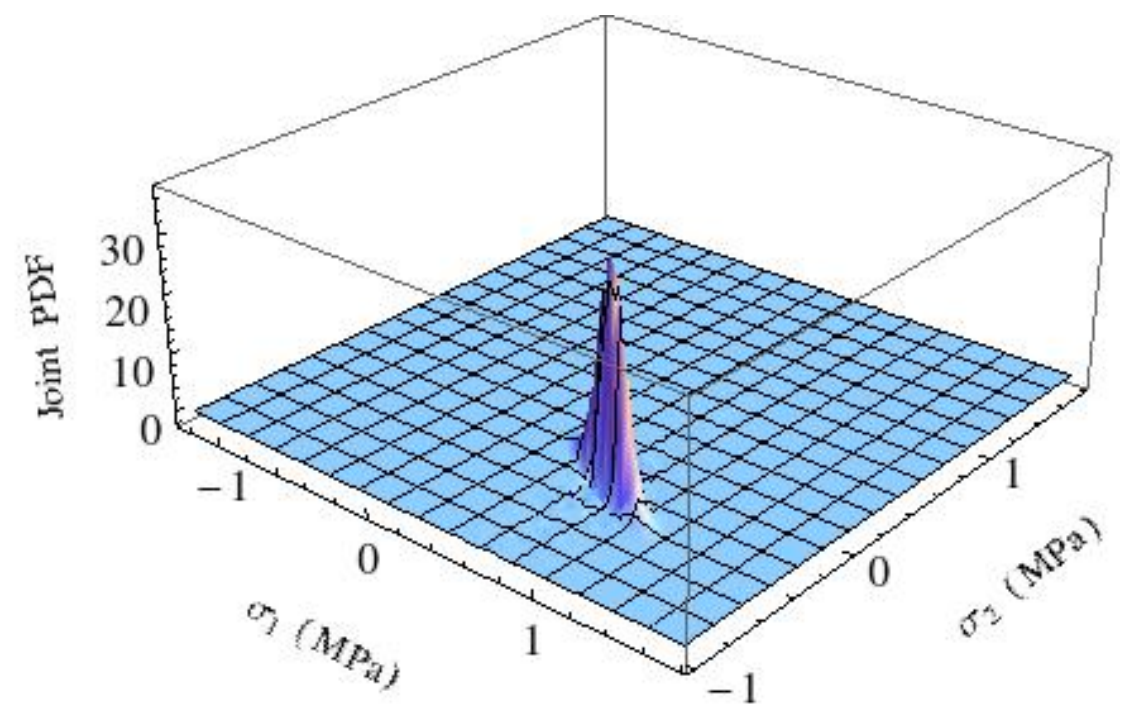

Figure 4.3: Joint probability density function (PDF) of the axial stress $\left(\sigma_{1}\right)$ and radial stress $\left(\sigma_{2}\right)$ at an axial strain of $1 \%$ for an uncertain elastic soil under an unconsolidated undrained triaxial compression test.

rate form. By writing the generalized Hooke's law in an algebraic form, the joint probability density function of axial stress $\left(\sigma_{1}\right)$ and radial stress $\left(\sigma_{2}\right)$ at a given axial strain, $\epsilon_{1}$, may be obtained as:

$$
g\left(\sigma_{1}, \sigma_{2}\right)=\frac{1}{\sqrt{(2 \pi)^{2}|\Sigma|}} e^{-\frac{1}{2}(\boldsymbol{\sigma}-\boldsymbol{\mu})^{T} \boldsymbol{\Sigma}^{-1}(\boldsymbol{\sigma}-\boldsymbol{\mu})}
$$

where $\boldsymbol{\sigma}=\left\{\sigma_{1}, \sigma_{2}\right\}, \boldsymbol{\mu}=\left\{\mu_{1}, \mu_{2}\right\}, \boldsymbol{\Sigma}=\left\{\left\{\operatorname{Var}\left[\sigma_{1}\right], \operatorname{Cov}\left(\sigma_{1}, \sigma_{2}\right)\right\},\left\{\operatorname{Cov}\left(\sigma_{1}, \sigma_{2}\right), \operatorname{Var}\left[\sigma_{2}\right]\right\}\right\}$, and $|\boldsymbol{\Sigma}|$ is the determinant of $\boldsymbol{\Sigma} . \mu_{1}, \mu_{2}, \operatorname{Var}\left[\sigma_{1}\right], \operatorname{Var}\left[\sigma_{2}\right]$, and $\operatorname{Cov}\left(\sigma_{1}, \sigma_{2}\right)$ may be obtained as: 


$$
\begin{aligned}
& \mu_{1}=\left\langle\sigma_{1}\right\rangle=\frac{\langle E\rangle}{(1+\nu)(1-2 \nu)}\left[(1-\nu) \epsilon_{1}+\nu \epsilon_{2}+\nu \epsilon_{2}\right]+\left\langle\sigma_{c}\right\rangle= \\
& \frac{100}{(1+0.5)}(0.01)+0.05=0.716 \\
& \mu_{2}=\left\langle\sigma_{2}\right\rangle=\frac{\langle E\rangle}{(1+\nu)(1-2 \nu)}\left[\nu \epsilon_{1}+(1-\nu) \epsilon_{2}+\nu \epsilon_{2}\right]+\left\langle\sigma_{c}\right\rangle= \\
& \frac{100}{2(1+0.5)}(-0.01)+0.01=-0.283
\end{aligned}
$$

and,

$$
\begin{aligned}
& \operatorname{Var}\left[\sigma_{1}\right]=\operatorname{Var}[E]\left[\frac{1}{(1+\nu)(1-2 \nu)}\left((1-\nu) \epsilon_{1}+\nu \epsilon_{2}+\nu \epsilon_{3}\right)\right]^{2}+\operatorname{Var}\left[\sigma_{c}\right]+ \\
& 2 \operatorname{Cov}\left(E, \sigma_{c}\right)=\frac{20^{2}}{(1+0.5)^{2}}(0.01)^{2}+0+0=0.0177 \\
& \operatorname{Var}\left[\sigma_{2}\right]=\operatorname{Var}[E]\left[\frac{1}{(1+\nu)(1-2 \nu)}\left(\nu \epsilon_{1}+(1-\nu) \epsilon_{2}+\nu \epsilon_{3}\right)\right]^{2}+\operatorname{Var}\left[\sigma_{c}\right]+ \\
& 2 \operatorname{Cov}\left(E, \sigma_{c}\right)=\frac{20^{2}}{(2(1+0.5))^{2}}(0.01)^{2}+0+0=0.0044
\end{aligned}
$$

Since $E$ and $\sigma_{c}$ are independent, the covariance of them $\left(\operatorname{Cov}\left(E, \sigma_{c}\right)\right)$ is zero. The covariance of $\operatorname{Cov}\left(\sigma_{1}, \sigma_{2}\right)$ should also be computed. It should be mentioned that $\operatorname{Cov}(A x, B y)=A B \operatorname{Cov}(x, y)$. Therefore,

$$
\begin{aligned}
& \operatorname{Cov}\left(\sigma_{1}, \sigma_{2}\right)=\left[\frac{1}{(1+\nu)(1-2 \nu)}\left((1-\nu) \epsilon_{1}+\nu \epsilon_{2}+\nu \epsilon_{2}\right)\right] \\
& {\left[\frac{1}{(1+\nu)(1-2 \nu)}\left(\nu \epsilon_{1}+(1-\nu) \epsilon_{2}+\nu \epsilon_{2}\right)\right] \operatorname{Cov}(E, E)}
\end{aligned}
$$

where $\operatorname{Cov}(E, E)=\operatorname{Var}[E]=20^{2}=400$. Therefore, the covariance matrix can be written as:

$$
\Sigma=\left[\begin{array}{cc}
\operatorname{Var}\left[\sigma_{1}\right] & \operatorname{Cov}\left(\sigma_{1}, \sigma_{2}\right) \\
\operatorname{Cov}\left(\sigma_{2}, \sigma_{1}\right) & \operatorname{Var}\left[\sigma_{2}\right]
\end{array}\right]=\left[\begin{array}{cc}
0.0177 & -0.0089 \\
-0.0089 & 0.0044
\end{array}\right]
$$


The accuracy of the Fourier spectral solution of the bivariate FPK PDEs is presented in Table 4.2, in terms of average errors in the solution and the number of Fourier terms used to obtain the solution. The efficiency of the Fourier spectral algorithm is also quantified in terms of the run time. Table 4.2 also compares the accuracy and efficiency of the Fourier spectral solution with those of the conventional finite difference solution, which were obtained by numerically approximating both the (stress) space- and time-derivatives using the forward difference technique and solving the resulting system of equations by using the Adam's method.

Table 4.2: Comparison of accuracy and efficiency of the Fourier spectral algorithm and conventional finite difference in solving Eq. (4.10) - a bivariate FPK PDE with space-independent but time-dependent coefficients.

\begin{tabular}{|c|c|c||c|c|c|}
\hline \hline \multicolumn{2}{|c||}{ Fourier Spectral Method } & \multicolumn{2}{|c|}{ Conventional Finite Difference Method } \\
\hline Number of & Average & Run Time & Number of & Average & Run Time \\
Fourier Terms & Error (\%) & $(\mathrm{sec})$ & Grid Points & Error (\%) & (day) \\
\hline $20 \times 20$ & 0.13 & 5 & $5000 \times 5000$ & 25.2 & 4 \\
\hline $50 \times 50$ & 0.12 & 23 & $10000 \times 10000$ & 2.13 & 32 \\
\hline $100 \times 100$ & 0.10 & 94 & $20000 \times 20000$ & 1.15 & 265 \\
\hline $150 \times 150$ & 0.09 & 204 & $25000 \times 25000$ & 0.65 & 491 \\
\hline \hline
\end{tabular}

Table 4.2 shows that, even though, the computational costs increased with the increase in the number of Fourier terms, a small number of Fourier terms were enough to obtain reasonable accuracy. This table also shows that the Fourier spectral 
method is more accurate and more efficient than the conventional finite difference method. The standard plots of the undrained triaxial compression test can also be found by post-processing the evolutionary joint PDF of $\sigma_{1}$ and $\sigma_{2}$. For instance, the evolutionary marginal mean and mean \pm standard deviation behaviors of deviatoric stress can be obtained by a simple integration; thereby, the response of the soil can be depicted in traditional deviator stress $(q)$ vs. axial strain $\left(\epsilon_{a}\right)$ and deviator stress $(q)$ vs. triaxial shear strain $\left(\epsilon_{q}\right)$ spaces. Figures 4.4(a) and (b) show the results in the above two traditional spaces, but in terms of mean and mean \pm standard deviation behaviors. Note that for the undrained triaxial compression test, triaxial shear strain is equal to the axial strain $\left(\epsilon_{q}=\frac{2}{3}\left(\epsilon_{a}-\epsilon_{q}\right)=\frac{2}{3}\left(\epsilon_{a}+0.5 \epsilon_{a}\right)=\epsilon_{a}\right)$. It should also be mentioned that for a conventional undrained triaxial compression test in which the cell pressure is held constant while the axial stress is increased, one can write [50]:

$$
\delta p=\frac{\delta q}{3}
$$

where $q$ is the deviator stress and $p$ is the volumetric stress. Moreover, the principle of effective stress indicates that the excess pore pressure is the difference between the total and effective volumetric stress which in incremental form can be written as [50]:

$$
\delta u=\delta p-\delta p^{\prime}
$$

Since there is no volume change for an undrained constant volume condition, mean effective stress is constant and $\delta p^{\prime}$ is zero. Therefore,

$$
\delta u=\delta p=\frac{\delta q}{3}
$$


and hence, the evolution of excess pore water pressure, $u$ can also be computed from the evolutionary behavior of deviatoric stress, $q$.

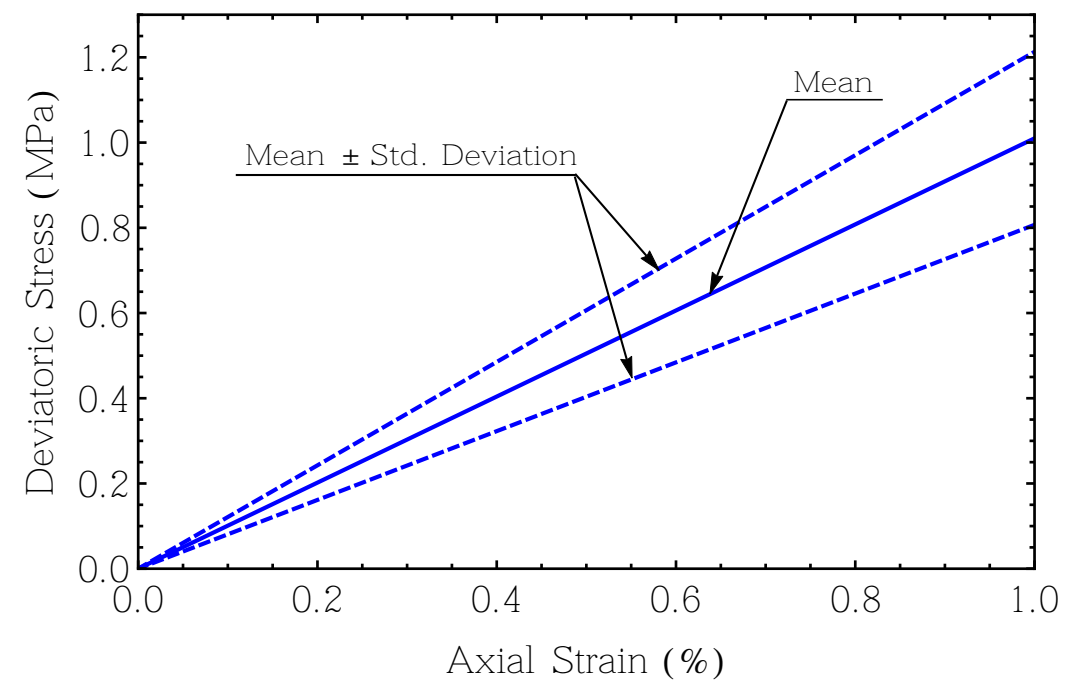

(a)

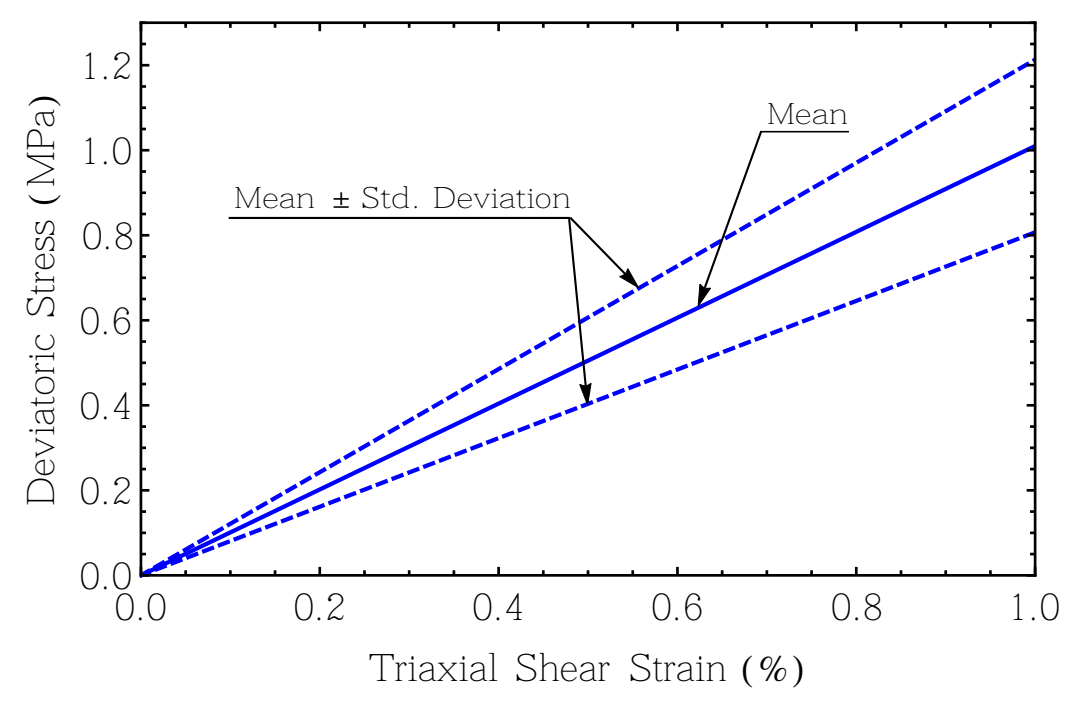

(b)

Figure 4.4: Simulated probabilistic undrained elastic behavior in terms of (a) deviator stress, $q$ versus axial strain, $\epsilon_{a}$, and (b) deviator stress, $q$ versus triaxial shear strain, $\epsilon_{q}$ 
Figure 4.5 shows the evolutionary excess pore water pressure with triaxial shear strain $\left(\epsilon_{q}\right)$ for the uncertain elastic soil. It may be noted that the slopes of $<q>-$ $\epsilon_{a},<\mathrm{q}>-\epsilon_{q}$, and $<\mathrm{u}>-\epsilon_{q}$ were found to be $3 G^{\prime}, 3 G^{\prime}$, and $G^{\prime}\left(G^{\prime}=E /[2(1+\nu)]\right.$, the effective shear modulus), respectively, which agree with the classical soil mechanics theory. This shows that for the linear elastic behavior the mean response is matching with the deterministic solution. However, by performing a probabilistic simulation, mean \pm standard deviation of $q$ and $u$ can also be found. The region between the mean and mean \pm standard deviation depends on the amount of uncertainty that we have in the elastic modulus $(E)$ and shows the most probable values of the response.

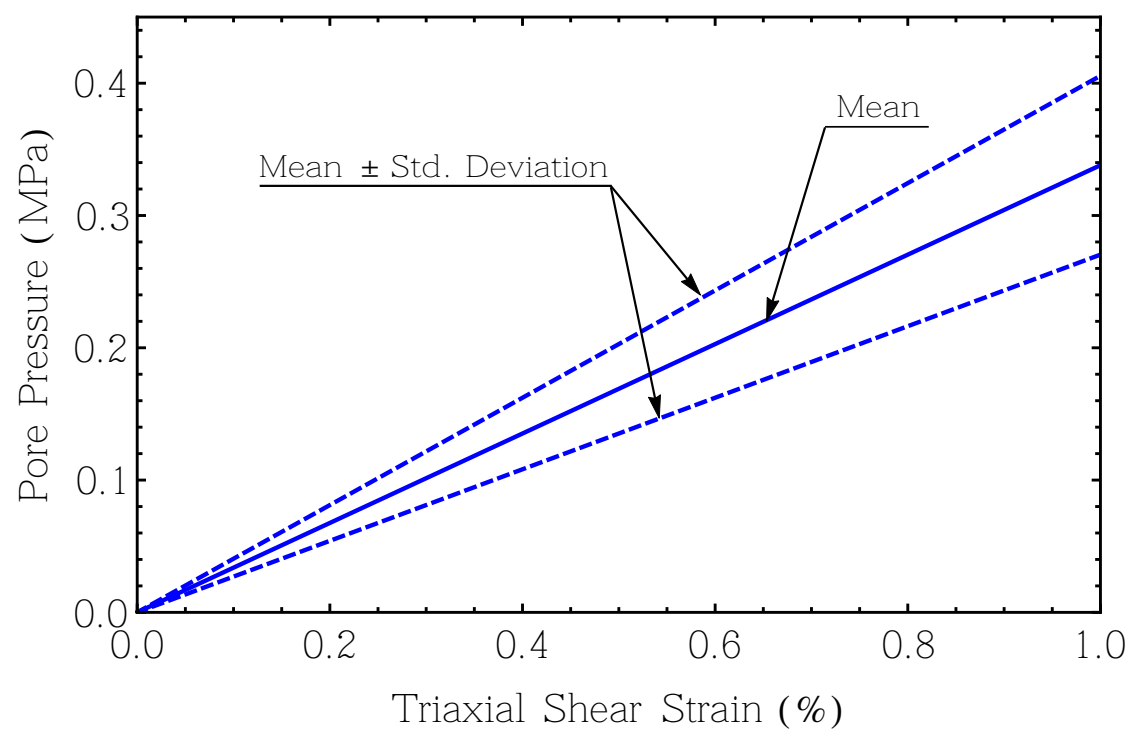

Figure 4.5: Simulated probabilistic undrained elastic behavior in terms of pore pressure, $u$ versus triaxial shear strain, $\epsilon_{q}$. 


\subsection{2 von Mises Elastic-Perfectly Plastic Soil}

\section{Unconfined Compression Simulation}

The univariate FPK PDE that governs the probabilistic stress-strain behavior of von Mises elastic-perfectly plastic soils under unconfined compression test can be written as:

$$
\begin{aligned}
\frac{\partial P(\sigma, t)}{\partial t}= & -\frac{\partial}{\partial \sigma}\left[N_{(1)}^{\sigma^{e q u}}(\sigma) P(\sigma, t)\right]+\frac{\partial^{2}}{\partial \sigma^{2}}\left[N_{(2)}^{\sigma^{e q u}}(\sigma, t) P(\sigma, t)\right] \\
= & -N_{(1)}^{\sigma^{e q u}}(\sigma) \frac{\partial P(\sigma, t)}{\partial \sigma}-P(\sigma, t) \frac{\partial N_{(1)}^{\sigma^{e q}}(\sigma)}{\partial \sigma}+ \\
& N_{(2)}^{\sigma^{e q}}(\sigma, t) \frac{\partial^{2} P(\sigma, t)}{\partial \sigma^{2}}+P(\sigma, t) \frac{\partial^{2} N_{(2)}^{\sigma^{e q}}(\sigma, t)}{\partial \sigma^{2}}+ \\
& 2 \frac{\partial P(\sigma, t)}{\partial \sigma} \frac{\partial N_{(2)}^{\sigma^{e q u}}(\sigma, t)}{\partial \sigma}
\end{aligned}
$$

The solution of the above equation is presented in Figure 4.6. This figure shows the PDF of axial stress corresponding to an axial strain of $1 \%$ for the von Mises elastic-perfectly plastic model. Comparison of this figure with Figure 4.1 shows that for the elastic-perfectly plastic behavior, since the relationship between stress and strain is not linear, the PDF of the stress is not normally distributed anymore. Note that in this simulation, in addition to the soil parameters used for the elastic simulation, an uncertain yield stress $\left(\sigma_{y}\right)$ with a mean of $150 \mathrm{kPa}$ and a $C O V$ of $50 \%$ is assumed. This simulation considered $\sigma_{y}$ to be a normal random variable. Accordingly, the $\sigma$-dependent probabilities appearing in Eq. (3.139) were computed as follows: 


$$
\begin{aligned}
\operatorname{Pr}\left[\sigma_{y} \leq \sigma\right] & =C D F\left[\text { Normal Distribution }\left[\left\langle\sigma_{y}\right\rangle, \sqrt{\operatorname{Var}\left[\sigma_{y}\right]}\right], \sigma\right] \\
& =\frac{1}{2}\left[1+\operatorname{Erf}\left(\frac{\sigma-\left\langle\sigma_{y}\right\rangle}{\sqrt{2 \operatorname{Var}\left[\sigma_{y}\right]}}\right)\right]
\end{aligned}
$$

where $\left\langle\sigma_{y}\right\rangle=150 \mathrm{kPa}, \sqrt{\operatorname{Var}\left[\sigma_{y}\right]}=0.5 \times 150=75 \mathrm{kPa}, C D F[\cdot, \sigma]$ denotes the cumulative density function for the specified distribution evaluated at $\sigma$ and $\operatorname{Erf}(\cdot)$ denotes the error function.

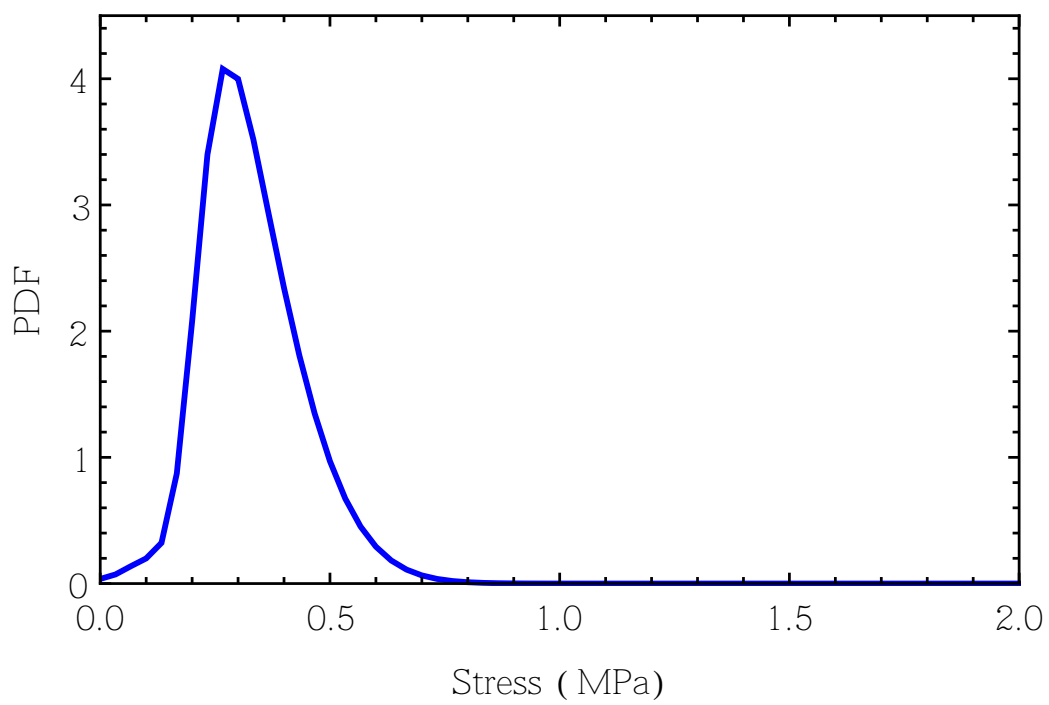

Figure 4.6: Probability density function (PDF) of the axial stress corresponding to an axial strain of $1 \%$ for an uncertain von Mises elastic-perfectly plastic soil under an unconfined compression test.

By post-processing the evolutionary PDF of stress, the statistical moments of the stress (e.g., evolutionary mean and standard deviation), which can be used for the engineering problems, may also be obtained. Figure 4.9 shows the results 
of the elastic-perfectly plastic simulation in terms of the evolutionary mean and mean \pm standard deviation behaviors. This figure shows that, although the material model is elastic-perfectly plastic (i.e, bi-linear), due to uncertainty in yielding, the probabilistic response is smooth, nonlinear and captures the effect of isotropic hardening. This response is very realistic and it means that depending upon the uncertainty in the yield strength, there is always a possibility that the soil becomes elastic-plastic from the very beginning of loading, which agrees with the fact that within a RVE of a spatially non-uniform material like soils some particle-to-particle slips may occur earlier than others. Note that the solutions presented in Figs. 4.6 and 4.7 were obtained using sixty number of Fourier terms.

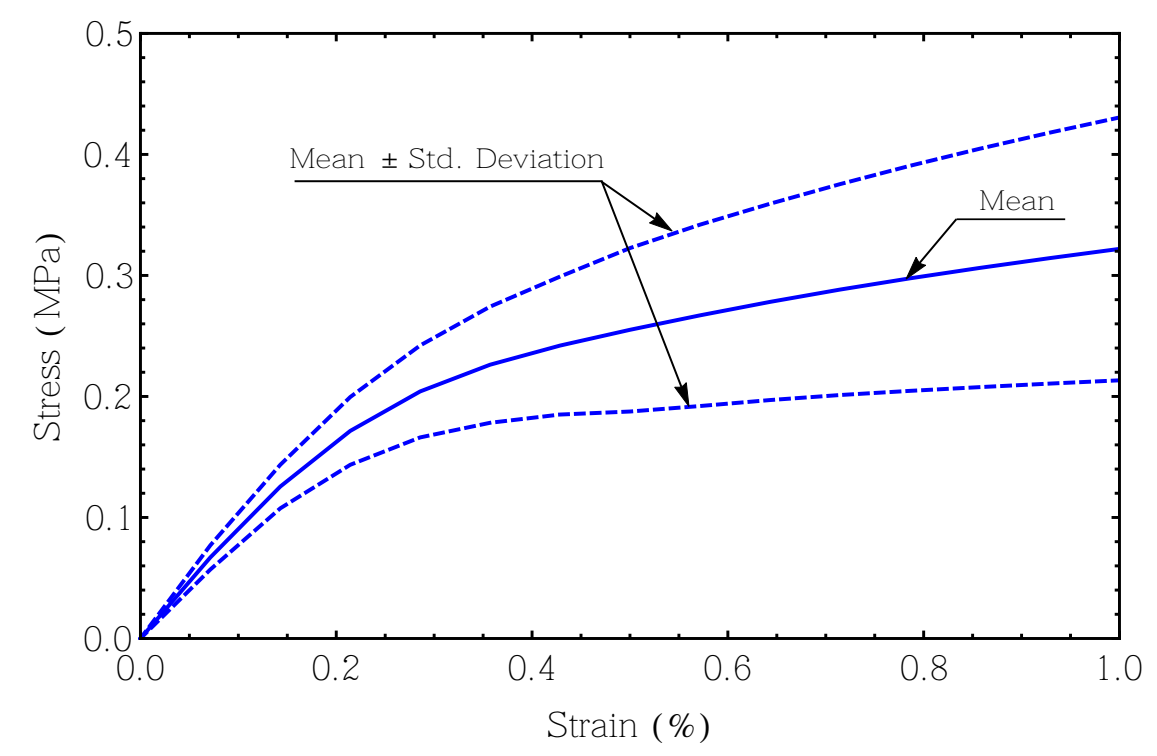

Figure 4.7: Simulated probabilistic elastic-perfectly plastic behavior in terms of the evolutionary mean and mean \pm standard deviation behaviors. 
The effect of the number of Fourier terms on the solutions up to the second order statistics is illustrated in Figure 4.8 in terms of the probability density function of the stress response and in Figure 4.9 in terms of the evolutionary mean and standard deviation behaviors of the stress response with strain, respectively. It can be observed from Figure 4.8 and Figure 4.9 that the PDF of the stress and the evolutionary statistics converge with the increasing number of Fourier terms. Note that, although, by increasing the number of Fourier terms the run time is increasing, a reasonably accurate result can be found by using a small number of Fourier terms (e.g., 100 number of Fourier terms).

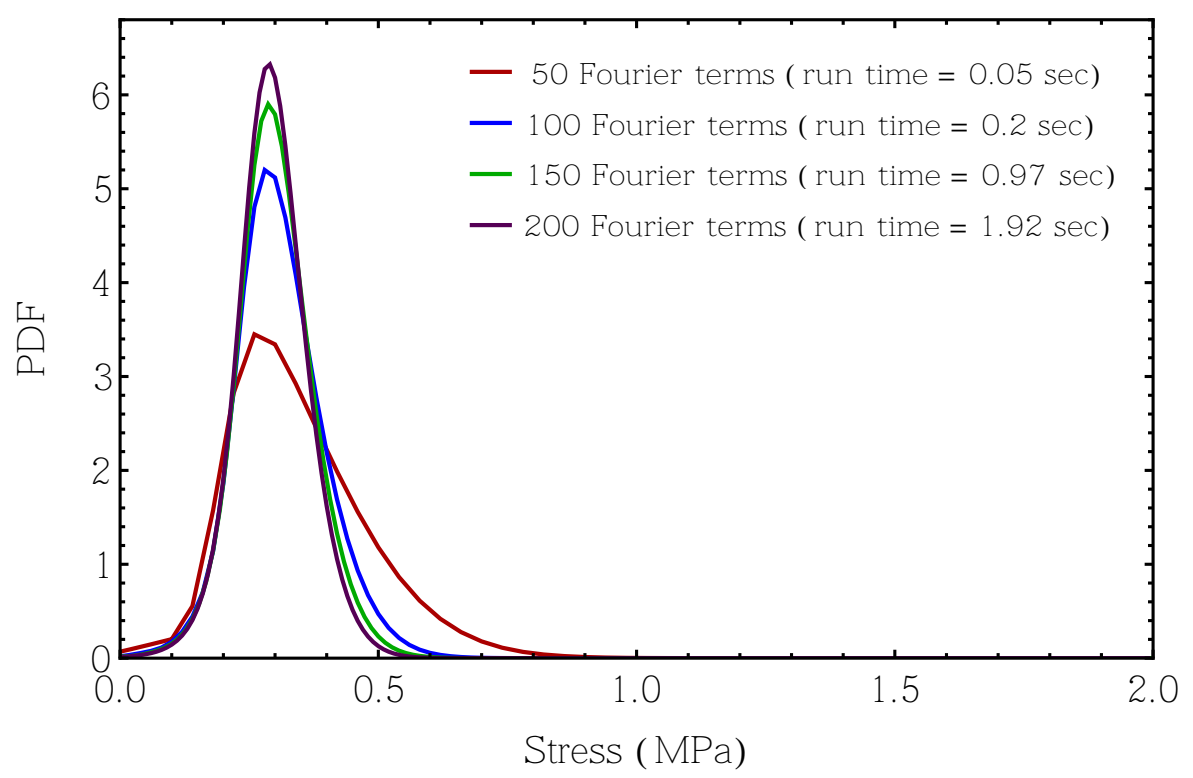

Figure 4.8: Effect of the number of Fourier terms on the Probability density function (PDF) of the axial stress corresponding to an axial strain of $1 \%$ for an uncertain von Mises elastic-perfectly plastic soil under an unconfined compression test. 


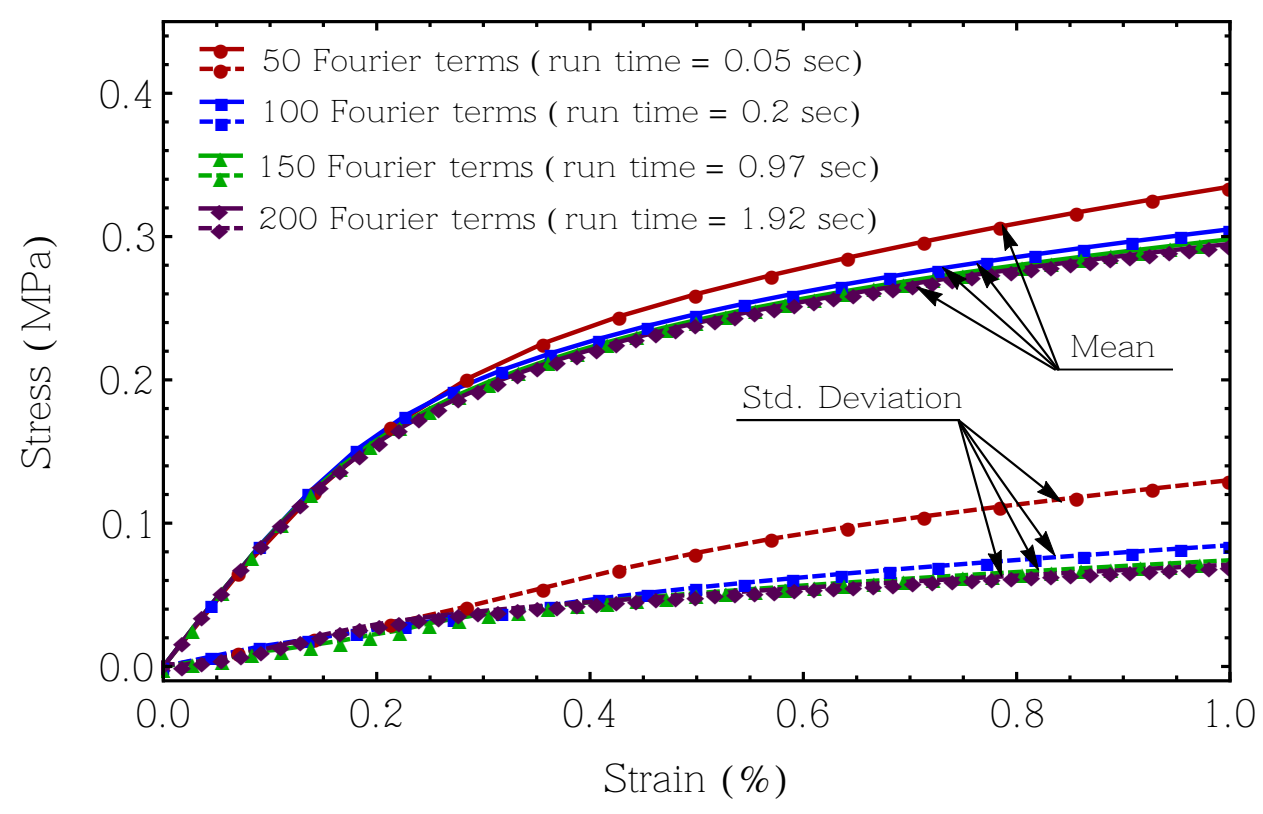

Figure 4.9: Effect of the number of Fourier terms on the simulated evolutionary mean and standard deviation behaviors of the axial stress with the axial strain for an uncertain von Mises elastic-perfectly plastic soil under an unconfined compression test.

\section{Unconsolidated Undrained Triaxial Compression Simulation}

The bivariate FPK PDE that governs the probabilistic stress-strain behavior of von Mises elastic-perfectly plastic soils under unconsolidated undrained triaxial compression test can be written as Eq. (4.24). The solution of this equation, which shows the joint PDF of $\sigma_{1}$ and $\sigma_{2}$, is presented in Figure 4.10. It can be observed that depending upon the uncertainty in the soil parameters, the solution of the FPK equation is advecting and diffusing by going further in time. 


$$
\begin{aligned}
& \frac{\partial P(\boldsymbol{\sigma}, t)}{\partial t}=-P(\boldsymbol{\sigma}, t) \frac{\partial N_{(1)_{1}}^{\sigma^{e q^{v M}}}(\boldsymbol{\sigma})}{\partial \sigma_{1}}-N_{(1)_{1}}^{\sigma^{e q^{v M}}}(\boldsymbol{\sigma}) \frac{\partial P(\boldsymbol{\sigma}, t)}{\partial \sigma_{1}}-P(\boldsymbol{\sigma}, t) \frac{\partial N_{(1)_{2}}^{\sigma^{e q M}}(\boldsymbol{\sigma})}{\partial \sigma_{2}}- \\
& N_{(1)_{2}}^{\sigma^{e q^{v M}}}(\boldsymbol{\sigma}) \frac{\partial P(\boldsymbol{\sigma}, t)}{\partial \sigma_{2}}+N_{(2)_{11}}^{\sigma^{e q^{v M}}}(\boldsymbol{\sigma}, t) \frac{\partial^{2} P(\boldsymbol{\sigma}, t)}{\partial \sigma_{1}^{2}}+P(\boldsymbol{\sigma}, t) \frac{\partial^{2} N_{(2)_{11}^{\sigma^{e v M}}}(\boldsymbol{\sigma}, t)}{\partial \sigma_{1}^{2}}+ \\
& 2 \frac{\partial N_{(2)_{11}^{\sigma^{e q M}}}(\boldsymbol{\sigma}, t)}{\partial \sigma_{1}} \frac{\partial P(\boldsymbol{\sigma}, t)}{\partial \sigma_{1}}+N_{(2)_{22}}^{\sigma^{e q u}}(\boldsymbol{\sigma}, t) \frac{\partial^{2} P(\boldsymbol{\sigma}, t)}{\partial \sigma_{2}^{2}}+P(\boldsymbol{\sigma}, t) \frac{\partial^{2} N_{(2)_{22}}^{\sigma^{e q}}(\boldsymbol{\sigma}, t)}{\partial \sigma_{2}^{2}}+ \\
& 2 \frac{\partial N_{(2)_{22}}^{\sigma^{e q M}}(\boldsymbol{\sigma}, t)}{\partial \sigma_{2}} \frac{\partial P(\boldsymbol{\sigma}, t)}{\partial \sigma_{2}}+2 N_{(2)_{12}}^{\sigma^{e q}}(\boldsymbol{\sigma}, t) \frac{\partial^{2} P(\boldsymbol{\sigma}, t)}{\partial \sigma_{1} \partial \sigma_{2}}+2 \frac{\partial N_{(2)_{12}}^{\sigma^{e q M}}(\boldsymbol{\sigma}, t)}{\partial \sigma_{1}} \frac{\partial P(\boldsymbol{\sigma}, t)}{\partial \sigma_{2}}+ \\
& 2 \frac{\partial N_{(2)_{12}}^{\sigma^{e q}}(\boldsymbol{\sigma}, t)}{\partial \sigma_{2}} \frac{\partial P(\boldsymbol{\sigma}, t)}{\partial \sigma_{1}}+2 P(\boldsymbol{\sigma}, t) \frac{\partial^{2} N_{(2)_{12}}^{\sigma^{e q M}}(\boldsymbol{\sigma}, t)}{\partial \sigma_{1} \partial \sigma_{2}}
\end{aligned}
$$

Note that, the advection of the solution depends on the mean value of the parameters, and the diffusion of the solution, which shows the dispersion around the mean, depends on the standard deviation of the parameters. In other words, if one assumes larger $C O V$ s for the soil parameters, more diffusion will be observed in the joint PDF of the stresses.

In this simulation, in addition to the soil parameters used for the elastic simulation, an uncertain yield stress $\left(\sigma_{y}\right)$ with a mean of $150 \mathrm{kPa}$ and a $C O V$ of $50 \%$ is assumed. This simulation considered $\sigma_{y}$ to be a Weibull random variable and correspondingly, the $\sqrt{J_{2}}$-dependent probabilities appearing in Eq. (3.154) were computed as:

$$
\begin{aligned}
\operatorname{Pr}\left[\sigma_{y} \leq \sqrt{3 J_{2}}\right] & =C D F\left[\text { Weibull Distribution }[\theta, \kappa], \sqrt{3 J_{2}}\right] \\
& = \begin{cases}1-e^{-\left(\frac{\sqrt{3 J_{2}}}{\theta}\right)^{\kappa}} & \text { when } \sqrt{3 J_{2}} \geq 0 \\
0 & \text { when } \sqrt{3 J_{2}}<0\end{cases}
\end{aligned}
$$




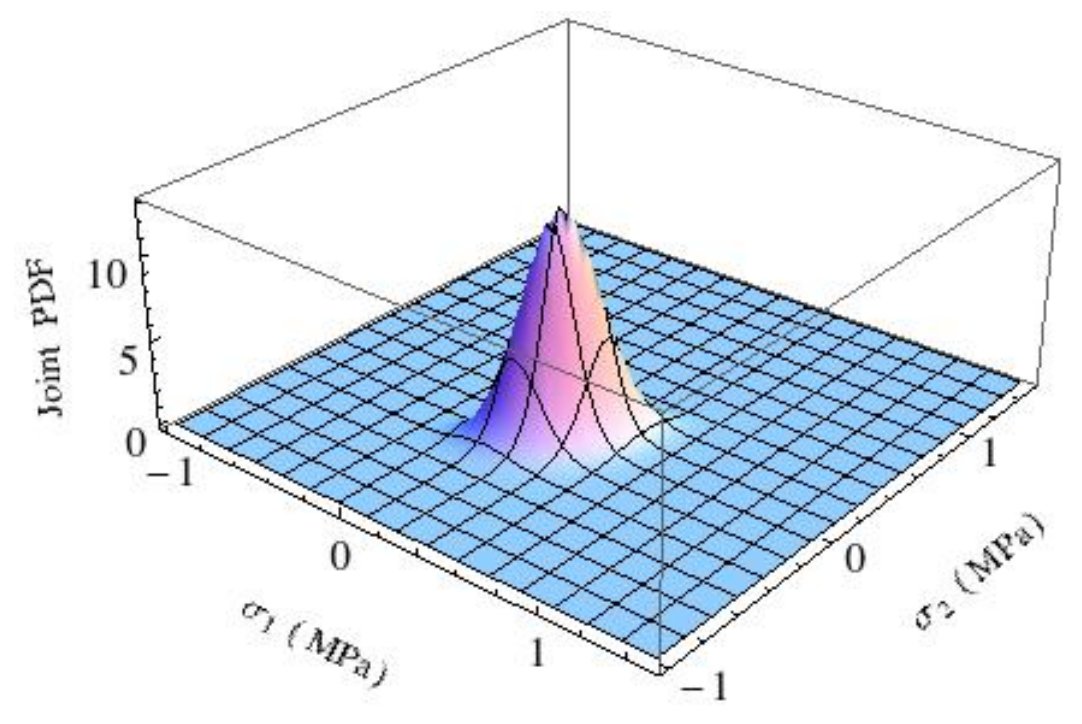

Figure 4.10: Joint probability density function (PDF) of the axial stress $\left(\sigma_{1}\right)$ and radial stress $\left(\sigma_{2}\right)$ at an axial strain of $1 \%$ for an uncertain von Mises elastic-perfectly plastic soil under an unconsolidated undrained triaxial compression test.

where $\theta$ and $\kappa$ are the parameters of the Weibull distribution. They were obtained from the mean and variance of yield stress using the following relationships [36]:

$$
\begin{aligned}
& \theta \Gamma\left(1+\frac{1}{\kappa}\right)=\left\langle\sigma_{y}\right\rangle \\
& \theta^{2}\left[\Gamma\left(1+\frac{2}{\kappa}\right)-\left\{\Gamma\left(1+\frac{1}{\kappa}\right)\right\}^{2}\right]=\operatorname{Var}\left[\sigma_{y}\right]
\end{aligned}
$$

where $\Gamma(\cdot)$ denotes the gamma function.

The marginal PDFs of $\sigma_{1}$ and $\sigma_{2}$ were marginalized by standard integration technique and are shown in Figures 4.11(a) and (b), respectively. These marginal PDFs may be used to obtain all the information (e.g., statistical moments) that one needs for solving the engineering problems. As can be seen in these figures, the marginal PDFs of $\sigma_{1}$ and $\sigma_{2}$ are advecting in different directions which results in 
increasing the evolutionary deviatoric stress $\left(q=\sigma_{1}-\sigma_{2}\right)$. Note that the solutions presented in Figures. 4.10 and 4.11 were obtained using the same numbers of Fourier terms as was used for the elastic simulations. Similar to the linear elastic case, the evolutionary joint PDFs of $\sigma_{1}$ and $\sigma_{2}$ were further processed to compute the corresponding evolutionary marginal mean and standard deviation of deviatoric shear stress; therefore, the response of the soil can be illustrated in traditional deviator stress $(q)$ vs. axial strain $\left(\epsilon_{a}\right)$ and deviator stress $(q)$ vs. triaxial shear strain $\left(\epsilon_{q}\right)$ spaces. The evolution of excess pore water pressure, $u$, can also be computed from the evolutionary behavior of deviatoric stress, $q$. Figures 4.12(a) and (b) and Figure 4.13 show the results in the above three traditional spaces, but in terms of the mean and mean \pm standard deviation behaviors. The mean behavior show that, although the material model is elastic-perfectly plastic (i.e, bi-linear), due to uncertainty in yielding, the probabilistic response is smooth, nonlinear and captures the effect of isotropic hardening. This response is very realistic and it means that depending upon the uncertainty in the yield strength, there is always a possibility that the soil becomes elastic-plastic at very small strains, which agrees with the fact that within a RVE of a spatially non-uniform material like soils some particle-to-particle slips may occur earlier than others. On the other hand, the region between the mean \pm standard deviation shows the most likely values of the deviator stress $(q)$ and the excess pore water pressure $(u)$ with axial strain/triaxial shear strain. Note that, since axial strain $\left(\epsilon_{a}\right)$ and triaxial shear strain $\left(\epsilon_{q}\right)$ in the undrained triaxial compression test are equal, Figures 4.12(a) and (b) are the same. 


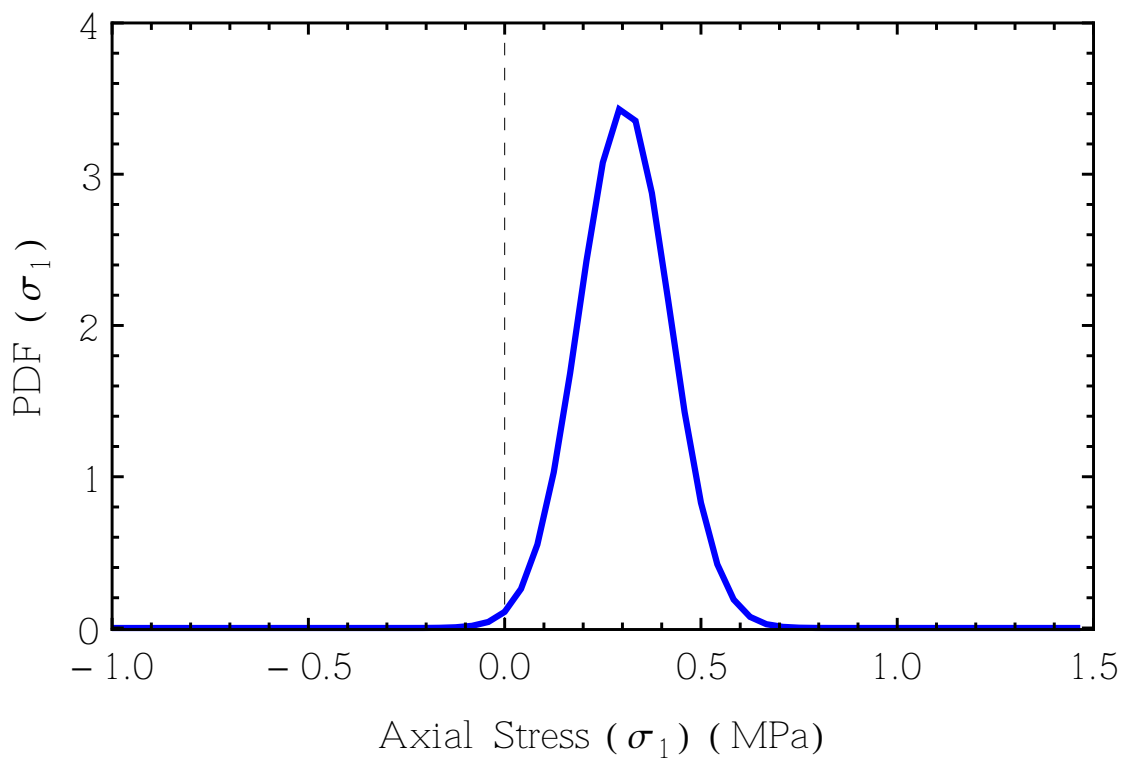

(a)

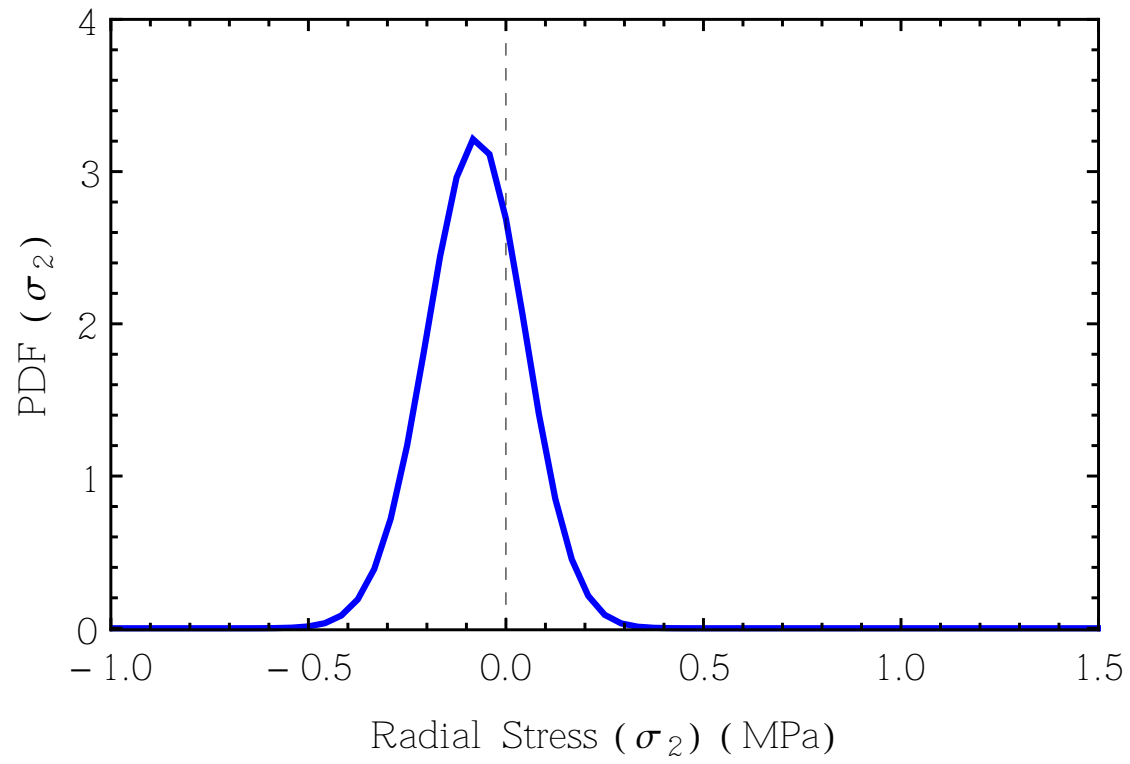

(b)

Figure 4.11: Probability density function (PDF) of the (a) $\sigma_{1}$ corresponding to an axial strain of $1 \%$ and (b) $\sigma_{2}$ corresponding to an axial strain of $1 \%$. 


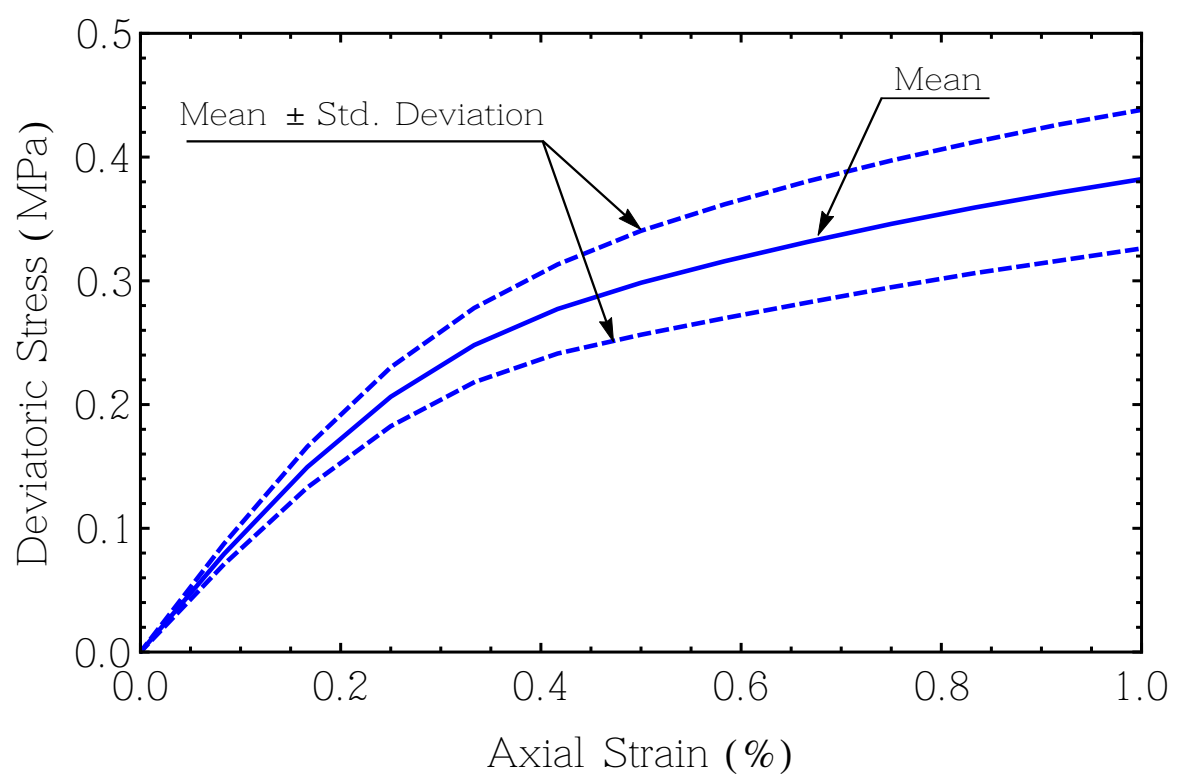

(a)

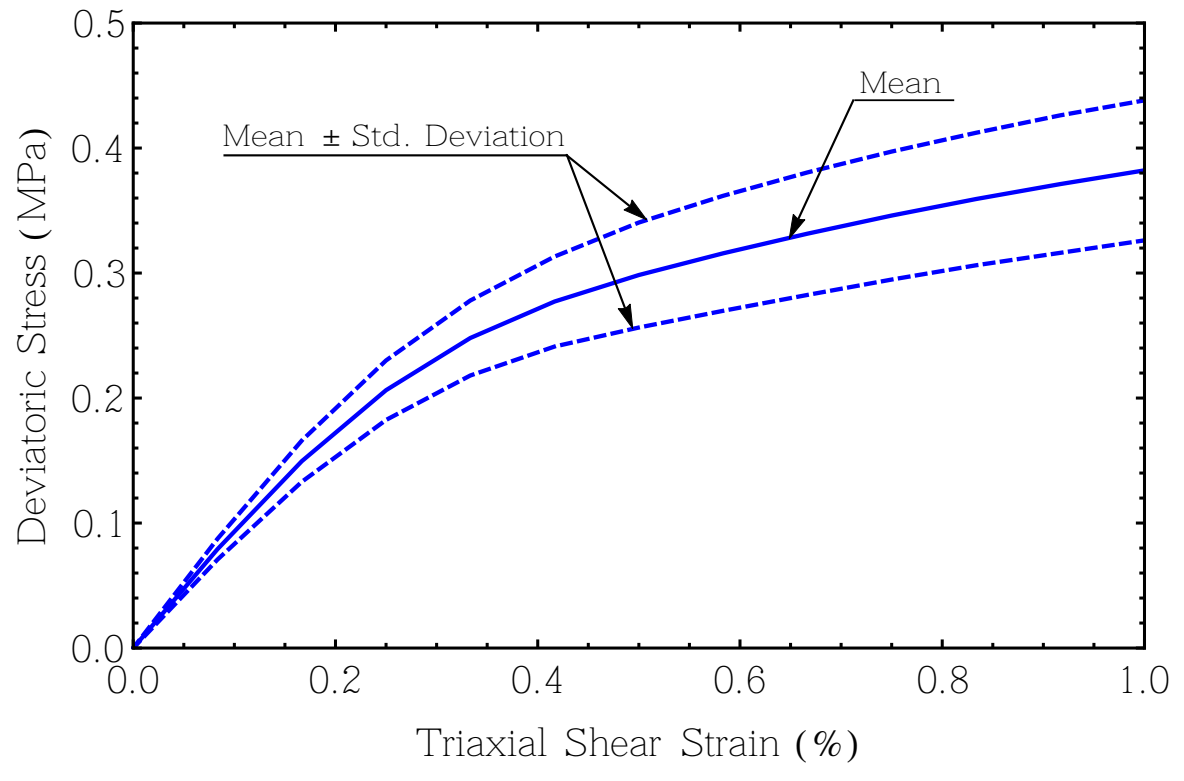

(b)

Figure 4.12: Simulated probabilistic undrained elastic-perfectly plastic behavior in terms of the (a) deviator stress, $q$ versus axial strain, $\epsilon_{a}$ and (b) deviator stress, $q$ versus triaxial shear strain, $\epsilon_{q}$. 


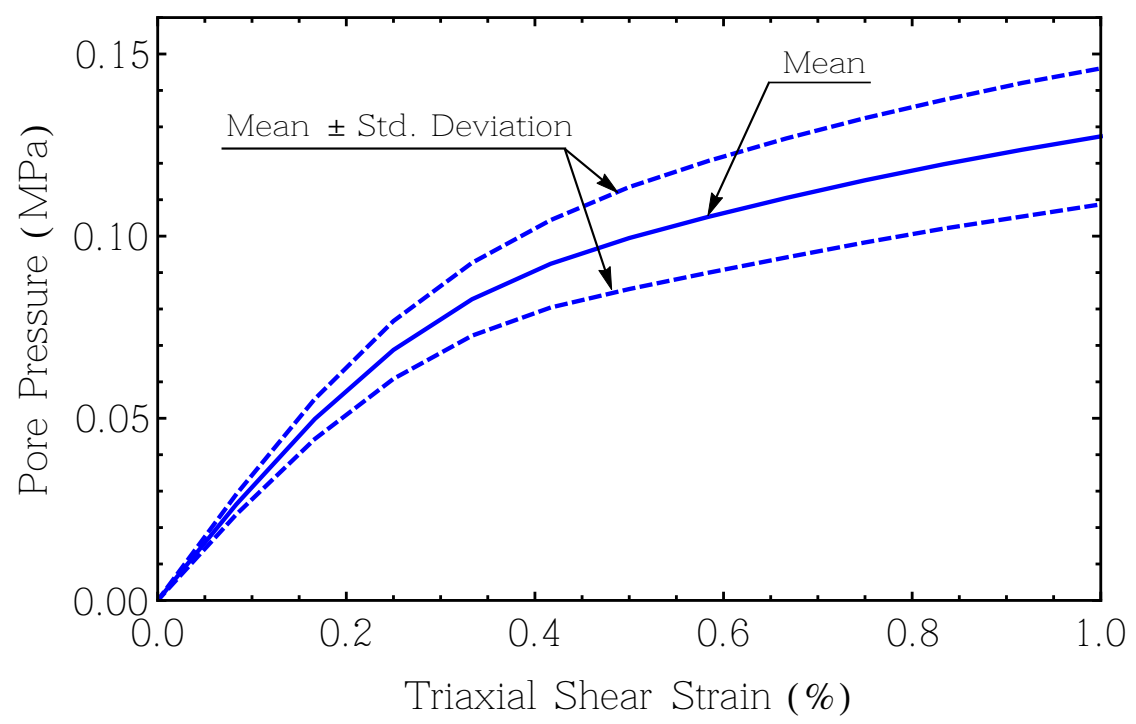

Figure 4.13: Simulated probabilistic undrained elastic-perfectly plastic behavior in terms of the pore pressure, $u$ versus triaxial shear strain, $\epsilon_{q}$.

The effect of the number of Fourier terms on the solutions up to the second order statistics is illustrated in Figure 4.14 to Figure 4.17 in terms of the evolutionary marginal mean and marginal standard deviation behaviors of the components of the stresses, the evolutionary covariance between the stress components with strain, and the evolutionary correlation coefficient of the stress components with strain. It can be observed from Figure 4.14 to Figure 4.17 that, even though, the computational costs could significantly increase with the number of Fourier terms, only a small number of Fourier terms are enough to obtain reasonable accuracies.

It is important to note that the covariance of $\sigma_{1}$ and $\sigma_{2}$ shows how much $\sigma_{1}$ and $\sigma_{2}$ change together (Eq. (4.27)). In general, the sign of the covariance of two variables shows the tendency in the linear relationship between the variables. On 
the other hand, the magnitude of the covariance is not easy to interpret. However, the magnitude of the normalized version of the covariance, the correlation coefficient $\left(\rho_{\sigma_{1}, \sigma_{2}}\right)$, shows the strength of the linear relation (Eq. (4.28)).

$$
\begin{aligned}
\operatorname{cov}\left(\sigma_{1}, \sigma_{2}\right) & =E\left[\sigma_{1} \sigma_{2}\right]-E\left[\sigma_{1}\right] E\left[\sigma_{2}\right] \\
\rho_{\sigma_{1}, \sigma_{2}} & =\frac{\operatorname{Cov}\left(\sigma_{1}, \sigma_{2}\right)}{\sqrt{\operatorname{Var}\left[\sigma_{1}\right] \operatorname{Var}\left[\sigma_{2}\right]}}
\end{aligned}
$$

Figure 4.17 shows the effect of the number of Fourier terms on the evolutionary correlation coefficient of $\sigma_{1}$ and $\sigma_{2}$. Note that, in this problem, since $\sigma_{1}$ and $\sigma_{2}$ show opposite behavior (PDF of $\sigma_{1}$ is moving to the right direction, and PDF of $\sigma_{2}$ is moving to the left direction), $\sigma_{1}$ and $\sigma_{2}$ are negatively correlated.

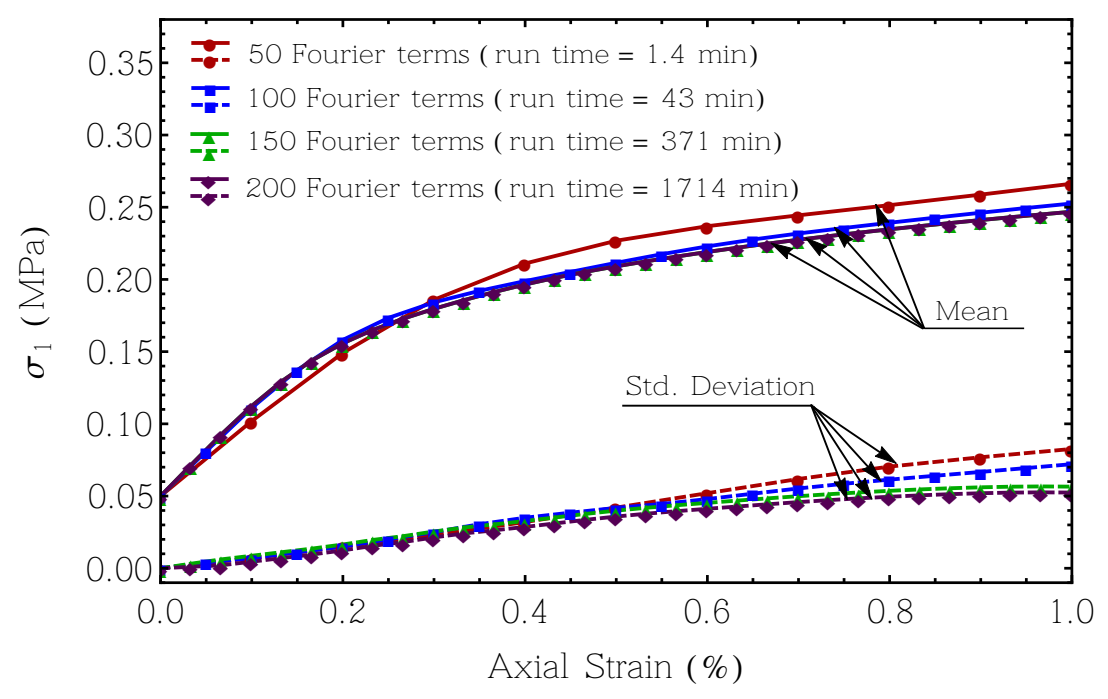

Figure 4.14: Effect of the number of Fourier terms on the simulated evolutionary marginal mean and marginal standard deviation of the axial stress for an uncertain von Mises elastic-perfectly plastic soil under an unconsolidated undrained triaxial compression test. 


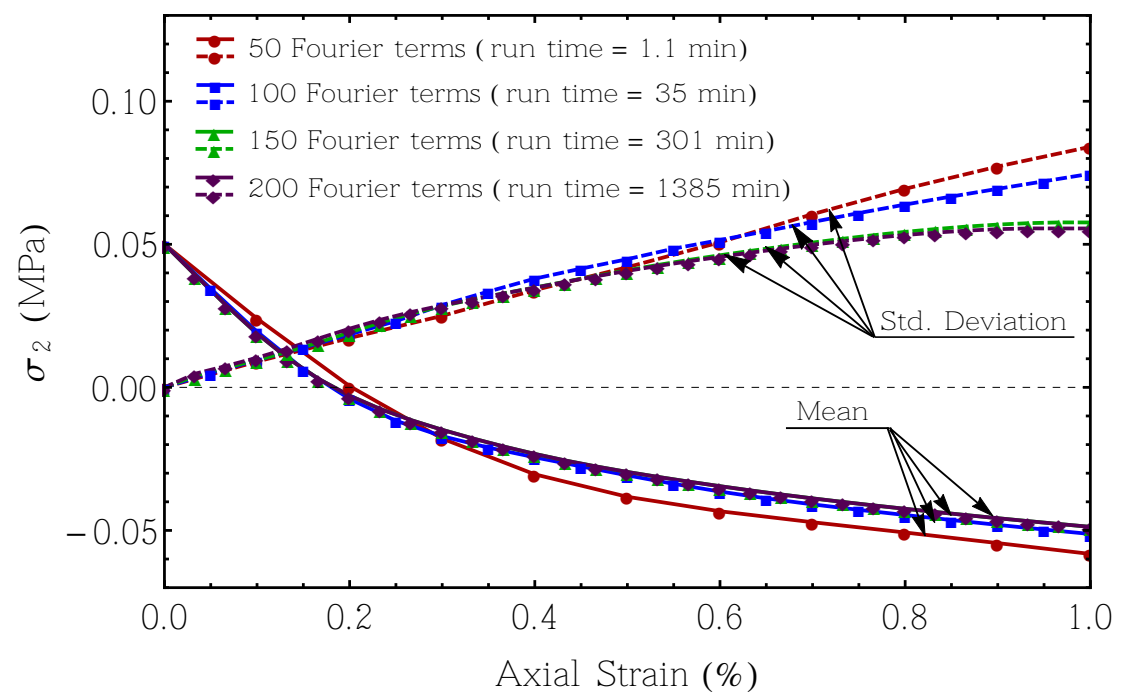

Figure 4.15: Effect of the number of Fourier terms on the simulated evolutionary marginal mean and marginal standard deviation of the radial stress for an uncertain von Mises elastic-perfectly plastic soil under an unconsolidated undrained triaxial compression test.

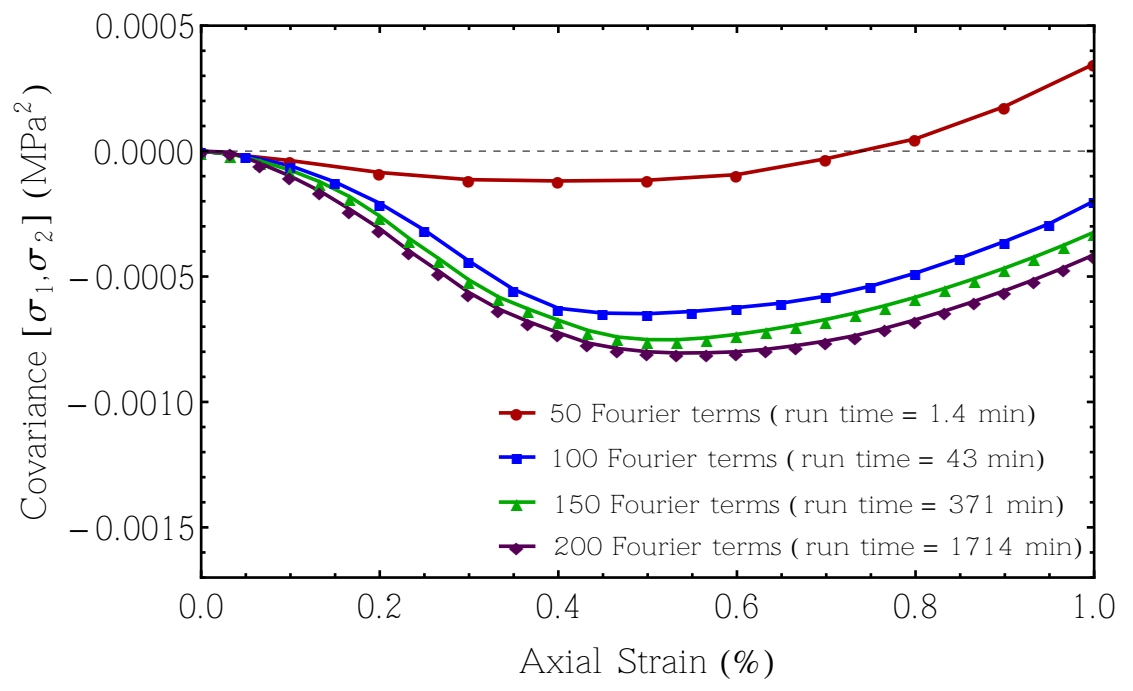

Figure 4.16: Effect of the number of Fourier terms on the simulated evolutionary covariance between the axial stress and radial stress with the axial strain for an uncertain von Mises elastic-perfectly plastic soil under an unconsolidated undrained triaxial compression test. 


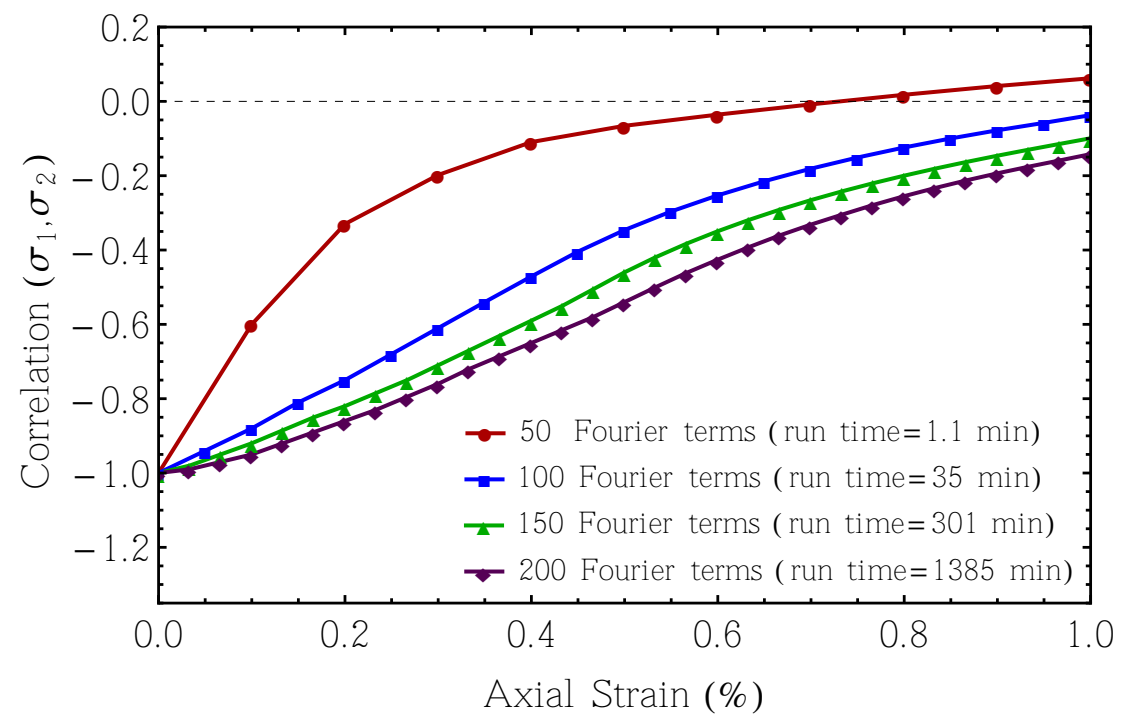

Figure 4.17: Effect of the number of Fourier terms on the simulated evolutionary correlation coefficient of the axial stress and radial stress with the axial strain for an uncertain von Mises elastic-perfectly plastic soil under an unconsolidated undrained triaxial compression test.

In this context, it is important to emphasize that as mentioned in Section 3.3.4, the presented hybrid spectral-finite difference algorithm is conditionally stable - the product of the number of Fourier terms and (pseudo) time step-size ( $\Delta t$ in Eqs. (3.139) and (3.154)) should be less than 2. Based on the simulations performed in this study, a value between 0.2 and 2 is recommended for optimum efficiency and stability.

\subsection{Simulation of Uncertain Cyclic Constitutive Behaviors of Soils}

In this section, the developed algorithms for solving the univariate and bivariate FPK PDEs with both space- and time-dependent coefficients that govern the probabilistic 
constitutive behaviors of elastic-plastic soils are used to simulate the uncertain cyclic constitutive responses of von Mises elastic-perfectly plastic soils under the unconfined compression test and the unconsolidated undrained triaxial compression test. Note that all the results presented below are processed through a Kaiser window to minimize spurious oscillations in the solutions.

\subsubsection{Unconfined Compression Simulation}

To simulate the probabilistic constitutive responses of cyclic von Mises elastic-perfectly plastic soils under unconfined compression test, one hysteresis loop which consists of three branches (loading, unloading and reloading) is simulated. The loading branch is similar to the monotonic simulation and can be found up to $1 \%$ strain by solving Eq. (4.22) with the same coefficients as the monotonic simulation. Similarly, the unloading branch can be obtained up to $-1 \%$ strain by solving Eq. (4.22), however, in computing $N_{(1)}^{\sigma^{e q} v M}(\sigma)$ and $N_{(2)}^{\sigma^{e q}}$, $\dot{\epsilon}$ should be replace by $-\dot{\epsilon}$. Moreover, the $\sigma$-dependent probabilities appearing in Eq. (3.139) should be computed as follows:

$$
\begin{aligned}
\operatorname{Pr}\left[\sigma_{y} \leq \sigma\right] & =C D F\left[\text { Normal Distribution }\left[\left\langle\sigma_{y}\right\rangle, \sqrt{\operatorname{Var}\left[\sigma_{y}\right]}\right],-\sigma\right] \\
& =\frac{1}{2}\left[1+\operatorname{Erf}\left(\frac{-\sigma-\left\langle\sigma_{y}\right\rangle}{\sqrt{2 \operatorname{Var}\left[\sigma_{y}\right]}}\right)\right]
\end{aligned}
$$

Finally, the reloading branch is obtained up to $1 \%$ strain by solving Eq. (4.22) with the same coefficients as the loading simulation. It should be mentioned that the initial condition of the unloading branch is the solution of the loading branch at the 
$1 \%$ strain, and the initial condition of the reloading branch is the solution of unloading branch at $-1 \%$ strain. Note that, all the parameters are assumed to be the same as the parameters used for the monotonic simulation.

Simulation results are shown in Figure 4.18 in terms of the probability density function (PDF) of the stresses at the end of loading, unloading and reloading. It is important to mention that the PDF of the response at each strain level contains all the information that one may need in any engineering problem. For instance, the statistical moments, such as the evolutionary mean and standard deviation of the stress, can be obtained by post-processing the evolutionary PDF of the stress. The results are illustrated in Figures 4.19 and 4.20 .

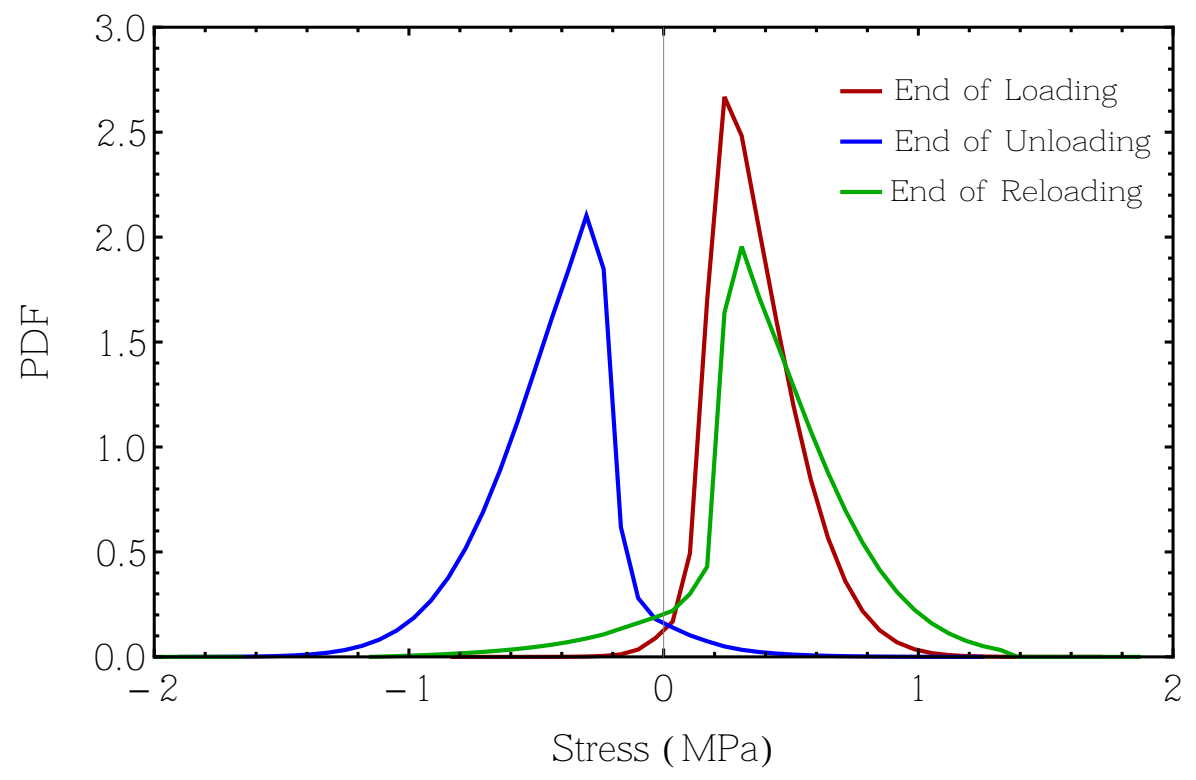

Figure 4.18: Probability density function (PDF) of the stress for an uncertain von Mises cyclic elastic-perfectly plastic soil under an unconfined compression test. 
As can be seen in Figure 4.19, the mean stress is nonlinear even at very small strain. This is due to the uncertainty in the yield strength which means that there is always a chance that soil plastifies at a very small strain. Moreover, it is possible that soil remains elastic at strains past the yield point. Therefore, the mean solution can be explained as the ensemble average of all the possibilities. This agrees well with the soil behavior that is usually observed in any laboratory experiment. In general, within a laboratory specimen (considered as representative volume element [19]) each particle contacts has different yield strength and the observed soil behavior in the experiment is the ensemble average (mean) behavior of all the particle contacts. This figure also shows that by considering the uncertainties in material properties, the hardening and Bauschinger effect can also be captured even with a simple von Mises elastic-perfectly plastic (two-parameters) model. On the other hand, Figure 4.20 shows the evolutionary standard deviation behavior obtained by post-processing the evolutionary PDF of the stress. It is important to note that the standard deviation behavior depends on the soil parameters, and by using a different values of $C O V$ for elastic modulus $(E)$ or yield strength $\left(\sigma_{y}\right)$, a totally different behavior may be observed. In general, when the material is elastic, both the uncertainties in elastic modulus $(E)$ and yield strength $\left(\sigma_{y}\right)$ are governing. However, as soil becomes elastic-plastic, the influence of uncertainty in elastic modulus $(E)$ decreases, and the uncertainty in yield strength is governing. This figure also shows that the value of the standard deviation is relatively large. This can be due to the modeling uncertainty which may be reduced by using more advanced (isotropic/kinematic hardening) plasticity models. 


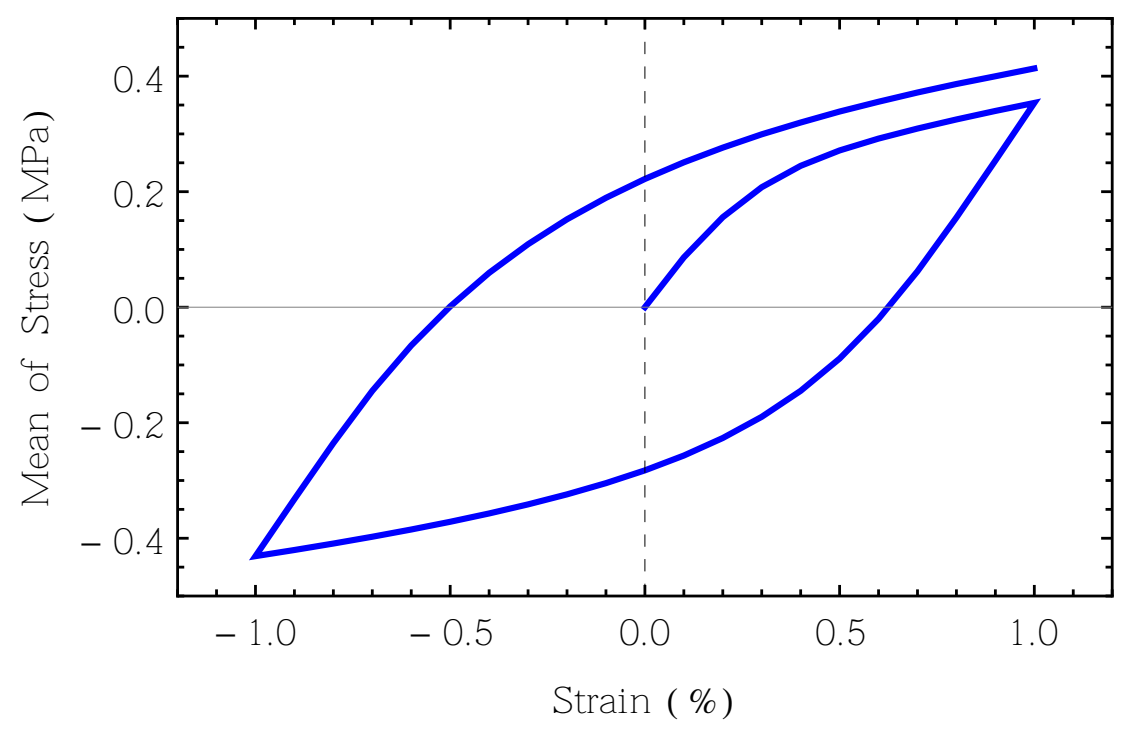

Figure 4.19: Simulated probabilistic cyclic elastic-perfectly plastic behavior in terms of the evolutionary mean behavior.

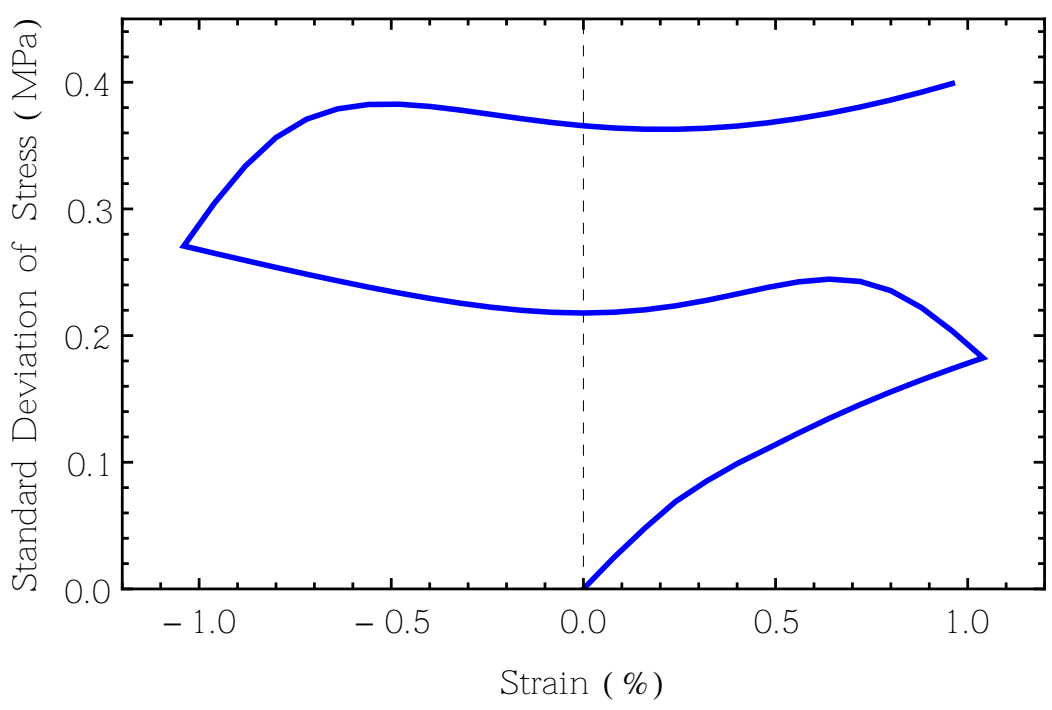

Figure 4.20: Simulated probabilistic cyclic elastic-perfectly plastic behavior in terms of the evolutionary standard deviation behavior. 


\subsubsection{Unconsolidated Undrained Triaxial Compression Simulation}

One of the most commonly used tests for measurement of dynamic soil properties is the cyclic triaxial test. In this section, the FPK approach is employed to simulate the probabilistic constitutive responses of cyclic von Mises elastic-perfectly plastic soils under unconsolidated undrained triaxial compression test.

To this end, one hysteresis loop which consists of three branches (loading, unloading and reloading) is simulated. The loading branch is similar to the monotonic simulation and can be found up to $1 \%$ strain by solving Eqs. (4.24). Note that, in the cyclic simulation, $\operatorname{Pr}\left[\sigma_{y} \leq \sqrt{3 J_{2}}\right]$ in Eq. (3.154) should be replaced by $\operatorname{Pr}\left[\sigma_{y}^{2} \leq 3 J_{2}\right]$. Therefore, by using Eq. (3.145) $\left(\sigma_{y}^{2}=3 J_{2}=\left(\sigma_{1}-\sigma_{2}\right)^{2}\right)$ the $J_{2}$-dependent probabilities appearing in Eq. (3.154) were computed as:

$$
\begin{array}{r}
\operatorname{Pr}\left[\sigma_{y}^{2} \leq 3 J_{2}\right]=C D F\left[\text { Weibull Distribution }[\theta, \kappa],\left(\sigma_{1}-\sigma_{2}\right)^{2}\right] \\
=\left\{\begin{array}{lr}
1-e^{-\left(\frac{\left(\sigma_{1}-\sigma_{2}\right)^{2}}{\theta}\right)^{\kappa}} & \text { when }\left(\sigma_{1}-\sigma_{2}\right) \geq 0 \\
0 & \text { when }\left(\sigma_{1}-\sigma_{2}\right)<0
\end{array}\right.
\end{array}
$$

Note that, $\theta$ and $\kappa$ are the parameters of the Weibull random variable $\sigma_{y}^{2}$.

Similarly, the unloading branch can be obtained up to $-1 \%$ strain by solving Eq. (4.22), however, in computing $N_{(1)_{r}}^{\sigma^{e q M}}(\sigma)$ and $N_{(2)_{r}}^{\sigma^{e q M}}, \dot{\epsilon}_{1}$ should be replace by $-\dot{\epsilon}_{1}$. Moreover, the $J_{2}$-dependent probabilities appearing in Eq. (3.154) were computed as: 


$$
\begin{array}{r}
\operatorname{Pr}\left[\sigma_{y}^{2} \leq 3 J_{2}\right]=C D F\left[\text { Weibull Distribution }[\theta, \kappa],\left(\sigma_{1}-\sigma_{2}\right)^{2}\right] \\
=\left\{\begin{array}{lr}
1-e^{-\left(\frac{\left(\sigma_{1}-\sigma_{2}\right)^{2}}{\theta}\right)^{\kappa}} & \text { when }\left(-\sigma_{1}+\sigma_{2}\right) \geq 0 \\
0 & \text { when }\left(-\sigma_{1}+\sigma_{2}\right)<0
\end{array}\right.
\end{array}
$$

Finally, the reloading branch is obtained up to $1 \%$ strain by solving Eqs. (4.22) with the same coefficients as the loading simulation. It should be mentioned that the initial condition of the unloading branch is the solution of the loading branch at the $1 \%$ strain, and the initial condition of the reloading branch is the solution of unloading branch at $-1 \%$ strain. Note that, all the parameters are assumed to be the same as the parameters used for the monotonic simulation.

The simulation result is obtained in terms of the evolutionary joint PDF of the axial and radial stresses. The marginal PDFs of the axial and radial stresses can be computed by using the standard integration techniques. Figures 4.21 and 4.22 show the evolutionary marginal PDFs of the axial and radial stresses, which include all the information on the statistical moments of the stresses, at the end of loading, unloading and reloading.

Typical cyclic undrained responses [8] are also obtained in terms of the evolutionary mean and standard deviation of deviatoric stress, $q=\sigma_{1}-\sigma_{2}$, with axial strain $\left(\epsilon_{a}\right)$, and deviatoric stress $(q)$ with mean effective stress, $p^{\prime}=\frac{1}{3}\left(\sigma_{1}+2 \sigma_{2}\right)$, by post-processing the evolutionary joint PDF of the stresses (Figures 4.23 to 4.25). 


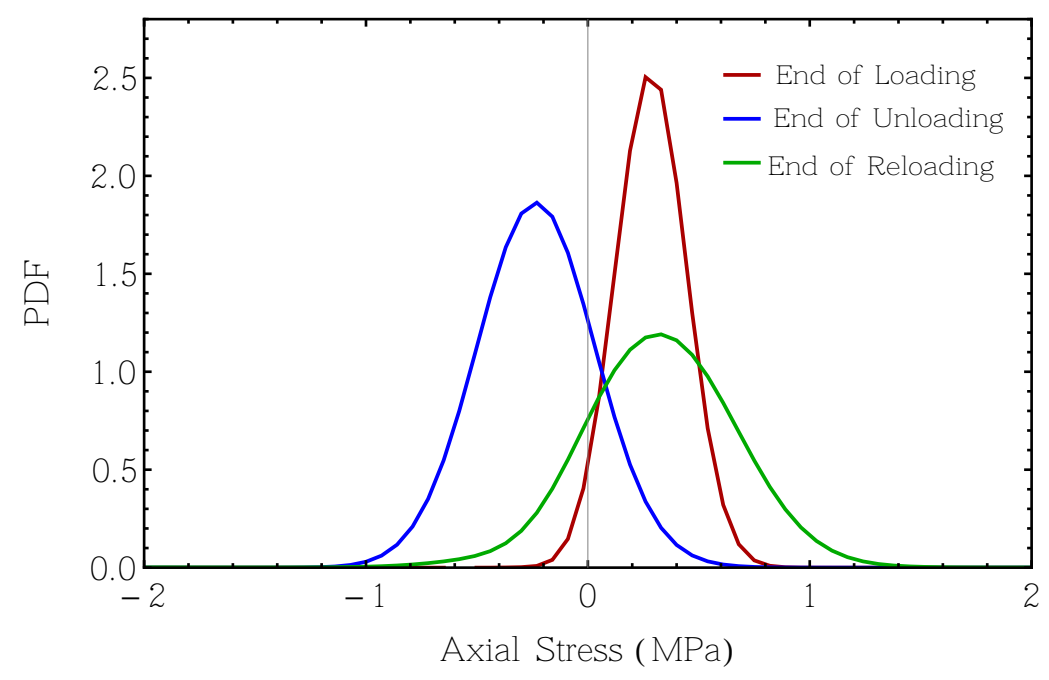

Figure 4.21: Probability density function (PDF) of the axial stress for an uncertain von Mises cyclic elastic-perfectly plastic soil under an unconfined compression test.

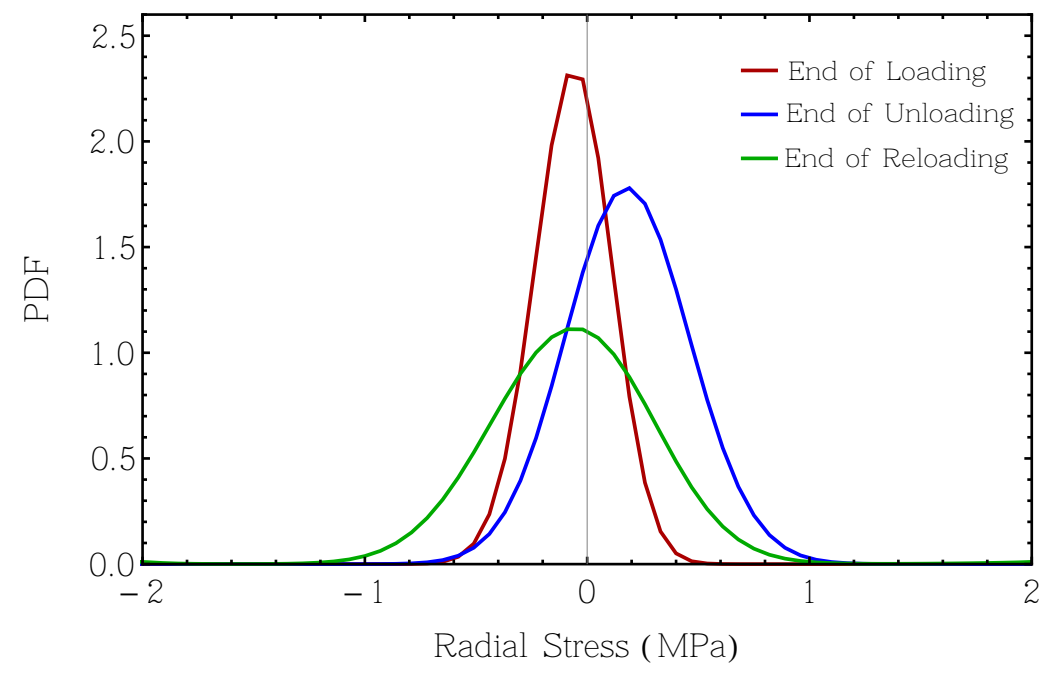

Figure 4.22: Probability density function (PDF) of the radial stress for an uncertain von Mises cyclic elastic-perfectly plastic soil under an unconfined compression test.

As can be seen in Figure 4.23, the mean deviator stress is nonlinear from the beginning. This shows that by considering the uncertainty in material properties, a 
realistic cyclic constitutive behavior which captures the hardening and Bauschinger effect can be obtained even with a simple elastic-perfectly plastic (two-parameters) model. This is especially important because in geotechnical engineering practice, advanced laboratory tests are rarely performed.

Figure 4.24 shows the evolutionary standard deviation behavior obtained by post-processing the evolutionary joint PDF of the stresses. As can be seen in this figure, the standard deviation always increases. This shows that as material plastifies, the two-parameter von Mises elastic-perfectly plastic becomes less and less accurate. Therefore, in order to get more accurate results, more advanced constitutive (isotropic/kinematic hardening) models are required. Note that this behavior is not generic, and it depends on the material parameters.

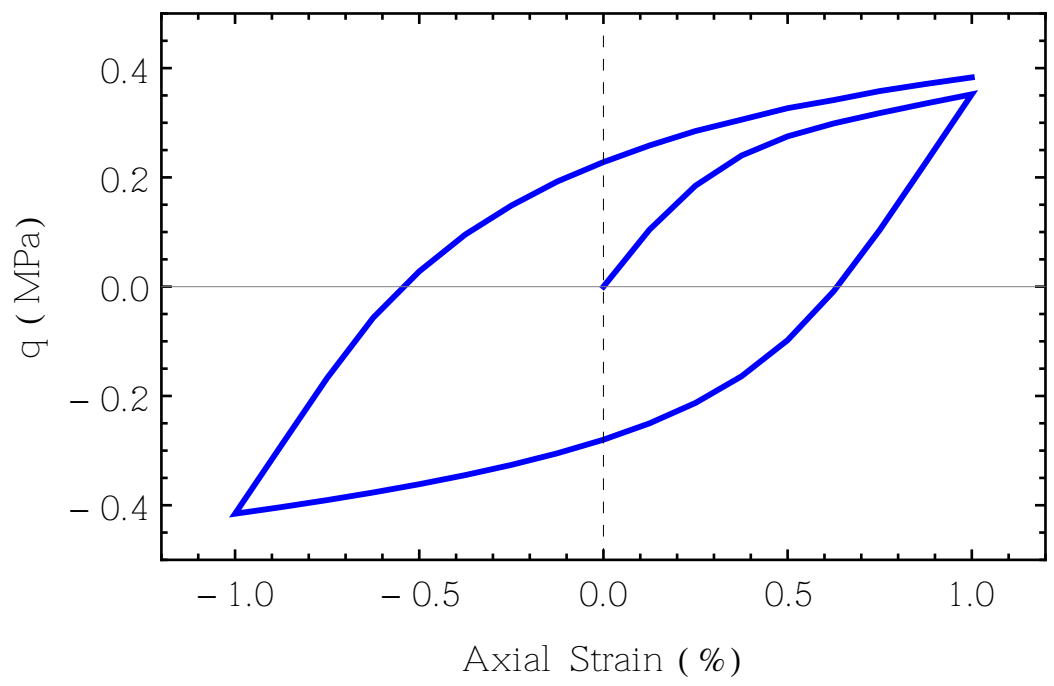

Figure 4.23: Simulated probabilistic cyclic elastic-perfectly plastic behavior in terms of the evolutionary mean of deviatoric stress with axial strain. 


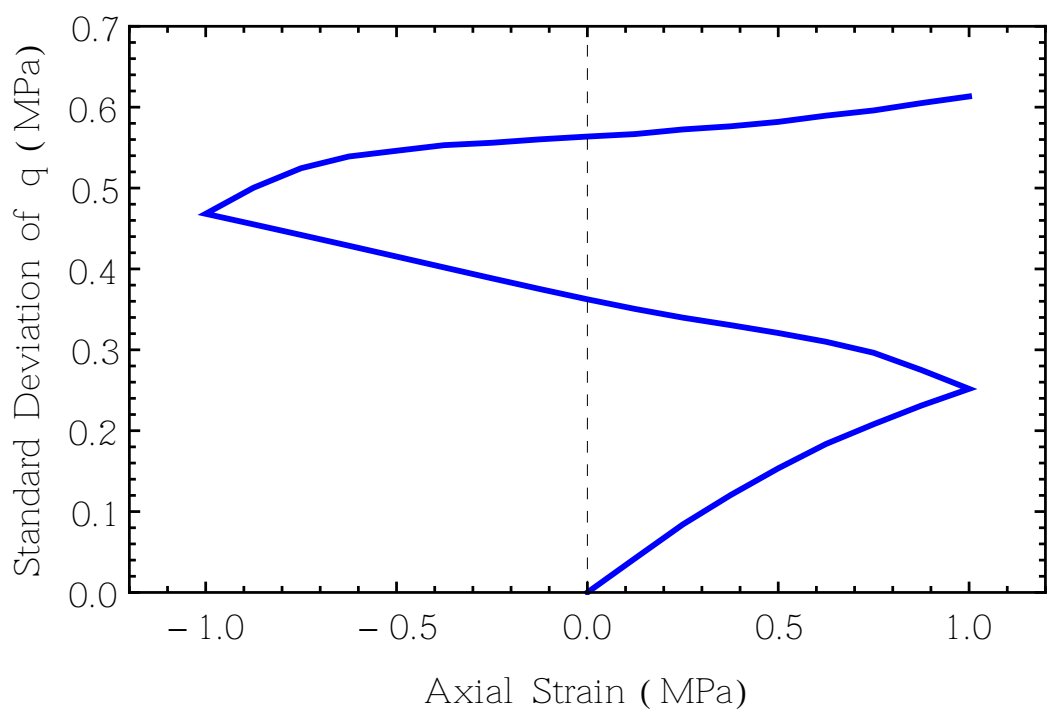

Figure 4.24: Simulated probabilistic cyclic elastic-perfectly plastic behavior in terms of the evolutionary standard deviation of deviatoric stress with axial strain.

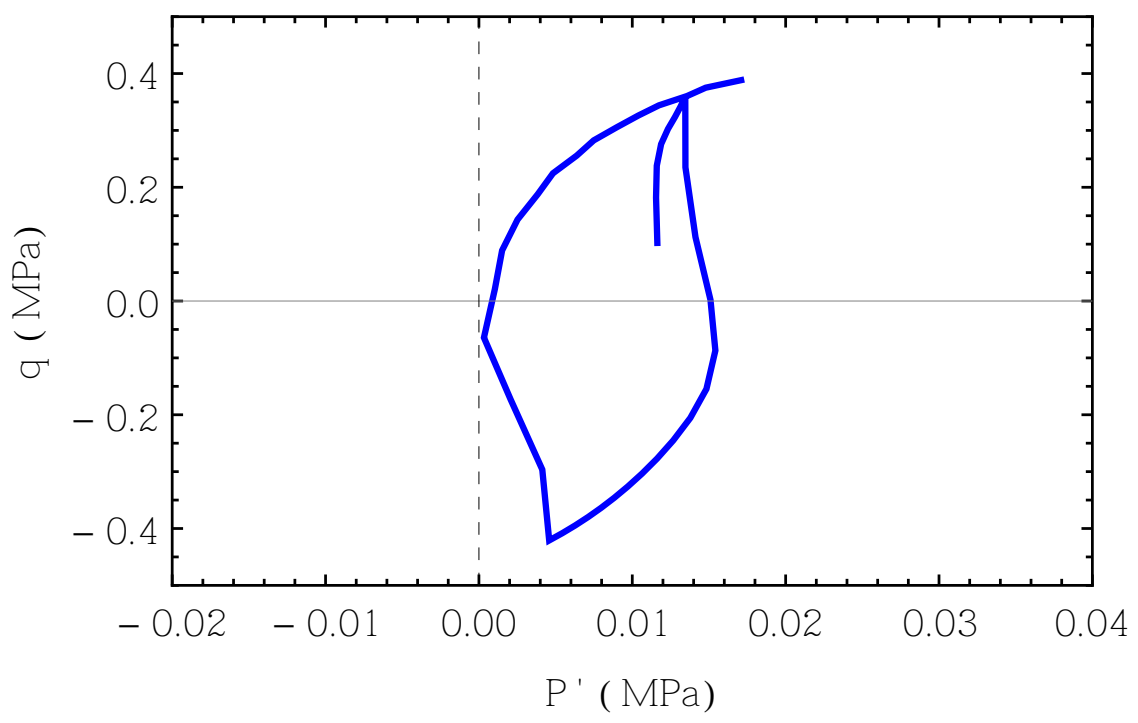

Figure 4.25: Simulated probabilistic cyclic elastic-perfectly plastic behavior in terms of the evolutionary mean of deviatoric stress with evolutionary mean effective stress. 


\section{CHAPTER V}

\section{CONCLUSIONS}

\subsection{Summary and Conclusions}

In this dissertation the existing one dimensional FPK framework for probabilistic constitutive modeling of soils was extended to multi-dimension. To this end, a set of numerical algorithms were first developed for efficient solutions of the FPK PDEs in probabilistic elasto-plasticity. For the PDEs with space-independent but timedependent coefficients that govern the constitutive behaviors of uncertain linear elastic solids, a semi-analytical algorithm based on the Fourier spectral method was proposed. On the other hand, for the PDEs with both space- and time-dependent coefficients that govern the constitutive behaviors of uncertain elastic-plastic solids, a hybrid Fourier spectral-finite difference algorithm was suggested. The developed algorithms were then used to probabilistically simulate the uniaxial and multiaxial, monotonic and cyclic constitutive behaviors of uncertain elasto-plastic soils. In particular, two common laboratory constitutive experiments in geotechnical engineering, namely, the unconfined compression test (a uniaxial problem) and the unconsolidated undrained triaxial compression test (a multiaxial problem) were considered. In the following, the main findings of this dissertation are enumerated: 
1. The accuracy of the developed algorithms was tested on both univariate and multivariate equations. It was observed that, even though, the computational costs increased with the increase in the number of Fourier terms, a small number of Fourier terms were enough to obtain reasonable accuracies.

2. The efficiency of the developed algorithms was also quantified in terms of the run time. The results showed that the developed algorithms were computationally more efficient than the traditional finite difference technique.

3. The evolutionary mean and standard deviation of the stress-strain behaviors were obtained by post-processing the results of the simulations. The evolutionary mean behavior could be explained as the ensemble average of behaviors of all the soil particles in a RVE (soil specimen). On the other hand, the evolutionary standard deviation behavior may be used as an indicator of the dispersion of the response around the mean. The region between the mean and mean \pm standard deviation, which contained the most probable values of the response, was also obtained.

4. The elastic monotonic unconsolidated undrained triaxial compression simulation results showed that the slopes of $\left\langle\mathrm{q}>-\epsilon_{a},\left\langle\mathrm{q}>-\epsilon_{q}\right.\right.$, and $\langle\mathrm{u}\rangle-\epsilon_{a}$ agreed with the classical soil mechanics theory. This indicated that for the linear elastic behavior, the mean response matched with the deterministic solution. However, by performing a probabilistic simulation, mean \pm standard deviation of the solution also could be obtained which illustrated that depending upon the 
uncertainties in the soil properties and interaction between them, there would be a band around the mean response that contained the most probable values of the response.

5. The elastic-perfectly plastic monotonic and cyclic unconsolidated undrained triaxial compression simulation results indicated that, even with the elasticperfectly plastic (i.e, bi-linear) model, due to uncertainty in yielding, the probabilistic response was smooth and nonlinear from the beginning. This response is very realistic and it means that depending upon the uncertainty in yield strength, there is always a possibility that the soil becomes elastic-plastic from the very beginning of loading, which agrees well with the fact that within a RVE of a spatially non-uniform material like soils some particle-to-particle slips may occur earlier than others.

6. It was shown that explicit treatment of soil uncertainties not only allows for quantification of our confidence in our predictions, but also modeling some of the important aspects of soil behaviors-for example, hardening and Bauschinger effect- even with the simplest elastic-perfectly plastic von Mises (two-parameters) model. This is particularly significant since in geotechnical engineering practice, advanced laboratory tests are rarely performed. 


\subsection{Future work}

During the course of this research, five main related topics emerged as future research directions:

1. In this dissertation, the FPK approach is used to probabilistically simulate the elastic-perfectly plastic monotonic and cyclic undrained triaxial behavior of soils. It will be really interesting to investigate the uncertain elastic-perfectly plastic monotonic and cyclic triaxial behavior of soils when drainage can occur freely from the soil sample. This simulation is similar to the undrained simulation, however, the volumetric strain is changing. Therefore, the Poisson's ratio needs to be updated during the simulation.

2. In this dissertation, the probabilistic elastic-perfectly plastic triaxial behavior of soils was simulated by solving only one FPK PDE and the internal variable FPK PDE did not exist. Using more advanced constitutive (isotropic/kinematic hardening) models, potentially can reduce modeling uncertainty. Therefore, it will be interesting to use advanced constitutive models for simulating the uncertain soil behavior which can be done using the developed multi-dimensional framework. In order to do this, the two coupled FPK PDEs discussed in chapter 2 should be solved simultaneously for the first step. Then the obtained information should be used to update the coefficients of the first FPK PDE, and the two coupled FPK PDEs should be solved again. This process should be repeated for all the steps. 
3. In this study, the general FPK equations were specialized to uniaxial stress space and to two-dimensional principal stress space. However, in the general multidimensional formulation, the stress tensor contains six independent components. The 6-D implementation of the presented general formulation will have much wider applications in the field of geotechnical engineering.

4. In this research, the multi-dimensional probabilistic constitutive simulation of soils was presented. By integrating the developed multi-dimensional framework with the spectral approach, a stochastic finite element (SFE) framework may be developed which can be used for simulating the real world problems. 


\section{BIBLIOGRAPHY}

[1] Anders, M., AND Hori, M. Stochastic finite element method for elasto-plastic body. International Journal for Numerical Methods in Engineering 46, 11 (1999), 1897-1916.

[2] Anders, M., AND Hori, M. Three-dimensional stochastic finite element method for elasto-plastic bodies. International Journal for Numerical Methods in Engineering 51, 4 (June 2001), 449-478.

[3] A.V. Oppenheim, R. S. Discrete-time signal processing. Pearson Education, New Jersey.

[4] Baecher, G. B., And Christian, J. T. Reliability and Statistics in Geotechnical Engineering, second ed. Wiley, West Sussex PO19 8SQ, England, 2003.

[5] Boyd, J. P. Chebyshev and Fourier Spectral Methods, second ed. Courier Dover, New York, 1941.

[6] Chen, W. F., And Han, D. J. Plasticity for Structural Engineers. SpringerVerlag, 1988.

[7] Clenshaw, C. W. A note on the summation of chebyshev series. Mathematics of Computation 9 (1955), 118-120.

[8] Dafalias, Y. F., And Manzari, M. T. Simple plasticity sand model accounting for fabric change effects. ASCE Journal of Engineering Mechanics 130, 6 (June 2004), 622-634.

[9] Das, B. M. Soil Mechanics Laboratory Manual, eighth ed. Oxford University Press, New York, NY, 2013.

[10] De Lima, B. S. L. P., Teixeira, E. C., and Ebecken, N. F. F. Probabilistic and possibilistic methods for the elastoplastic analysis of soils. Advances in Engineering Software 132 (2001), 569-585. 
[11] DeGroot, D. J., And Baecher, G. B. Estimating autocovariance of insitu soil properties. Journal of Geotechnical Engineering 119, 1 (January 1993), $147-166$.

[12] Fenton, G. A. Estimation of stochastic soil models. Journal of Geotechnical and Geoenvironmental Engineering, ASCE 125, 6 (June 1999), 470-485.

[13] Fenton, G. A. Random field modeling of CPT data. Journal of Geotechnical and Geoenvironmental Engineering, ASCE 125, 6 (June 1999), 486-498.

[14] Gardiner, C. W. Handbook of Stochastic Methods for Physics, Chemistry and the Natural Science, third ed. Springer:Complexity. Springer-Verlag, Berlin Heidelberg, 2004.

[15] Ghanem, R. G., and Spanos, P. D. Stochastic Finite Elements: A Spectral Approach. Springer-Verlag, 1991. (Reissued by Dover Publications, 2003).

[16] Griffiths, D. V., Fenton, G. A., and Manoharan, N. Bearing capacity of rough rigid strip footing on cohesive soil: Probabilistic study. Journal of Geotechnical and Geoenvironmental Engineering, ASCE 128, 9 (2002), 743-755.

[17] Hammitт, G. M. Statistical analysis of data from a comparative laboratory test program sponsored by ACITL. U.S. Army Waterways Experiment Station, Vicksburg, MS, 1966.

[18] Hara, A., Ohta, T., Niwa, M., Tanaka, S., and Banno, T. Shear modulus and shear strength of cohesive soils. Soils and Foundations 14, 3 (1974), $1-12$.

[19] Hashin, Z. Analysis of composite materials. Journal of Applied Mechanics 50 (1983), 481-501.

[20] He, Q., And Jian, D. An explicit time-domain finite-element method that is unconditionally stable. In Proceedings of the 2011 IEEE International Symposium on Antennas and Propagation (2011), Antennas and Propagation Society, IEEE, pp. 2976-2979.

[21] Hindmarsh, A. C., Brown, P. N., Grant, K. E., Lee, S. L., SerBan, R., Shumaker, D. E., and Woodward, C. S. SUNDIALS: SUite of Nonlinear and DIfferential/ALgebraic equation Solvers. ACM Transactions on Mathematical Software 31, 3 (2005), 363-396. 
[22] H.O. Kreiss, J. O. Methods for the approximate solution of time-dependent problems. Global Atmospheric Research Programme Publications Series 10 (1973).

[23] Jeremić, B., And Sett, K. On probabilistic yielding of materials. Communications in Numerical Methods in Engineering 25, 3 (2009), 291-300.

[24] Jeremić, B., Sett, K., And Kavvas, M. L. Probabilistic elasto-plasticity: Formulation in 1-D. Acta Geotechnica 2, 3 (September 2007), 197-210.

[25] JiAO, D., AND Jin, J. M. A general approach for the stability analysis of timedomain finite element method. IEEE Transactions on Antennas and Propagation 50, 11 (2002), 1624-1632.

[26] Karhunen, K. Über lineare methoden in der wahrscheinlichkeitsrechnung. Ann. Acad. Sci. Fennicae. Ser. A. I. Math.-Phys., 37 (1947), 1-79.

[27] Kavvas, M. L. Nonlinear hydrologic processes: Conservation equations for determining their means and probability distributions. Journal of Hydrologic Engineering 8, 2 (March 2003), 44-53.

[28] Kleiber, M., And Hien, T. D. The Stochastic Finite Element Method: Basic Perturbation Technique and Computer Implementation. John Wiley \& Sons, Baffins Lane, Chichester, West Sussex PO19 1UD , England, 1992.

[29] Koutsourelakis, S., Prevost, J. H., and Deodatis, G. Risk assesment of an interacting structure-soil system due to liquefaction. Earthquake Engineering and Structural Dynamics 31 (2002), 851-879.

[30] Lacasse, S., And Nadim, F. Uncertainties in characterizing soil properties. In Uncertainty in Geologic Environment: From Theory to Practice, Proceedings of Uncertainty '96, July 31-August 3, 1996, Madison, Wisconsin (1996), C. D. Shackelford and P. P. Nelson, Eds., vol. 1 of Geotechnical Special Publication No. 58, ASCE, New York, pp. 49-75.

[31] Lanczos, C. Applied Analysis. Prentice-Hall, New Jersey, 1956.

[32] LoÈve, M. Fonctions aléatoires du second ordre. Supplément to P. Lévy, Processus Stochastiques et Mouvement Brownien, Gauthier-Villars, Paris, 1948.

[33] Marosi, K. T., and Hiltunen, D. R. Characterization of spectral analysis of surface waves shear wave velocity measurement uncertainty. Journal of Geotechnical and Geoenvironmental Engineering, ASCE 130, 10 (October 2004), 1034-1041. 
[34] Mayne, P. Stress-strain-strength-flow parameters from enhanced in-situ tests. In Proceedings of the International Conference on In-Situ Measurement of Soil Properties $\&$ Case Histories, Bali, Indonesia, May 21-24, 2001 (2001), pp. $27-$ 48.

[35] Metropolis, N., And Ulam, S. The Monte Carlo method. Journal of the American Statistical Association 44, 247 (1949), 335-341.

[36] Montgomery, D. C., And Runger, G. C. Applied Statistics and Probability for Engineers, third ed. John Wiley \& Sons, 605 Third Avenue, New York, NY 10158, 2003.

[37] Paice, G. M., Griffiths, D. V., and Fenton, G. A. Finite element modeling of settlement on spatially random soil. Journal of Geotechnical Engineering 122, 9 (1996), 777-779.

[38] Phoon, K.-K., and Kulhawy, F. H. Characterization of geotechnical variability. Canadian Geotechnical Journal 36, 4 (1999), 612-624.

[39] Phoon, K.-K., and Kulhawy, F. H. Evaluation of geotechnical property variability. Canadian Geotechnical Journal 36, 4 (1999), 625-639.

[40] Popescu, R., Prevost, J. H., and Deodatis, G. Effects of spatial variability on soil liquefaction: Some design recommendations. Geotechnique 47, 5 (1997), 1019-1036.

[41] S.A. Orszag, M. I. Numerical simulations of incompressible flow. Fluid Mechanics 6 (1974), 281-318.

[42] Schüeller, G. I. A state-of-the-art report on computational stochastic mechanics. Probabilistic Engineering Mechanics 12, 4 (1997), 197-321.

[43] Sett, K., And Jeremić, B. Probabilistic yielding and cyclic behavior of geomaterials. International Journal for Numerical and Analytical Methods in Geomechanics 34, 15 (2010), 1541-1559.

[44] Sett, K., Jeremić, B., And Kavvas, M. L. Probabilistic elasto-plasticity: Solution and verification in 1-D. Acta Geotechnica 2, 3 (September 2007), 211220.

[45] Sett, K., Jeremić, B., And Kavvas, M. L. The role of nonlinear hardening/softening in probabilistic elasto-plasticity. International Journal for $\mathrm{Nu}$ merical and Analytical Methods in Geomechanics 31, 7 (June 2007), 953-975. 
[46] Sett, K., Jeremić, B., and Kavvas, M. L. Stochastic elastic-plastic finite elements. Computer Methods in Applied Mechanics and Engineering 200, 9-12 (February 2011), 997-1007.

[47] Sudret, B., And Der Kiureghian, A. Stochastic finite element methods and reliability: A state of the art report. Technical Report UCB/SEMM-2000/08, University of California, Berkeley, 2000.

[48] VOn Mises, L. Mechanik der festen koerper in plastisch deformablem zustand. Goettinger Nachr. Math. Phys (1913), 582-592.

[49] Wiener, N. The homogeneous chaos. American Journal of Mathematics 60, 4 (1938), 897-936.

[50] Wood, D. M. Soil Behaviour and Critical State Soil Mechanics. Cambridge University Press, Cambridge CB2 8RU, UK, 1990. ISBN : 978-0-521-33782-3.

[51] Xıu, D. Numerical Methods for Stochastic Computations: A Spectral Method Approach. Princeton University Press, New Jersey, 2010.

[52] Zienkiewicz, O. C., And Taylor, R. L. The Finite Element Method Volume 1, the Basis, fifth ed. Butterwort-Heinemann, Oxford, 2000. 